ESAIM: M2AN 50 (2016) 1523-1560

DOI: $10.1051 / \mathrm{m} 2 \mathrm{an} / 2015092$
ESAIM: Mathematical Modelling and Numerical Analysis

www.esaim-m2an.org

\title{
ENERGY STABLE AND CONVERGENT FINITE ELEMENT SCHEMES FOR THE MODIFIED PHASE FIELD CRYSTAL EQUATION ${ }^{*, * *, * * *}$
}

\author{
Maurizio Grasselli ${ }^{1}$ and Morgan Pierre ${ }^{2}$
}

\begin{abstract}
We propose a space semi-discrete and a fully discrete finite element scheme for the modified phase field crystal equation (MPFC). The space discretization is based on a splitting method and on a Galerkin approximation in $H^{1}$ for the phase function. This formulation includes the classical continuous finite elements. The time discretization is a second-order scheme which has been introduced by Gomez and Hughes for the Cahn-Hilliard equation. The fully discrete scheme is shown to be unconditionally energy stable and uniquely solvable for small time steps, with a smallness condition independent of the space step. Using energy estimates, we prove that in both cases, the discrete solution converges to the unique energy solution of the MPFC equation as the discretization parameters tend to 0 . This is the first proof of convergence for the scheme of Gomez and Hughes, which has been shown to be unconditionally energy stable for several Cahn-Hilliard related equations. Using a Eojasiewicz inequality, we also establish that the discrete solution tends to a stationary solution as time goes to infinity. Numerical simulations with continuous piecewise linear $\left(P^{1}\right)$ finite elements illustrate the theoretical results.
\end{abstract}

Mathematics Subject Classification. 65M60, 65P40, 74N05, 82C26.

Received February 24, 2015. Revised October 19, 2015. Accepted November 24, 2015.

\section{INTRODUCTION}

In this paper, we analyze finite element discretizations of the modified phase field crystal (MPFC) equation

$$
\beta u_{t t}+u_{t}=\Delta\left[\Delta^{2} u+2 \Delta u+f(u)\right], \quad \text { in } \Omega \times(0,+\infty),
$$

Keywords and phrases. Finite elements, second-order schemes, gradient-like systems, Łojasiewicz inequality.

* The first author thanks the Laboratoire de Mathématiques et Applications for the kind hospitality and the Université de Poitiers which supported his stay in May 2014.

** The first author is member of the Gruppo Nazionale per l'Analisi Matematica, la Probabilità e le loro Applicazioni (GNAMPA) of the Istituto Nazionale di Alta Matematica (INdAM).

*** The second author thanks the Dipartimento di Matematica of the Politecnico di Milano for the invitation in February 2014.

1 Dipartimento di Matematica, Politecnico di Milano, Via E. Bonardi 9, 20133 Milano, Italy. maurizio.grasselli@polimi.it

2 Laboratoire de Mathématiques et Applications UMR CNRS 7348, Université de Poitiers, Téléport 2 - BP 30179 , Boulevard Marie et Pierre Curie, 86962 Futuroscope Chasseneuil, France. Morgan.Pierre@math.univ-poitiers.fr 
with periodic boundary conditions on the $d$-parallelepiped $\Omega=\Pi_{k=1}^{d}\left(0, L_{k}\right)\left(L_{k}>0\right.$ for $\left.k=1, \ldots, d\right)$ in space dimension $d=1,2$ or 3. Equation (1.1) is endowed with initial conditions

$$
\left.u\right|_{t=0}=u_{0}(x),\left.\quad u_{t}\right|_{t=0}=v_{0}(x), \quad x \in \Omega .
$$

The unknown function $u$ is the phase function and $\beta>0$ is a relaxation parameter. The nonlinearity $f$ is the derivative of a polynomial potential $F$ (see (2.1) and (2.2) for a precise definition). A relevant example in applications is given by:

$$
f(s)=s^{3}+(1-\varepsilon) s,
$$

where $\varepsilon \in \mathbb{R}$ is constant.

When $\beta=0$, equation (1.1) is known as the phase field crystal (PFC) equation: it has been employed to model and simulate the dynamics of crystalline materials, including crystal growth in a supercooled liquid, dendritic and eutectic solidification, epitaxial growth $[8,9,12,13,34,36]$. In the phase field approach, the number density of atoms is approximated by the phase function $u$. The parameter $\varepsilon$ in (1.3) is proportional to the undercooling i.e. $\varepsilon \sim T_{e}-T, T_{e}$ being the equilibrium temperature at which the phase transition occurs. The PFC equation is a gradient flow for the free energy

$$
E(u)=\int_{\Omega}\left(\frac{1}{2}|\Delta u|^{2}-|\nabla u|^{2}+F(u)\right) \mathrm{d} x .
$$

It preserves the total mass and can be viewed as an analog of the Swift-Hohenberg equation [40].

The MPFC equation (1.1) (with $\beta>0$ ) was recently proposed in [38] (cf. also [13,15,17,39]) in order to account for elastic interactions. Equations like (1.1) have also been derived in [16] to take large deviations from thermodynamic equilibrium into account. The MPFC equation is no longer a gradient flow for (1.4), but it is possible to associate to a solution $\left(u, u_{t}\right)$ a "pseudo-energy", obtained by adding to $E$ a kinetic energy term (see (2.7)). This leads in a natural way to the notion of energy solution introduced by Grasselli and Wu [23] for the MPFC equation, following a terminology used for the modified Cahn-Hilliard equation [25-27]. Existence of a unique energy solution was proved by Grasselli and $\mathrm{Wu}$ in [23] as well as the convergence of single trajectories to single stationary states. We point out that the proof of uniqueness is based on a nonstandard argument. The analysis of global dynamics (that is, existence of global and exponential attractors) for the MPFC equation was carried out in $[23,24]$.

From the numerical analysis point of view, the MPFC equation has been studied in $[4,5,18,41,42]$, while the literature on the PFC equation is more abundant (see, e.g., $[3,7,11,20,29,43])$. In $[4,5,42]$, the authors proposed unconditionally energy stable and unconditionally uniquely solvable finite difference schemes. The time discretization was based on a convex splitting of the pseudo-energy and was either first order or second order. A priori error estimates were proved assuming enough regularity on the solution. A time semi-discrete scheme was used in [41] to establish the existence of a weak solution and of a unique strong solution to the MPFC equation up to any positive final time $T>0$. In [18], an unconditionally energy stable finite element scheme was derived, but no proof of convergence was given.

The main purpose of this paper is to derive and analyze a second order (in time) fully discrete finite element scheme for the MPFC equation. For the space discretization we use a splitting approach which is well known in the context of phase field models $(c f ., e . g .,[10,21])$. The analysis is carried out for a Galerkin approximation in $H_{\text {per }}^{1}$ (see Sect. 2); it includes the classical continuous $\left(\mathrm{C}^{0}\right)$ finite elements, for instance the $P^{k}$ and $Q^{k}$ finite elements of any order $k$. In view of (1.4), a more natural choice could have been a Galerkin approximation in $H_{p e r}^{2}$, but this would require continuously differentiable $\left(C^{1}\right)$ finite elements. For the time discretization, we use a modified Crank-Nicolson scheme which was introduced by Gomez and Hughes for the Cahn-Hilliard equation [19], and which has been shown to be unconditionally energy stable for several gradient-like equations [18-20]. Their approach is based on a decomposition of the nonlinearity and it represents an interesting alternative to secant schemes ( $c f$. the discussion in [44]). 
We prove that our scheme is unconditionally energy stable, solvable for any time step, and uniquely solvable for small enough time steps, with a smallness assumption independent of the space step. Using the energy estimate, and assuming only some natural conditions on the initial conditions, we show that the solution of the fully discrete scheme converges to the energy solution of problem (1.1) and (1.2) as the time step $\tau$ and the space step $h$ tend to 0 . To our knowledge, this is the first proof of well-posedness and convergence for the scheme of Gomez and Hughes: for this purpose, we have introduced a decomposition of the linearity which is more specific than the one usually required (see assumption (H3) and Rem. 4.10). Finally, we prove that the discrete solution tends to a stationary solution as time goes to infinity. This last issue is not trivial since the set of steady states can be very complicated (see $[1,35]$ for an analysis of the one dimensional stationary problem).

For equations involving a second order time derivative such as (1.1), second-order time discretizations are very interesting because they do not regularize in finite time, unlike first order schemes: a fundamental property of the continuous model is therefore reproduced at the discrete level. In contrast with the second order two-step scheme in $[4,5]$, we loose the unconditional unique solvability, but one advantage is that our one-step scheme can be used with variable time steps. Moreover, we do not assume $\varepsilon<1$ in (1.3), and we do not have any restriction on the initial value of $u_{t}$. Our convergence result as $(h, \tau) \rightarrow(0,0)$ holds for any energy solution, and this can be considered as the minimal regularity requirement for the MPFC equation [23]. In contrast, standard error estimates would require much more regularity, and these are hard to obtain for this problem [23, 24].

Our proofs are crucially based on the energy estimates. In order to establish the convergence to equilibrium, we also use the gradient-like structure of the problem and a Łojasiewicz inequality [32], as in the continuous case [23]. Related results have been obtained for first order schemes applied to second-order gradient-like equations in $[2,22]$, but the case of the second-order scheme here is technically more involved. For completeness, we mention that convergence to equilibrium for second-order discretizations of gradient or gradient-like flows in infinite dimension is an open question (for the first-order case, see [6,33]).

The paper is structured as follows. In Section 2 we introduce the functional setting and we recall useful results concerning the continuous problem. In Section 3 we consider the space semi-discrete problem. We show its well-posedness and establish energy estimates which allow us to prove the convergence of the semi-discrete solution to the energy solution of the MPFC problem (1.1) and (1.2). This gives a framework for the fully discrete problem which is treated in Section 4. Section 5 is concerned with the convergence to equilibrium for the fully discrete problem. In Section 6, numerical simulations with piecewise linear $\left(P^{1}\right)$ finite elements in one and two space dimension illustrate the theoretical results.

\section{The CONTINUOUS PROBLEM}

\subsection{Notation and functional spaces}

For a real Banach space $V$ with dual $V^{\star}$, we indicate by $\langle\cdot, \cdot\rangle_{V^{\star}, V}$ the duality product between $V$ and $V^{\star}$. We denote by $H_{\text {per }}^{m}, m \in \mathbb{N}$, the space of functions that are in $H_{l o c}^{m}\left(\mathbb{R}^{d}\right)$ and periodic with period $\Omega$. For any $m \in \mathbb{N}, H_{p e r}^{m}$ is a Hilbert space for the scalar product

$$
((u, v))_{m}=\sum_{|\kappa| \leq m} \int_{\Omega} D^{\kappa} u(x) D^{\kappa} v(x) \mathrm{d} x
$$

( $\kappa$ being a multi-index) and its associated norm $\|u\|_{m}=((u, u))_{m}^{1 / 2}$.

For $m=0, H_{\text {per }}^{0}=L^{2}(\Omega)$, the inner product as well as the norm on $L^{2}(\Omega)$ are simply indicated by $(\cdot, \cdot)$ and $\|\cdot\|$, respectively. For sake of simplicity, we assume that $\int_{\Omega} 1 \mathrm{~d} x=|\Omega|=1$. The mean value of any function $u \in L^{2}(\Omega)$ is denoted by

$$
\langle u\rangle=\int_{\Omega} u \mathrm{~d} x
$$

and we set $\dot{u}=u-\langle u\rangle$. 
The dual space of $H_{p e r}^{m}$ is denoted by $H_{p e r}^{-m}$, and it is equipped with the operator norm given by:

$$
\|\mathcal{T}\|_{-m}=\sup _{\|u\|_{m}=1, u \in H_{p e r}^{m}}|\mathcal{T}(u)|
$$

For an operator $u \in H_{p e r}^{-m}$, we denote $\langle u\rangle=\langle u, 1\rangle_{H_{p e r}^{-m}, H_{p e r}^{m}}$ and we set $\dot{u}=u-\langle u\rangle$. We denote by $\dot{H}_{p e r}^{m}=\{u \in$ $\left.H_{\text {per }}^{m}:\langle u\rangle=0\right\}(m \in \mathbb{Z})$ the Sobolev spaces for functions with zero mean. We will frequently use the fact that $H_{\text {per }}^{m}$ is isomorphic to $\mathbb{R} \times \dot{H}_{\text {per }}^{m}(m \in \mathbb{Z})$ through the decomposition $u=\langle u\rangle+\dot{u}$.

Using the dense and continuous inclusions $H_{\text {per }}^{1} \subset L^{2}(\Omega) \subset H_{\text {per }}^{-1}$, the semi-scalar product on $H_{\text {per }}^{1}$,

$$
(u, v) \mapsto(\nabla u, \nabla v),
$$

defines a linear operator $\mathcal{A}=-\Delta: D(\mathcal{A}) \rightarrow L^{2}(\Omega)$ with domain $D(\mathcal{A})=H_{\text {per }}^{2}$. We denote $\dot{\mathcal{A}}=-\Delta: D(\dot{\mathcal{A}}) \rightarrow$ $L^{2}(\Omega)$ the restriction of $\mathcal{A}$ to $\dot{L}^{2}(\Omega)$, with domain $D(\dot{\mathcal{A}})=\dot{H}_{\text {per }}^{2}$. We observe that $\dot{\mathcal{A}}$ is a positive self-adjoint operator with compact resolvent so that its powers $\dot{\mathcal{A}}^{s}(s \in \mathbb{R})$ are well-defined and it is possible to prove that $\dot{H}_{p e r}^{m}=D\left(\dot{\mathcal{A}}^{m / 2}\right)(m \in \mathbb{Z})$.

For $m=-1$, we introduce an equivalent and more convenient norm $|\cdot|_{-1}$ on $\dot{H}_{\text {per }}^{-1}$ associated with the inner product

$$
(\dot{u}, \dot{v})_{-1}=\left(\dot{\mathcal{A}}^{-1 / 2} \dot{u}, \dot{\mathcal{A}}^{-1 / 2} \dot{v}\right)
$$

so that for any $\dot{u} \in \dot{H}_{p e r}^{-1}$, we have

$$
|\dot{u}|_{-1}=\left\|\dot{\mathcal{A}}^{-1 / 2} \dot{u}\right\|=\left\|\nabla \dot{\mathcal{A}}^{-1} \dot{u}\right\| .
$$

Similarly, for $m=1$, we will sometimes use the equivalent norm $|\cdot|_{1}$ in $H_{\text {per }}^{1}$ associated with the inner product

$$
(u, v)_{1}=\langle u\rangle\langle v\rangle+(\nabla u, \nabla v) .
$$

Moreover, $\dot{\mathcal{A}}$ defines a continuous bijection from $\dot{H}_{\text {per }}^{m}$ onto $\dot{H}_{\text {per }}^{m-2}$. In particular, for $m=-1$,

$$
(\dot{u}, \dot{v})_{-1}=\left\langle\mathcal{A}^{-1} \dot{u}, \dot{v}\right\rangle_{H_{p e r}^{1}, H_{p e r}^{-1}}=\left\langle\dot{u}, \mathcal{A}^{-1} \dot{v}\right\rangle_{H_{p e r}^{-1}, H_{p e r}^{1}}=\left(\mathcal{A}^{-1 / 2} \dot{u}, \mathcal{A}^{-1 / 2} \dot{v}\right) .
$$

\subsection{Energy solutions}

The nonlinearity $f$ is a polynomial of odd degree whose leading coefficient is positive and which vanishes at 0 ,

$$
f(s)=\sum_{i=1}^{2 p+1} a_{i} s^{i} \quad \forall s \in \mathbb{R} \quad\left(a_{2 p+1}>0\right),
$$

with $p \in \mathbb{N} \backslash\{0\}$ if $d=1$ or $d=2$ and with $p \in\{1,2\}$ if $d=3$. The restriction on $p$ when $d=3$ is due to the use of $H_{\text {per }}^{1}$ conforming finite elements (see $(2.3)$ ). We denote $F$ the antiderivative of $f$ which vanishes at 0 , i.e.,

$$
F(s)=\sum_{i=2}^{2 p+2} \frac{a_{i-1}}{i} s^{i} \quad \forall s \in \mathbb{R} .
$$

We will make use of the Sobolev injection $H_{p e r}^{1} \subset L^{2 p+2}(\Omega)$. In particular, there is a constant $C_{S}=C_{S}(\Omega, p)$ such that

$$
\|u\|_{L^{2 p+2}(\Omega)} \leq C_{S}|u|_{1}, \quad \forall u \in H_{p e r}^{1},
$$

and the map $v \mapsto f(v)$ is Lipschitz continuous on bounded sets of $H_{p e r}^{1}$ with values into $L^{(2 p+2) /(2 p+1)}(\Omega) \subset$ $H_{p e r}^{-1}$. We also have $H_{p e r}^{2} \subset C^{0}(\bar{\Omega})$ with continuous injection. Finally, we note that there exist constants $c_{1} \geq 0$, $c_{2}>0$ and $c_{3} \geq 0$ such that

$$
\begin{gathered}
F(s) \geq 2 s^{2}-c_{1} \quad \forall s \in \mathbb{R}, \\
|f(s)| \leq c_{2} F(s)+c_{3} \quad \forall s \in \mathbb{R} .
\end{gathered}
$$


We point out that the expression (2.2) includes the standard quartic potential (obtained with $p=1$ )

$$
F(s)=\frac{s^{4}}{4}+\frac{(1-\varepsilon)}{2} s^{2} .
$$

In contrast to some authors, we do not assume that $\varepsilon<1$ (we simply have $\varepsilon \in \mathbb{R}$ ).

In [23], a notion of energy solution was introduced. This is based on the following pseudo-energy,

$$
\mathcal{E}(u, v)=E(u)+\frac{\beta}{2}|\dot{v}|_{-1}^{2},
$$

which is well-defined for any $(u, v) \in H_{p e r}^{2} \times H_{p e r}^{-1}$.

Definition 2.1. A pair $\left(u, u_{t}\right)$ is called an energy solution to problem (1.1) and (1.2) if

$$
\begin{gathered}
\left(u, u_{t}\right) \in L^{\infty}\left(\mathbb{R}_{+} ; H_{p e r}^{2} \times H_{p e r}^{-1}\right), \quad u_{t t} \in L^{\infty}\left(\mathbb{R}_{+} ; H_{p e r}^{-4}\right) \\
\mathcal{E}\left(u, u_{t}\right) \in L^{\infty}\left(\mathbb{R}_{+}\right)
\end{gathered}
$$

and the following relations hold

$$
\begin{gathered}
\left\langle\beta u_{t t}+u_{t}\right\rangle=0, \\
\dot{\mathcal{A}}^{-1}\left(\beta u_{t t}+u_{t}\right)+\mathcal{A}^{2} u-2 \mathcal{A} u+f(u)-\langle f(u)\rangle=0, \\
\text { in } \dot{H}_{p e r}^{-2}, \quad \text { a.e. in } \mathbb{R}_{+}, \\
u(0)=u_{0} \text { in } H_{p e r}^{2}, \quad u_{t}(0)=v_{0} \text { in } H_{p e r}^{-1} .
\end{gathered}
$$

Equation (2.10) can be interpreted as a conservation law for the mass. We remark that the initial conditions in (2.12) make sense because the regularity of $\left(u, u_{t}\right)$ in (2.8) ensures the weak continuity $\left(u, u_{t}\right) \in C_{w}\left(\mathbb{R}_{+}, H_{\text {per }}^{2} \times\right.$ $\left.H_{\text {per }}^{-1}\right)$. Here we denote $C_{w}\left(\mathbb{R}_{+}, H_{\text {per }}^{2} \times H_{\text {per }}^{-1}\right)$ as the topological (vector) space of all weakly continuous functions on $\mathbb{R}_{+}$with values in $H_{\text {per }}^{2} \times H_{\text {per }}^{-1}$. With this definition, we have

Theorem $2.2([23])$. For any initial data $\left(u_{0}, v_{0}\right) \in H_{\text {per }}^{2} \times H_{\text {per }}^{-1}$, problem (1.1) and (1.2) has a unique global energy solution $\left(u, u_{t}\right)$. Moreover, any energy solution satisfies the strong time continuity property

$$
u \in C^{2}\left(\mathbb{R}_{+} ; H_{\text {per }}^{-4}\right) \cap C^{1}\left(\mathbb{R}_{+} ; H_{\text {per }}^{-1}\right) \cap C\left(\mathbb{R}_{+} ; H_{\text {per }}^{2}\right),
$$

as well as the following energy identity, for all $t \in \mathbb{R}_{+}$,

$$
\mathcal{E}\left(u(t), u_{t}(t)\right)+\int_{0}^{t}\left|\dot{u}_{t}(s)\right|_{-1}^{2} \mathrm{~d} s=\mathcal{E}\left(u_{0}, v_{0}\right)+\int_{0}^{t}\left\langle v_{0}\right\rangle \mathrm{e}^{-s / \beta}(f(u(s)), 1) \mathrm{d} s
$$

In particular, whenever $\left\langle v_{0}\right\rangle=0$ then the pseudo-energy is nonincreasing.

Remark 2.3. Theorem 2.2 is valid for more general nonlinearities (see Rem. 2.3 and (3.13) in [23]). More precisely, we can take, for instance, a function $f$ satisfying the following assumptions:

$$
\begin{array}{r}
f \in C_{l o c}^{2,1}(\mathbb{R}), f(0)=0, \\
\liminf _{|s| \rightarrow+\infty} f^{\prime}(s)>0, \\
\liminf _{|s| \rightarrow+\infty} \frac{f(s)}{s}=+\infty,
\end{array}
$$

together with a growth condition of polynomial type, namely, there exist positive constants $\gamma_{1}, \gamma_{2}$ such that, for all $s \in \mathbb{R},|f(s)| \leq \gamma_{1} F(s)+\gamma_{2}$. 


\section{THE SPACE SEMI-DISCRETE PROBLEM}

\subsection{The space semi-discrete scheme}

Our space discretization is based on two ideas: first, in view of the time discretization, we write the PDE (1.1) as a first order system; second, in order to use $H_{p e r}^{1}$-conforming finite elements, we split the triLaplacian into three terms, in the spirit of a well-known splitting approach in Cahn-Hilliard type equations (see, e.g., $[10,18,21])$. An advantage of this approach is that the resulting ODE inherits the gradient-like flow structure of the PDE (see (3.5)). On the continuous level, we first obtain the following system, which is (formally) equivalent to (1.1),

$$
\left\{\begin{array}{l}
u_{t}=v \\
\beta v_{t}=-v+\Delta w \\
z=-\Delta u \\
w=-\Delta z+2 \Delta u+f(u) .
\end{array}\right.
$$

Now, let $V_{h}$ denote a finite-dimensional subspace of $H_{p e r}^{1}$ which contains the constants. In applications, $V_{h}$ will be a space of conforming finite elements, typically a $P^{k}$ or $Q^{k}$ finite element space (see Sect. 6). The space $V_{h}$ can also be obtained with a spectral basis.

The space semi-discrete scheme reads: find $u_{h}, v_{h}, z_{h}, w_{h}: \mathbb{R}_{+} \rightarrow V_{h}$ such that

$$
\left\{\begin{array}{l}
\left(\partial_{t} u_{h}, \varphi_{h}\right)=\left(v_{h}, \varphi_{h}\right) \\
\beta\left(\partial_{t} v_{h}, \psi_{h}\right)=-\left(v_{h}, \psi_{h}\right)-\left(\nabla w_{h}, \nabla \psi_{h}\right) \\
\left(z_{h}, \zeta_{h}\right)=\left(\nabla u_{h}, \nabla \zeta_{h}\right) \\
\left(w_{h}, \xi\right)=\left(\nabla z_{h}, \nabla \xi_{h}\right)-2\left(\nabla u_{h}, \nabla \xi_{h}\right)+\left(f\left(u_{h}\right), \xi_{h}\right),
\end{array}\right.
$$

for all $\varphi_{h}, \psi_{h}, \zeta_{h}, \xi_{h}$ in $V_{h}$. This problem is completed with initial conditions

$$
u_{h}(0)=u_{h}^{0}, \quad v_{h}(0)=v_{h}^{0},
$$

where $u_{h}^{0}$ and $v_{h}^{0}$ are given in $V_{h}$.

It will be convenient to work with an appropriate basis of $V_{h}$. For this purpose, let $\left(\mathrm{e}_{h}^{i}\right)_{1 \leq i \leq N_{h}}$ denote an orthonormal basis of $V_{h}$ for the $L^{2}(\Omega)$ scalar product, such that $\mathrm{e}_{h}^{1} \equiv 1$. The integer $N_{h}$ is the dimension of $V_{h}$. To every function $r_{h}=\sum_{i=1}^{N_{h}} r_{i} \mathrm{e}_{h}^{i} \in V_{h}$ corresponds a unique (column) vector $R=\left(r_{1}, \ldots, r_{N_{h}}\right)^{t}$, represented by the corresponding capital letter. We seek

$$
u_{h}(t)=\sum_{i=1}^{N_{h}} u_{i}(t) \mathrm{e}_{h}^{i} \simeq\left(u_{1}(t), \ldots, u_{N_{h}}(t)\right)^{t}=U(t), \quad v_{h} \simeq V, \quad z_{h} \simeq Z, \quad w_{h} \simeq W .
$$

Define $A=\left(A_{i j}\right)_{1 \leq i, j \leq N_{h}}$, where

$$
A_{i j}=\left(\nabla \mathrm{e}_{h}^{i}, \nabla \mathrm{e}_{h}^{j}\right), \quad 1 \leq i, j \leq N_{h},
$$

and let

$$
F_{h}(U)=\left(F\left(u_{h}\right), 1\right), \quad \text { so that } \quad \nabla F_{h}(U)=\left(\left(f\left(u_{h}\right), \mathrm{e}_{h}^{1}\right), \ldots,\left(f\left(u_{h}\right), \mathrm{e}_{h}^{N_{h}}\right)\right)^{t}
$$


By choosing the test functions $\varphi_{h}, \psi_{h}, \zeta_{h}, \xi_{h}$ in (3.1) as the basis functions $\mathrm{e}_{h}^{i}$, we obtain the following equivalent system,

$$
\left\{\begin{array}{l}
U_{t}=V \\
\beta V_{t}=-V-A W \\
Z=A U \\
W=A Z-2 A U+\nabla F_{h}(U) .
\end{array}\right.
$$

Eliminating $V, Z$ and $W$, we see that (3.4) is equivalent to

$$
\beta U_{t t}+U_{t}=-A\left[A^{2} U-2 A U+\nabla F_{h}(U)\right], \quad t \geq 0 .
$$

Since $A$ is a discretization of $-\Delta$, this is a natural space semi-discrete version of (1.1).

Let $U$ denote a solution of (3.5). We notice that the first line and the first column of $A$ are filled with zeros (recall $\mathrm{e}_{h}^{1} \equiv 1$, so that $\left.\nabla \mathrm{e}_{h}^{1} \equiv 0\right)$. Thus, the first component of $U, u_{1}(t)=\left(u_{h}(t), 1\right)$, satisfies

$$
\beta \partial_{t t} u_{1}+\partial_{t} u_{1}=0, \quad t \geq 0
$$

Solving (3.6) with initial conditions $u_{1}(0)=\left(u_{h}^{0}, 1\right)=: u_{1}^{0}$ and $\partial_{t} u_{1}(0)=\left(v_{h}^{0}, 1\right)=: v_{1}^{0}$ yields

$$
\partial_{t} u_{1}(t)=v_{1}^{0} \mathrm{e}^{-t / \beta}=: a_{h}(t) \quad \text { and } \quad u_{1}(t)=\beta v_{1}^{0}+u_{1}^{0}-\beta a_{h}(t) .
$$

For every vector $R=\left(r_{1}, \ldots, r_{N_{h}}\right)^{t} \in \mathbb{R}^{N_{h}}$, we denote $\dot{R}=\left(r_{2}, \ldots, r_{N_{h}}\right)^{t} \in \mathbb{R}^{N_{h}-1}$. Then $\dot{U}$ satisfies

$$
\beta \dot{U}_{t t}+\dot{U}_{t}=-\dot{A}\left[\dot{A}^{2} \dot{U}-2 \dot{A} \dot{U}+\dot{\nabla} F_{h}(U)\right], \quad t \geq 0,
$$

where $\dot{A}$ is the submatrix $\dot{A}=\left(A_{i j}\right)_{2 \leq i, j \leq N_{h}}$, and

$$
\dot{\nabla} F_{h}(U)=\left(\left(f\left(u_{h}\right), \mathrm{e}_{h}^{2}\right), \ldots,\left(f\left(u_{h}\right), \mathrm{e}_{h}^{N_{h}}\right)\right)^{t} .
$$

We can also write $\dot{\nabla} F_{h}(U)=\dot{P}\left(\nabla F_{h}\left(u_{1}(t), \dot{U}\right)\right)$, where $\dot{P}: \mathbb{R}^{N_{h}} \rightarrow \mathbb{R}^{N_{h}-1}$ is the projection on the components $2, \ldots, N_{h}$. This shows that $\dot{\nabla} F_{h}(U)$ is a "non autonomous" function of $\dot{U}$ (recall that $u_{1}(t)$ is determined by (3.7)). For later purpose, we note that by (3.3), $\dot{A}$ is symmetric positive definite: in particular, $\dot{A}$ is invertible.

Conversely, any solution $U$ of (3.7) and (3.8) satisfies (3.4), i.e. that the second equation of (3.4) is satisfied with $V, Z$ and $W$ given by the three other equations of the system (3.4).

\subsection{Discrete energy estimate, existence and uniqueness}

The estimates in this section are essentially the same as those used in the continuous case to establish the existence of energy solutions [23]. However, we derive an energy estimate (3.15) which is new when $v_{1}^{0} \neq 0$.

The standard Euclidean norm in $\mathbb{R}^{N_{h}}$ or $\mathbb{R}^{N_{h}-1}$ will be denoted $|\cdot|$. We also use the following quadratic norm,

$$
|\dot{R}|_{-1}=\left(\dot{R}^{t} \dot{A}^{-1} \dot{R}\right)^{1 / 2}
$$

defined for all $\dot{R} \in \mathbb{R}^{N_{h}-1}$. Notice that $\left|A^{s} U\right|=\left|\dot{A}^{s} \dot{U}\right|\left(s>0, U \in \mathbb{R}^{N_{h}}\right)$. We set

$$
\begin{aligned}
E_{h}(U) & =\frac{1}{2}|A U|^{2}-\left|A^{1 / 2} U\right|^{2}+F_{h}(U), \\
\mathcal{E}_{h}(U, V) & =E_{h}(U)+\frac{\beta}{2}|\dot{V}|_{-1}^{2} .
\end{aligned}
$$


As a shortcut, for a solution $\left(U, U_{t}\right)$ of $(3.5)$, we will write

$$
\mathcal{E}_{h}(t)=\mathcal{E}_{h}\left(U(t), U_{t}(t)\right) .
$$

Notice that by the Cauchy-Schwarz inequality we have

$$
\left|A^{1 / 2} U\right|^{2}=U^{t} A U \leq \frac{1}{4}|A U|^{2}+|U|^{2} .
$$

Then, using also (2.4), we find that

$$
E_{h}(U) \geq \frac{1}{4}|A U|^{2}+|U|^{2}-c_{1}
$$

We first prove the following (compare with (2.13))

Lemma 3.1. Any solution $U \in C^{2}\left([0, T) ; \mathbb{R}^{N_{h}}\right)$ of (3.5) satisfies the energy equality

$$
\frac{\mathrm{d}}{\mathrm{d} t} \mathcal{E}_{h}(t)+\left|\dot{U}_{t}\right|_{-1}^{2}=v_{1}^{0} \mathrm{e}^{-t / \beta}\left(f\left(u_{h}\right), 1\right),
$$

and the energy estimate

$$
\mathcal{E}_{h}(t)+\int_{0}^{t}\left|\dot{U}_{t}(s)\right|_{-1}^{2} \mathrm{~d} s \leq \mathcal{E}_{h}(0) \mathrm{e}^{2 c_{2}\left|v_{1}^{0}\right| \beta}+\left(c_{1} c_{2}+c_{3}\right)\left|v_{1}^{0}\right| \beta \mathrm{e}^{2 c_{2}\left|v_{1}^{0}\right| \beta},
$$

for all $t \in[0, T)$, where $c_{1}, c_{2}$ and $c_{3}$ depend only on $f\left(\right.$ see (2.4) and (2.5)), and where $v_{1}^{0}=\partial_{t} u_{1}(0)$.

Remark 3.2. Whenever $v_{1}^{0}=0$ then the inequality in (3.15) can be replaced by an equality, i.e. for all $t \in \mathbb{R}_{+}$,

$$
\mathcal{E}_{h}(t)+\int_{0}^{t}\left|\dot{U}_{t}(s)\right|_{-1}^{2} \mathrm{~d} s=\mathcal{E}_{h}(0)
$$

(integrate (3.14) between 0 and $t$ ). This is of course a space semi-discrete version of $(2.13)$ when $\left\langle v_{0}\right\rangle=0$.

Proof. Recall that $\partial_{t} u_{1}(t)=v_{1}^{0} \mathrm{e}^{-t / \beta}$ (see (3.7)). On multiplying (3.8) by $\dot{U}_{t}^{t} \dot{A}^{-1}$, and using

$$
\frac{\mathrm{d}}{\mathrm{d} t}\left[F_{h}(U(t))\right]=\left(\nabla F_{h}(U), U_{t}(t)\right)=\sum_{i=1}^{N_{h}} \partial_{t} u_{i}(t)\left(f\left(u_{h}\right), \mathrm{e}_{h}^{i}\right),
$$

we find the energy equality (3.14). Estimate (2.5) yields

$$
\frac{\mathrm{d}}{\mathrm{d} t} \mathcal{E}_{h}(t)+\left|\dot{U}_{t}\right|_{-1}^{2} \leq\left|v_{1}^{0}\right| \mathrm{e}^{-t / \beta}\left(c_{2} F_{h}(U)+c_{3}\right) .
$$

By the Cauchy-Schwarz inequality, we have

$$
2\left|A^{1 / 2} U\right|^{2}=2 U^{t} A U \leq|A U|^{2}+|U|^{2} .
$$

Thus, using (2.4), we get

$$
\begin{aligned}
2 E_{h}(U) & =\left(|A U|^{2}-2\left|A^{1 / 2} U\right|^{2}+|U|^{2}\right)+\left(F_{h}(U)-|U|^{2}\right)+F_{h}(U) \\
& \geq|U|^{2}-c_{1}+F_{h}(U),
\end{aligned}
$$

so that

$$
F_{h}(U) \leq 2 \mathcal{E}_{h}(U, V)+c_{1} .
$$


Therefore we obtain

$$
\frac{\mathrm{d}}{\mathrm{d} t} \mathcal{E}_{h}(t)+\left|\dot{U}_{t}\right|_{-1}^{2} \leq\left|v_{1}^{0}\right| \mathrm{e}^{-t / \beta}\left(2 c_{2} \mathcal{E}_{h}(t)+c_{1} c_{2}+c_{3}\right)
$$

Letting $\eta(t)=\int_{0}^{t} 2 c_{2}\left|v_{1}^{0}\right| \mathrm{e}^{-s / \beta} \mathrm{d} s$ and $c_{3}^{\prime}=c_{1} c_{2}+c_{3}$, Gronwall's lemma yields

$$
\mathcal{E}_{h}(t)+\int_{0}^{t}\left|\dot{U}_{t}(s)\right|_{-1}^{2} \mathrm{e}^{\eta(t)-\eta(s)} \mathrm{d} s \leq \mathcal{E}_{h}(0) \mathrm{e}^{\eta(t)}+\int_{0}^{t} c_{3}^{\prime}\left|v_{1}^{0}\right| \mathrm{e}^{-s / \beta} \mathrm{e}^{\eta(t)-\eta(s)} \mathrm{d} s .
$$

Since $\eta(t)=2 c_{2}\left|v_{1}^{0}\right| \beta\left(1-\mathrm{e}^{-t / \beta}\right) \leq 2 c_{2}\left|v_{1}^{0}\right| \beta$, we deduce the energy estimate (3.15).

Theorem 3.3. For every $U^{0}, V^{0}$ in $\mathbb{R}^{N_{h}}$, there exists a unique solution $U \in C^{2}\left(\mathbb{R}_{+}, \mathbb{R}^{N_{h}}\right)$ of (3.5) such that $U(0)=U^{0}$ and $U_{t}(0)=V^{0}$.

Proof. By the Cauchy-Lipschitz theorem, there exists a unique maximal solution $U \in C^{2}\left(\left[0, T^{+}\right) ; \mathbb{R}^{N_{h}}\right)$ of $(3.5)$ satisfying the given initial conditions. The energy estimate (3.15) shows that $\mathcal{E}_{h}$ is uniformly bounded for $t \geq 0$. By (3.11) and (3.13), $|U|$ and $\left|\dot{U}_{t}\right|_{-1}$ are uniformly bounded for $t \geq 0$. This, together with the estimate (3.7) on the mass, implies that $T^{+}=+\infty$.

\subsection{Discrete operators and $h$-dependent norms}

We assume now that $\left(V_{h}\right)_{h>0}$ is a sequence of subspaces of $H_{p e r}^{1}$ such that:

(H1) for all $h>0, V_{h}$ has finite dimension and contains all the constants;

(H2) for any $\varphi \in H_{p e r}^{1}$, there exists $\varphi_{h} \in V_{h}$ such that $\varphi_{h} \rightarrow \varphi$ (strongly) in $H_{p e r}^{1}$, as $h$ tends to 0 .

As usual in finite element methods, we use the subscript $h$ instead of an integer to denote the sequence $V_{h}(h$ stands for $h_{k}$, where $\left(h_{k}\right)_{k}$ is a sequence of positive real numbers which tends to 0 ).

For the convergence result as $h \rightarrow 0$, it will be useful to have $h$-dependent operators and norms. We denote $\mathcal{A}_{h}: V_{h} \rightarrow V_{h}$ the linear operator such that for any $q_{h} \in V_{h}, \mathcal{A}_{h} q_{h}$ solves

$$
\left(\mathcal{A}_{h} q_{h}, \zeta_{h}\right)=\left(\nabla q_{h}, \nabla \zeta_{h}\right), \quad \forall \zeta_{h} \in V_{h} .
$$

The operator $\mathcal{A}_{h}$ is a discrete Laplacian, $\mathcal{A}_{h} \simeq-\Delta_{h}$. Actually, by using a $L^{2}$-orthonormal basis $\left(\mathrm{e}_{h}^{i}\right)_{1 \leq i \leq N_{h}}$ of $V_{h}$ as in Section 3.1, with $\mathrm{e}_{h}^{1} \equiv 1$, we see that the matrix of $\mathcal{A}_{h}$ is $A(c f .(3.3))$.

Notice that if $q_{h}$ is constant, then $\mathcal{A}_{h} q_{h}=0$ so that $\mathcal{A}_{h}$ is not invertible. In order to define a discrete version of $\dot{\mathcal{A}}^{-1}$, we introduce the subspace

$$
\dot{V}_{h}=\left\{\varphi_{h} \in V_{h}:\left\langle\varphi_{h}\right\rangle=0\right\} .
$$

The bilinear form $(\nabla \cdot, \nabla \cdot)$ is symmetric positive definite on $\dot{V}_{h}$. We can define the operator $\dot{\mathcal{S}}_{h}: \dot{V}_{h} \rightarrow \dot{V}_{h}$ such that for any $\dot{r}_{h} \in \dot{V}_{h}, \dot{\mathcal{S}}_{h} \dot{r}_{h}$ is the unique solution of

$$
\left(\nabla \dot{\mathcal{S}}_{h} \dot{r}_{h}, \nabla \dot{\varphi}_{h}\right)=\left(\dot{r}_{h}, \dot{\varphi}_{h}\right), \forall \dot{\varphi}_{h} \in \dot{V}_{h} .
$$

By choosing $\zeta_{h} \equiv 1$ in (3.17), we see that $\mathcal{A}_{h}\left(V_{h}\right) \subset \dot{V}_{h}$, so that the restriction $\dot{\mathcal{A}}_{h}: \dot{V}_{h} \rightarrow \dot{V}_{h}$ of $\mathcal{A}_{h}$ is well defined. Using (3.17) and (3.18), it is easily seen that $\dot{\mathcal{S}}_{h}=\dot{\mathcal{A}}_{h}^{-1}$.

We also define the $L^{2}$-orthogonal projector $P_{h}: L^{2}(\Omega) \rightarrow V_{h}$, i.e.,

$$
\left(P_{h} q, \varphi_{h}\right)=\left(q, \varphi_{h}\right), \quad \forall q \in L^{2}(\Omega), \forall \varphi_{h} \in V_{h} .
$$

By Pythagoras' theorem and assumption (H2), for any $q \in L^{2}(\Omega)$, we have

$$
\left\|q-P_{h} q\right\|=\inf _{r_{h} \in V_{h}}\left\|q-r_{h}\right\| \rightarrow 0, \text { as } h \rightarrow 0 .
$$


Since $V_{h} \subset H_{\text {per }}^{1}$, the operator $P_{h}$ has a natural extension to $H_{\text {per }}^{-1}$ (also denoted $P_{h}$ ), by setting

$$
P_{h} q \in V_{h}, \quad\left(P_{h} q, \varphi_{h}\right)=\left\langle q, \varphi_{h}\right\rangle_{H_{p e r}^{-1}, H_{p e r}^{1}}, \quad \forall q \in H_{p e r}^{-1}, \forall \varphi_{h} \in V_{h} .
$$

The $H^{1}$-orthogonal projector $\Pi_{h}: H_{p e r}^{1} \rightarrow V_{h}$ is defined as follows: for any $q \in H_{p e r}^{1}, \Pi_{h} q \in V_{h}$ is uniquely defined by

$$
\left\langle\Pi_{h} q\right\rangle=\langle q\rangle \quad \text { and } \quad\left(\nabla \Pi_{h} q, \nabla \varphi_{h}\right)=\left(\nabla q, \nabla \varphi_{h}\right), \quad \forall q \in H_{p e r}^{1}, \forall \varphi_{h} \in V_{h} .
$$

By Pythagoras' theorem and assumption (H2), for any $q \in H_{p e r}^{1}$, we get

$$
\left|q-\Pi_{h} q\right|_{1}=\inf _{r_{h} \in V_{h}}\left|q-r_{h}\right|_{1} \rightarrow 0, \text { as } h \rightarrow 0 .
$$

We point out that the spaces $V_{h}$ and $\dot{V}_{h}$ are invariant by $\Pi_{h}$ and by $P_{h}$.

Since the matrix of $\mathcal{A}_{h}$ is $A$ (in a $L^{2}$-orthonormal basis $\left(\mathrm{e}_{h}^{i}\right)_{1 \leq i \leq N_{h}}$ of $V_{h}$ such that $\mathrm{e}_{h}^{1} \equiv 1$ ), the energy $E_{h}$ from (3.10) can be rewritten

$$
E_{h}\left(u_{h}\right)=\frac{1}{2}\left\|\mathcal{A}_{h} u_{h}\right\|^{2}-\left|\dot{u}_{h}\right|_{1}^{2}+\left(F\left(u_{h}\right), 1\right) ;
$$

the norm $|\cdot|_{-1}$ from (3.9) becomes for any element $\dot{r}_{h} \in \dot{V}_{h}$

$$
\left|\dot{r}_{h}\right|_{-1, h}:=\left(\dot{r}_{h}, \dot{\mathcal{A}}_{h}^{-1} \dot{r}_{h}\right)=\left|\dot{\mathcal{A}}_{h}^{-1} \dot{r}_{h}\right|_{1}
$$

It is a norm associated to the following scalar product on $\dot{V}_{h}$,

$$
\left(\dot{r}_{h}, \dot{q}_{h}\right)_{-1, h}:=\left(\dot{r}_{h}, \dot{\mathcal{A}}_{h}^{-1} \dot{q}_{h}\right)=\left(\dot{\mathcal{A}}_{h}^{-1} \dot{r}_{h}, \dot{\mathcal{A}}_{h}^{-1} \dot{q}_{h}\right)_{1} .
$$

Thus, the discrete pseudo-energy (3.11) reads

$$
\mathcal{E}_{h}\left(u_{h}, v_{h}\right):=E_{h}\left(u_{h}\right)+\frac{\beta}{2}\left|\dot{v}_{h}\right|_{-1, h}^{2}, \quad \forall u_{h}, v_{h} \in V_{h} .
$$

The Cauchy-Schwarz (3.12) inequality gives

$$
\left|\dot{u}_{h}\right|_{1}^{2} \leq \frac{1}{4}\left\|\mathcal{A}_{h} u_{h}\right\|^{2}+\left\|u_{h}\right\|^{2}, \quad \forall u_{h} \in V_{h},
$$

and estimate (3.13) becomes

$$
E_{h}\left(u_{h}\right) \geq \frac{1}{4}\left\|\mathcal{A}_{h} u_{h}\right\|^{2}+\left\|u_{h}\right\|^{2}-c_{1}, \quad \forall u_{h} \in V_{h} .
$$

From (3.22)-(3.24) and $\left\|u_{h}\right\|_{1}^{2}=\left|\dot{u}_{h}\right|_{1}^{2}+\left\|u_{h}\right\|^{2}$, we deduce the useful estimate, valid for all $u_{h}, v_{h} \in V_{h}$,

$$
\mathcal{E}_{h}\left(u_{h}, v_{h}\right) \geq \frac{1}{8}\left\|\mathcal{A}_{h} u_{h}\right\|^{2}+\frac{1}{2}\left\|u_{h}\right\|_{1}^{2}+\frac{\beta}{2}\left|v_{h}\right|_{-1, h}^{2}-c_{1}
$$

\subsection{Convergence as $h \rightarrow 0$}

In order to prove strong convergence as $h \rightarrow 0$, we will need the following two lemmas.

Lemma 3.4. Let $\eta>0$. There exists $C_{\eta}>0$ independent of $h$ such that for all $v_{h} \in V_{h}$,

$$
\left\|v_{h}\right\|_{1} \leq \eta\left\|\mathcal{A}_{h} v_{h}\right\|+C_{\eta}\left\|v_{h}\right\| .
$$


Proof. It is easily seen that for all $v_{h} \in V_{h}$,

$$
\left\|v_{h}\right\|_{1}^{2} \leq \eta^{2}\left\|\mathcal{A}_{h} v_{h}\right\|^{2}+\left(1+\frac{1}{4 \eta^{2}}\right)\left\|v_{h}\right\|^{2}
$$

Indeed, by the Cauchy-Schwarz inequality and Young's inequality,

$$
\left|\dot{v}_{h}\right|_{1}^{2}=\left|\dot{V}^{t} \dot{A} \dot{V}\right| \leq \eta^{2}|\dot{A} \dot{V}|^{2}+\frac{1}{4 \eta^{2}}|\dot{V}|^{2}=\eta^{2}\left\|\mathcal{A}_{h} v_{h}\right\|^{2}+\frac{1}{4 \eta^{2}}\left\|\dot{v}_{h}\right\|^{2} .
$$

Estimate (3.27) follows from $\left\|v_{h}\right\|_{1}^{2}=\left|\dot{v}_{h}\right|^{2}+\left\|v_{h}\right\|^{2}$. We obtain (3.26) by taking the square root of (3.27) and using the inequality $\sqrt{a^{2}+b^{2}} \leq a+b$, valid for all $a, b \geq 0$. The constant $C_{\eta}$ can be chosen as $C_{\eta}=\left(1+1 /\left(4 \eta^{2}\right)\right)^{1 / 2}$.

Lemma 3.5. Let $T>0$ and for every $h>0$, let $u_{h}$ be a function in $C\left([0, T] ; V_{h}\right)$. If $\left(\mathcal{A}_{h} u_{h}\right)_{h>0}$ is bounded in $L^{\infty}\left(0, T ; L^{2}(\Omega)\right)$ and $\left\{u_{h}: h>0\right\}$ is precompact in $C\left([0, T] ; L^{2}(\Omega)\right)$, then $\left\{u_{h}: h>0\right\}$ is precompact in $C\left([0, T] ; H_{p e r}^{1}\right)$.

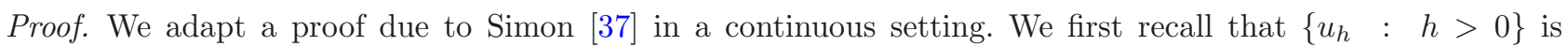
precompact in $C([0, T] ; X)\left(X=L^{2}(\Omega)\right.$ or $X=H_{p e r}^{1}$, with norm $\left.\|\cdot\|_{X}\right)$ if and only if

- for all $\epsilon>0$, there exists a finite subset $\left\{u_{h_{i}}: i=1, \ldots, I\right\}$ such that for all $h>0$, there exists $u_{h_{i}}$ such that $\left\|u_{h}-u_{h_{i}}\right\|_{C([0, T] ; X)} \leq \epsilon$.

Given $\epsilon>0$, there exists a finite subset $\left\{u_{h_{i}}: i=1, \ldots, I\right\}$ such that, for all $h>0$, there exists $u_{h_{i}}$ which fulfills $\left\|u_{h}-u_{h_{i}}\right\|_{C\left([0, T] ; L^{2}(\Omega)\right)} \leq \epsilon$. Then inequality (3.26) implies

$$
\left\|u_{h}-u_{h_{i}}\right\|_{C\left([0, T] ; H_{p e r}^{1}\right)} \leq \eta\left\|\mathcal{A}_{h}\left(u_{h}-u_{h_{i}}\right)\right\|_{C\left([0, T] ; L^{2}(\Omega)\right)}+C_{\eta}\left\|u_{h}-u_{h_{i}}\right\|_{C\left([0, T] ; L^{2}(\Omega)\right)} \leq 2 \eta C+C_{\eta} \epsilon,
$$

where $C$ is a constant independent of $h$ such that $\left\|\mathcal{A}_{h}\right\|_{C\left([0, T] ; L^{2}(\Omega)\right)} \leq C$. Given $\epsilon^{\prime}>0$, for $\eta=\epsilon^{\prime} /(4 C)$ and $\epsilon=\epsilon^{\prime} /\left(2 C_{\eta}\right)$, it yields $\left\|u_{h}-u_{h_{i}}\right\|_{C\left([0, T] ; H_{p e r}^{1}\right)} \leq \epsilon^{\prime}$. This proves the claim.

The convergence result as $h \rightarrow 0$ holds:

Theorem 3.6. Let $\left(u_{0}, v_{0}\right) \in H_{\text {per }}^{2} \times H_{\text {per }}^{-1}$. Assume that $\left(u_{h}^{0}, v_{h}^{0}\right)_{h>0}$ is a family of functions in $V_{h} \times V_{h}$ such that

$$
\begin{gathered}
u_{h}^{0} \rightarrow u_{0} \text { in } H_{p e r}^{1}, \quad \mathcal{A}_{h} u_{h}^{0} \rightarrow \mathcal{A} u_{0} \text { in } L^{2}(\Omega), \\
\left\langle v_{h}^{0}\right\rangle \rightarrow\left\langle v_{0}\right\rangle \text { in } \mathbb{R}, \quad \dot{\mathcal{A}}_{h}^{-1} \dot{v}_{h}^{0} \rightarrow \dot{\mathcal{A}}^{-1} \dot{v}_{0} \text { in } \dot{H}_{p e r}^{1},
\end{gathered}
$$

as $h \rightarrow 0$. Then the solution $\left(u_{h}, \partial_{t} u_{h}\right)$ of the space semi-discrete scheme (3.1) and (3.2) tends to the energy solution $\left(u, u_{t}\right)$ of (1.1) and (1.2) in the following sense,

$$
\begin{aligned}
u_{h} & \rightarrow \text { u weakly } \star \text { in } L^{\infty}\left(\mathbb{R}_{+} ; H_{\text {per }}^{1}\right), \\
u_{h} & \rightarrow \text { ustrongly in } C\left([0, T] ; H_{\text {per }}^{1}\right) \text {, for all } T>0, \\
\mathcal{A}_{h} u_{h} & \rightarrow \mathcal{A} u \text { weakly } \star \text { in } L^{\infty}\left(\mathbb{R}_{+} ; L^{2}(\Omega)\right), \\
\dot{\mathcal{A}}_{h}^{-1} \partial_{t} \dot{u}_{h} & \rightarrow \dot{\mathcal{A}}^{-1} \partial_{t} \dot{u} \text { weakly } \star \text { in } L^{\infty}\left(\mathbb{R}_{+} ; \dot{H}_{\text {per }}^{1}\right) \text { and weakly in } L^{2}\left(\mathbb{R}_{+} ; \dot{H}_{\text {per }}^{1}\right) .
\end{aligned}
$$

Proof. The idea is to use a priori estimates on the mass and on the discrete energy, and to pass to the limit in the equation by a compactness argument. We first consider the conservation law for the mass. By (3.7), we get

$$
a_{h}(t):=\left\langle\partial_{t} u_{h}(t)\right\rangle=\left\langle v_{h}^{0}\right\rangle \mathrm{e}^{-t / \beta}, \quad t \geq 0,
$$


and

$$
\left\langle u_{h}(t)\right\rangle=\beta\left\langle v_{h}^{0}\right\rangle+\left\langle u_{h}^{0}\right\rangle-\beta a_{h}(t), \quad t \geq 0 .
$$

By assumption, $\left\langle u_{h}^{0}\right\rangle \rightarrow\left\langle u_{0}\right\rangle$ and $\left\langle v_{h}^{0}\right\rangle \rightarrow\left\langle v_{0}\right\rangle$ in $\mathbb{R}$, so that $a_{h}$ converges uniformly on $\mathbb{R}_{+}$to the function $a(t):=\left\langle v_{0}\right\rangle \mathrm{e}^{-t / \beta}$, and $\left\langle u_{h}\right\rangle$ converges uniformly on $\mathbb{R}_{+}$to the function $\beta\left\langle v_{0}\right\rangle+\left\langle u_{0}\right\rangle-\beta a(t)$. The estimates below show that $\left(u_{h}\right)_{h>0}$ is bounded in $L^{\infty}\left(\mathbb{R}_{+} ; L^{2}(\Omega)\right)$, so that, up to a subsequence, $u_{h}$ converges weakly $\star$ in $L^{\infty}\left(\mathbb{R}_{+} ; L^{2}(\Omega)\right)$ to some $u$ and so $\left\langle u_{h}\right\rangle \rightarrow\langle u\rangle$ weakly $\star$ in $L^{\infty}\left(\mathbb{R}_{+}\right)$. By uniqueness of the limit, we find

$$
\langle u\rangle=\beta\left\langle v_{0}\right\rangle+\left\langle u_{0}\right\rangle-\beta a(t) .
$$

By differentiating, we recover the conservation law for the mass,

$$
\beta \partial_{t t}\langle u\rangle+\partial_{t}\langle u\rangle=0, \quad t \geq 0 .
$$

We now turn to the energy estimate. As pointed out in Section 3.1, the (unique) solution $\left(u_{h}, \partial_{t} u_{h}\right)$ of $(3.1)$ and (3.2) is in fact a solution $\left(u_{h}, v_{h}, z_{h}, w_{h}\right)$ of (3.1) and (3.2). In particular, $v_{h}=\partial_{t} u_{h}$ and $z_{h}=\mathcal{A}_{h} u_{h}$. We have (recall (3.22))

$$
\mathcal{E}_{h}\left(u_{h}^{0}, v_{h}^{0}\right)=\frac{1}{2}\left\|\mathcal{A}_{h} u_{h}^{0}\right\|^{2}-\left|\dot{u}_{h}^{0}\right|_{1}^{2}+\left(F\left(u_{h}^{0}\right), 1\right)+\frac{\beta}{2}\left|\dot{\mathcal{A}}_{h}^{-1} \dot{v}_{h}^{0}\right|_{1}^{2} .
$$

By using assumptions (3.28) and (3.29) and the Sobolev injection $H_{p e r}^{1} \hookrightarrow L^{2 p+2}(\Omega)$, we see that $\mathcal{E}_{h}\left(u_{h}^{0}, v_{h}^{0}\right)$ is uniformly bounded as $h$ tends to 0 . The energy estimate (3.15) shows that there exists a constant $C$ independent of $h$ such that

$$
\mathcal{E}_{h}\left(u_{h}(t), \partial_{t} u_{h}(t)\right)+\int_{0}^{t}\left|\partial_{t} \dot{u}_{h}(s)\right|_{-1, h}^{2} \mathrm{~d} s \leq C,
$$

for all $t \geq 0$. By (3.25), we obtain that $z_{h}=\mathcal{A}_{h} u_{h}$ and $u_{h}$ are uniformly bounded in $L^{2}(\Omega)$, that $\dot{u}_{h}$ and $\dot{r}_{h}:=\dot{\mathcal{A}}_{h}^{-1} \partial_{t} \dot{u}_{h}$ are uniformly bounded in $\dot{H}_{p e r}^{1}$, and that

$$
\int_{0}^{\infty}\left|\dot{\mathcal{A}}_{h}^{-1} \partial_{t} \dot{u}_{h}(t)\right|_{1}^{2} \mathrm{~d} t \leq C
$$

This implies that $\left\{u_{h}: h>0\right\}$ is precompact in the space $C\left([0, T] ; L^{2}(\Omega)\right)$, for all $T>0$, as a consequence of the Ascoli-Arzelà theorem [37]. Indeed, let $T>0$. The set $\left\{u_{h}: h>0\right\}$ is uniformly bounded on $[0, T]$ with values in $H_{p e r}^{1}$, and $H_{p e r}^{1}$ is compactly embedded into $L^{2}(\Omega)$ by Rellich's theorem. Moreover, for all $0 \leq s \leq t \leq T$, we have

$$
\begin{aligned}
\left\|\dot{u}_{h}(t)-\dot{u}_{h}(s)\right\|^{2} & =2 \int_{s}^{t}\left(\partial_{t} \dot{u}_{h}(\sigma), \dot{u}_{h}(\sigma)-\dot{u}_{h}(s)\right) \mathrm{d} \sigma \\
& =2 \int_{s}^{t}\left(\nabla \dot{r}_{h}(\sigma), \nabla\left[\dot{u}_{h}(\sigma)-\dot{u}_{h}(s)\right]\right) \mathrm{d} \sigma \\
& \leq 4\left\|\dot{r}_{h}\right\|_{L^{\infty}\left(\mathbb{R}_{+} ; H_{\text {per }}^{1}\right)}\left\|\dot{u}_{h}\right\|_{L^{\infty}\left(\mathbb{R}_{+} ; H_{\text {per }}^{1}\right)}|t-s| .
\end{aligned}
$$

Moreover, by (3.31) and the mean value theorem, we find

$$
\left|\left\langle u_{h}(t)\right\rangle-\left\langle u_{h}(s)\right\rangle\right| \leq\left|\left\langle v_{h}^{0}\right\rangle\right||t-s| .
$$

Thus, $\left\{u_{h}: h>0\right\}$ is uniformly equicontinuous on $[0, T]$ with values into $L^{2}(\Omega)$, and therefore precompact in $C\left([0, T] ; L^{2}(\Omega)\right)$, as claimed. Lemma 3.5 and standard compactness arguments [31] show that, up to a 
subsequence, we have the following convergence results, for some $u, z$ and $\dot{r}$,

$$
\begin{aligned}
u_{h} & \rightarrow u \text { weakly } \star \text { in } L^{\infty}\left(\mathbb{R}_{+} ; H_{p e r}^{1}\right), \\
u_{h} & \rightarrow u \text { strongly in } C\left([0, T] ; H_{p e r}^{1}\right), \text { for all } T>0, \\
u_{h} & \rightarrow u \text { a.e. in } \mathbb{R}_{+} \times \Omega, \\
f\left(u_{h}\right) & \rightarrow f(u) \text { weakly in } L^{q}\left(0, T ; L^{q}(\Omega)\right), \text { for all } T>0, \\
z_{h} & \rightarrow z \text { weakly } \star \text { in } L^{\infty}\left(\mathbb{R}_{+} ; L^{2}(\Omega)\right), \\
\dot{r}_{h} & \rightarrow \dot{r} \text { weakly } \star \text { in } L^{\infty}\left(\mathbb{R}_{+} ; \dot{H}_{p e r}^{1}\right), \\
\dot{r}_{h} & \rightarrow \dot{r} \text { weakly in } L^{2}\left(\mathbb{R}_{+} ; \dot{H}_{p e r}^{1}\right),
\end{aligned}
$$

where $q=(2 p+2) /(2 p+1)>1$. It remains to prove that the limit, which has been denoted $u$ for notational convenience, is indeed the energy solution of (1.1) and (1.2).

Let now $\dot{\psi} \in \dot{H}_{\text {per }}^{1}$ and let $\dot{\psi}_{h}=\Pi_{h}(\dot{\psi})$ so that $\dot{\psi}_{h} \rightarrow \dot{\psi}$ strongly in $\dot{H}_{p e r}^{1}$. We have

$$
\left(\partial_{t} \dot{u}_{h}, \dot{\psi}_{h}\right)=\left(\dot{\mathcal{A}}_{h} \dot{r}_{h}, \dot{\psi}_{h}\right)=\left(\nabla \dot{r}_{h}, \nabla \dot{\psi}_{h}\right) \rightarrow(\nabla \dot{r}, \nabla \dot{\psi})
$$

weakly $\star$ in $L^{\infty}\left(\mathbb{R}_{+}\right)$. On the other hand, $\partial_{t}\left(\dot{u}_{h}, \dot{\psi}_{h}\right) \rightarrow \partial_{t}(\dot{u}, \dot{\psi})$ in $\mathcal{D}^{\prime}(0, \infty)$ (i.e. in the sense of distributions), since $\left(\dot{u}_{h}, \dot{\psi}_{h}\right) \rightarrow(\dot{u}, \dot{\psi})$ in $L^{\infty}\left(\mathbb{R}_{+}\right)$weakly $\star$. Thus,

$$
\partial_{t}(\dot{u}, \dot{\psi})=(\nabla \dot{r}, \nabla \dot{\psi})=\langle\dot{v}, \dot{\psi}\rangle_{H_{p e r}^{-1}, H_{p e r}^{1}}
$$

with $\dot{v}=\dot{\mathcal{A}} \dot{r} \in L^{\infty}\left(\mathbb{R}_{+} ; \dot{H}_{p e r}^{-1}\right)$. This shows that $\partial_{t} \dot{u}=\dot{v} \in L^{\infty}\left(\mathbb{R}_{+} ; \dot{H}_{p e r}^{-1}\right)$.

Next, we set $\varphi \in H_{p e r}^{2}$ and we let $\varphi_{h}=\Pi_{h}(\varphi)$, so that $\varphi_{h} \rightarrow \varphi$ strongly in $H_{p e r}^{1}$. Let $\left(\mathrm{e}_{h}^{i}\right)_{1 \leq i \leq N_{h}}$ be an orthonormal basis of $V_{h}$ with $\mathrm{e}_{h}^{1} \equiv 1$. We let $\varphi_{h}=\sum_{i=1}^{N_{h}} \varphi_{i} \mathrm{e}_{h}^{i}$ and $\Phi=\left(\varphi_{1}, \ldots, \varphi_{N_{h}}\right)^{t}$ be the vector associated to $\varphi_{h}$, as in Section 3.1. On multiplying (3.8) by $\dot{\Phi}^{t} \dot{A}^{-1}$ and using $z_{h}=\mathcal{A}_{h} u_{h}$, we find

$$
\beta\left(\partial_{t t} \dot{u}_{h}, \dot{\varphi}_{h}\right)_{-1, h}+\left(\partial_{t} \dot{u}_{h}, \dot{\varphi}_{h}\right)_{-1, h}+\left(\nabla z_{h}, \nabla \varphi_{h}\right)-2\left(\nabla u_{h}, \nabla \varphi_{h}\right)+\left(f\left(u_{h}\right), \dot{\varphi}_{h}\right)=0,
$$

for all $t \geq 0$. We have that

$$
\left(\partial_{t} \dot{u}_{h}, \dot{\varphi}_{h}\right)_{-1, h}=\left(\dot{r}_{h}, \dot{\varphi}_{h}\right) \rightarrow(\dot{r}, \dot{\varphi})=\left(\partial_{t} \dot{u}, \dot{\varphi}\right)_{-1},
$$

by (3.34). The convergence above holds in $L^{\infty}\left(\mathbb{R}_{+}\right)$weak $\star$, so that

$$
\left(\partial_{t t} \dot{u}_{h}, \dot{\varphi}_{h}\right)_{-1, h}=\partial_{t}\left(\partial_{t} \dot{u}_{h}, \dot{\varphi}_{h}\right)_{-1, h} \rightarrow \partial_{t}\left(\partial_{t} \dot{u}, \dot{\varphi}\right)_{-1} \text { in } \mathcal{D}^{\prime}(0, \infty) .
$$

Moreover,

$$
\left(\nabla u_{h}, \nabla \varphi_{h}\right) \rightarrow(\nabla u, \nabla \varphi) \text { in } L^{\infty}\left(\mathbb{R}_{+}\right) \text {weak } \star .
$$

Since $\varphi_{h}=\Pi_{h} \varphi$, we have

$$
\left(\nabla z_{h}, \nabla \varphi_{h}\right)=\left(\nabla z_{h}, \nabla \varphi\right)=\left(z_{h}, \mathcal{A} \varphi\right) \rightarrow(z, \mathcal{A} \varphi),
$$

in $L^{\infty}\left(\mathbb{R}_{+}\right)$weak $\star$. Concerning the last term in (3.35), we have

$$
\left(f\left(u_{h}\right), \dot{\varphi}_{h}\right) \rightarrow(f(u), \dot{\varphi}) \quad \text { weakly in } L^{q}(0, T), \quad \forall T>0 .
$$

Summing up, we have proved that

$$
\beta \partial_{t}\left(\partial_{t} \dot{u}, \dot{\varphi}\right)_{-1}+\left(\partial_{t} \dot{u}, \dot{\varphi}\right)_{-1}+(z, \mathcal{A} \varphi)-2(\nabla u, \nabla \varphi)+(f(u), \varphi)=\langle f(u)\rangle\langle\varphi\rangle .
$$

The equality holds in $\mathcal{D}^{\prime}(0, \infty)$, for all $\varphi \in H_{\text {per }}^{2}$. Moreover, $\partial_{t} \dot{u} \in L^{\infty}\left(\mathbb{R}_{+} ; H_{\text {per }}^{-1}\right), z \in L^{\infty}\left(\mathbb{R}_{+} ; L^{2}(\Omega)\right), u \in$ $L^{\infty}\left(\mathbb{R}_{+} ; H_{\text {per }}^{1}\right)$ and $f(u) \in L^{\infty}\left(\mathbb{R}_{+} ; H_{\text {per }}^{-1}\right)$ so that $\partial_{t t} u$ belongs to $L^{\infty}\left(\mathbb{R}_{+} ; H_{\text {per }}^{-4}\right)$ and

$$
\dot{\mathcal{A}}^{-1}\left(\beta \partial_{t t} \dot{u}+\partial_{t} \dot{u}\right)+\mathcal{A} z-2 \mathcal{A} u+f(u)-\langle f(u)\rangle=0
$$


in $\dot{H}_{\text {per }}^{-2}$, a.e. in $\mathbb{R}_{+}$. Now, recall that $z_{h}=\mathcal{A}_{h} u_{h}$. Let $\zeta \in H_{\text {per }}^{1}$ and $\zeta_{h}=\Pi_{h} \zeta$, so that $\zeta_{h} \rightarrow \zeta$ strongly in $H_{\text {per }}^{1}$. We have

$$
\left(z_{h}, \zeta_{h}\right)=\left(\mathcal{A}_{h} u_{h}, \zeta_{h}\right)=\left(\nabla u_{h}, \nabla \zeta_{h}\right) \rightarrow(\nabla u, \nabla \zeta)
$$

on the one hand, and $\left(z_{h}, \zeta_{h}\right) \rightarrow(z, \zeta)$, on the other hand. Thus, we deduce

$$
(z, \zeta)=(\nabla u, \nabla \zeta)
$$

in $L^{\infty}\left(\mathbb{R}_{+}\right)$. The equality holds for every $\zeta \in H_{\text {per }}^{1}$, so $z=\mathcal{A} u, u \in L^{\infty}\left(\mathbb{R}_{+} ; H_{\text {per }}^{2}\right)$ and $\left(u, u_{t}\right)$ is an energy solution of (1.1). Finally, we check that $\left(u, u_{t}\right)$ satisfies the correct initial conditions. By (3.33) and (3.28), $u(0)=u_{0}$ in $L^{2}(\Omega)$. Let $T>0$ and choose $g \in C^{1}([0, T])$ such that $g(0)=1$ and $g(T)=0$. On multiplying (3.35) by $g(t)$, integrating between 0 and $T$, and using an integration by parts for the first term, we find

$$
\begin{aligned}
\beta\left(\partial_{t} \dot{u}_{h}(0), \dot{\varphi}_{h}(0)\right)_{-1, h}= & -\int_{0}^{T} \beta\left(\partial_{t} \dot{u}_{h}, \dot{\varphi}_{h}\right)_{-1, h} g^{\prime}(t) \mathrm{d} t \\
& +\int_{0}^{T}\left(\left(\partial_{t} \dot{u}_{h}, \dot{\varphi}_{h}\right)_{-1, h}+\left(\nabla z_{h}, \nabla \varphi_{h}\right)-2\left(\nabla u_{h}, \nabla \varphi_{h}\right)+\left(f\left(u_{h}\right), \dot{\varphi}_{h}\right)\right) g(t) \mathrm{d} t .
\end{aligned}
$$

Letting $h \rightarrow 0$, using (3.29) and (3.36), we obtain

$$
\beta\left(\dot{v}_{0}, \dot{\varphi}(0)\right)_{-1}=-\int_{0}^{T} \beta\left(\partial_{t} \dot{u}, \dot{\varphi}\right)_{-1} g^{\prime}(t) \mathrm{d} t-\int_{0}^{T} \beta \partial_{t}\left(\partial_{t} \dot{u}, \dot{\varphi}\right)_{-1} g(t) \mathrm{d} t .
$$

Performing an integration by parts in the last integral yields

$$
\beta\left(\dot{v}_{0}, \dot{\varphi}(0)\right)_{-1}=\beta\left(\partial_{t} \dot{u}(0), \dot{\varphi}(0)\right)_{-1} .
$$

The test function $\varphi$ is arbitrary in $H_{p e r}^{2}$, so $\partial_{t} \dot{u}(0)=\dot{v}_{0}$ in $H_{\text {per }}^{-1}$. We have seen (recall (3.30)) that $\left\langle\partial_{t} u_{h}(t)\right\rangle$ converges uniformly on $\mathbb{R}_{+}$to the function $t \mapsto\left\langle v_{0}\right\rangle \mathrm{e}^{-t / \beta}$, and in $\mathcal{D}^{\prime}(0, \infty)$ to $\partial_{t}\langle u\rangle$, so that $\left\langle\partial_{t} u(0)\right\rangle=\left\langle v_{0}\right\rangle$. This shows that $\left(u, u_{t}\right)$ is the energy solution of (1.1) and (1.2). By uniqueness of the limit $\left(u, u_{t}\right)$, the whole family $\left(u_{h}, \partial_{t} u_{h}\right)$ converges to $\left(u, u_{t}\right)$.

Remark 3.7. Let $\left(u_{0}, v_{0}\right) \in H_{\text {per }}^{2} \times H_{\text {per }}^{-1}$. If $u_{h}^{0}=\Pi_{h}\left(u_{0}\right)$ and $v_{h}^{0}=P_{h}\left(v_{0}\right)$, then assumptions (3.28) and (3.29) are satisfied. Indeed, for all $\varphi_{h} \in V_{h}$,

$$
\left(\mathcal{A} u_{0}, \varphi_{h}\right)=\left(\nabla u_{0}, \nabla \varphi_{h}\right)=\left(\nabla \Pi_{h} u_{0}, \nabla \varphi_{h}\right)=\left(\mathcal{A}_{h}\left(\Pi_{h} u_{0}\right), \varphi_{h}\right) .
$$

Then $\mathcal{A}_{h}\left(\Pi_{h} u_{0}\right)=P_{h}\left(\mathcal{A} u_{0}\right)$ and by (3.19) we obtain

$$
\mathcal{A}_{h}\left(\Pi_{h} u_{0}\right) \rightarrow \mathcal{A} u_{0} \text { in } L^{2}(\Omega), \text { as } h \rightarrow 0 .
$$

By definition, observe that

$$
\left\langle P_{h}\left(v_{0}\right)\right\rangle=\left(P_{h}\left(v_{0}\right), 1\right)=\left\langle v_{0}, 1\right\rangle_{H_{p e r}^{-1}, H_{p e r}^{1}}=\left\langle v_{0}\right\rangle .
$$

Moreover, for all $\varphi_{h} \in V_{h}$, we have

$$
\begin{aligned}
\left(P_{h}\left(\dot{v}_{0}\right), \dot{\varphi}_{h}\right) & =\left\langle\dot{v}_{0}, \dot{\varphi}_{h}\right\rangle_{H_{p e r}^{-1}, H_{p e r}^{1}}=\left(\nabla \dot{\mathcal{A}}^{-1} \dot{v}_{0}, \nabla \dot{\varphi}_{h}\right) \\
& =\left(\nabla \Pi_{h}\left(\dot{\mathcal{A}}^{-1} \dot{v}_{0}\right), \nabla \dot{\varphi}_{h}\right)=\left(\dot{\mathcal{A}}_{h}\left(\Pi_{h}\left(\dot{\mathcal{A}}^{-1} \dot{v}_{0}\right)\right), \dot{\varphi}_{h}\right),
\end{aligned}
$$

so that

$$
P_{h}\left(\dot{v}_{0}\right)=\dot{\mathcal{A}}_{h}\left(\Pi_{h}\left(\dot{\mathcal{A}}^{-1} \dot{v}_{0}\right)\right)
$$

Thus, thanks to (3.21), we deduce

$$
\dot{\mathcal{A}}_{h}^{-1}\left(P_{h}\left(\dot{v}_{0}\right)\right)=\Pi_{h}\left(\dot{\mathcal{A}}^{-1} \dot{v}_{0}\right) \rightarrow \dot{\mathcal{A}}^{-1} \dot{v}_{0} \text { in } H_{p e r}^{1}
$$


Remark 3.8. Theorem 3.6 holds for more general linearities. For instance, we can take $f$ satisfying assumptions (2.14) and (2.15) together with the following growth conditions: there exist a real number $p \in[1, \infty)$ (with $p \leq 2$ when $d=3$ ) and positive constants $\alpha_{1}, \alpha_{2}, \ldots, \alpha_{10}$ such that, for all $s \in \mathbb{R}$,

$$
\begin{array}{r}
\alpha_{1}|s|^{2 p+2}-\alpha_{2} \leq s f(s) \leq \alpha_{3}|s|^{2 p+2}+\alpha_{4}, \\
\alpha_{5}|s|^{2 p+2}-\alpha_{6} \leq F(s) \leq \alpha_{7}|s|^{2 p+2}+\alpha_{8}, \\
\left|f^{\prime}(s)\right| \leq \alpha_{9}|s|^{2 p}+\alpha_{10} .
\end{array}
$$

\section{THE FUlly DisCRETE PROBLEM}

\subsection{The fully discrete scheme}

For the time discretization, we use the scheme of Gomez and Hughes [19], which can be seen as a CrankNicolson scheme with a second-order stabilization term. The stabilization term is based on the following decomposition:

(H3) $F=F_{+}+F_{-}$, where $F_{+}$and $F_{-}$are polynomials such that $F_{+}^{(4)} \geq 0, F_{-}^{(4)} \leq 0$, and $\operatorname{deg}\left(F_{-}\right)<\operatorname{deg}(F)$ (here, deg denotes the degree of the polynomial and $F_{ \pm}^{(4)}$ denotes the fourth derivative of $F_{ \pm}$).

As a consequence, $\operatorname{deg}\left(F_{+}\right)=\operatorname{deg}(F)$ and $F_{+}, F$ have the same leading coefficient. We denote $f=F^{\prime}=f_{+}+f_{-}$, where $f_{+}=F_{+}^{\prime}$ and $f_{-}=F_{-}^{\prime}$. For the energy estimate, we will use the fact that there exist two constants $c_{5}>0$ and $c_{6} \geq 0$ which depend only on $f$ and on the decomposition $f=f_{+}+f_{-}$such that

$$
\frac{1}{2}(|f(r)|+|f(s)|)+\frac{1}{12}(s-r)^{2}\left(\left|f_{+}^{\prime \prime}(r)\right|+\left|f_{-}^{\prime \prime}(s)\right|\right) \leq c_{5}(F(r)+F(s))+c_{6},
$$

for all $r, s \in \mathbb{R}$. This estimate follows from the fact that $F$ is a polynomial of even degree higher than $f$.

Remark 4.1. A decomposition (H3) is always possible for a polynomial potential such as (2.2). Indeed, for a quartic polynomial (for instance (2.6)), we can always choose $F_{+}=F$ and $F_{-}=0$. For a polynomial with higher degree, we notice that $F^{(4)}$, being a polynomial of even degree with strictly positive leading coefficient, is bounded from below, i.e.

$$
F^{(4)}(s) \geq-c_{4} \quad \forall s \in \mathbb{R}
$$

for some constant $c_{4} \geq 0$. A possible (but not unique!) choice is then $F_{+}^{(4)}=F^{(4)}+c_{4}$ and $F_{-}^{(4)}=-c_{4}$, i.e. $F_{+}(s)=F(s)+c_{4} s^{4} / 24$ and $F_{-}(s)=-c_{4} s^{4} / 24$.

We use the same notation as in Section 3. In particular, $V_{h}$ is a family of finite-dimensional subspaces of $H_{p e r}^{1}$ which satisfies assumptions (H1) and (H2).

Let $\tau>0$ denote the time step, and $\left(u_{h}^{0}, v_{h}^{0}\right)$ in $V_{h} \times V_{h}$ the initial datum. The fully discrete scheme reads: for $n \geq 0$, find $\left(u^{n+1}, v^{n+1}, z^{n+1}, w^{n+1}\right) \in\left(V_{h}\right)^{4}$ such that

$$
\left\{\begin{array}{l}
\left(\left(u_{h}^{n+1}-u_{h}^{n}\right) / \tau, \varphi_{h}\right)=\left(v_{h}^{n+1 / 2}, \varphi_{h}\right) \\
\beta\left(\left(v_{h}^{n+1}-v_{h}^{n}\right) / \tau, \psi_{h}\right)=-\left(v_{h}^{n+1 / 2}, \psi_{h}\right)-\left(\nabla w_{h}^{n+1}, \nabla \psi_{h}\right) \\
\left(z_{h}^{n+1}, \zeta_{h}\right)=\left(\nabla u_{h}^{n+1 / 2}, \nabla \zeta_{h}\right) \\
\left(w_{h}^{n+1}, \xi_{h}\right)=\left(\nabla z_{h}^{n+1}, \nabla \xi_{h}\right)-2\left(\nabla u_{h}^{n+1 / 2}, \nabla \xi_{h}\right)+\left(\left(f\left(u_{h}^{n}\right)+f\left(u_{h}^{n+1}\right)\right) / 2, \xi_{h}\right) \\
\quad-\frac{1}{12}\left(\left(u_{h}^{n+1}-u_{h}^{n}\right)^{2}\left(f_{+}^{\prime \prime}\left(u_{h}^{n}\right)+f_{-}^{\prime \prime}\left(u_{h}^{n+1}\right)\right), \xi_{h}\right),
\end{array}\right.
$$

for all $\varphi_{h}, \psi_{h}, \zeta_{h}, \xi_{h}$ in $V_{h}$. Here, we have denoted

$$
u_{h}^{n+1 / 2}=\left(u_{h}^{n+1}+u_{h}^{n}\right) / 2 \quad \text { and } \quad v_{h}^{n+1 / 2}=\left(v_{h}^{n+1}+v_{h}^{n}\right) / 2 .
$$


Notice that $z_{h}^{0}$ and $w_{h}^{0}$ do not need to be defined. In fact, $z_{h}^{n+1}$ (resp. $w_{h}^{n+1}$ ) is a second-order (in time) approximation of $z_{h}\left(t_{n+1 / 2}\right)$ (resp. $w_{h}\left(t_{n+1 / 2}\right)$ ), where $t_{n+1 / 2}=(n+1 / 2) \tau$. Actually $z_{h}^{n+1 / 2}$ and $w_{h}^{n+1 / 2}$ would have been a more appropriate notation, but are not used for notational convenience.

Let $\left(e_{h}^{i}\right)_{1 \leq i \leq N_{h}}$ be an $L^{2}$-orthonormal basis of $V_{h}$, with $e_{h}^{1} \equiv 1$, so that we have the identification $V_{h} \ni u_{h} \simeq$ $U \in \mathbb{R}^{N_{h}}$. In $\mathbb{R}^{N_{h}}$, the scheme reads: let $U^{0}, V^{0}$ in $\mathbb{R}^{N_{h}}$ and for $n \geq 0$ find $\left(U^{n+1}, V^{n+1}, Z^{n+1}, W^{n+1}\right) \in\left(\mathbb{R}^{N_{h}}\right)^{4}$ which solves

$$
\left\{\begin{array}{l}
\left(U^{n+1}-U^{n}\right) / \tau=V^{n+1 / 2} \\
\beta\left(V^{n+1}-V^{n}\right) / \tau=-V^{n+1 / 2}-A W^{n+1} \\
Z^{n+1}=A U^{n+1 / 2} \\
W^{n+1}=A Z^{n+1}-2 A U^{n+1 / 2}+\left(\nabla F_{h}\left(U^{n}\right)+\nabla F_{h}\left(U^{n+1}\right)\right) / 2-G\left(U^{n}, U^{n+1}\right),
\end{array}\right.
$$

where

$$
G\left(U^{n}, U^{n+1}\right)=\frac{1}{12}\left(\left(\left(u_{h}^{n+1}-u_{h}^{n}\right)^{2}\left(f_{+}^{\prime \prime}\left(u_{h}^{n}\right)+f_{-}^{\prime \prime}\left(u_{h}^{n+1}\right)\right), \mathrm{e}_{h}^{i}\right)\right)_{1 \leq i \leq N_{h}} .
$$

On eliminating $Z^{n+1}$ and $W^{n+1}$, the scheme becomes

$$
\left\{\begin{array}{l}
\left(U^{n+1}-U^{n}\right) / \tau-V^{n+1 / 2}=0 \\
\beta\left(V^{n+1}-V^{n}\right) / \tau+V^{n+1 / 2}+A\left(A^{2} U^{n+1 / 2}-2 A U^{n+1 / 2}\right) \\
\quad+A\left(\left(\nabla F_{h}\left(U^{n}\right)+\nabla F_{h}\left(U^{n+1}\right)\right) / 2-G\left(U^{n}, U^{n+1}\right)\right)=0 .
\end{array}\right.
$$

In Section 6.1, a numerical example indicates that our fully discrete scheme (4.2) has a second order convergence error in time (and also in space if $V_{h}$ is the space of $P^{1}$ finite elements). By arguing as in Gomez and Hughes [19], we check here the following.

Proposition 4.2. The scheme has a second order consistency error in time, i.e. any solution of the space semi-discrete problem (3.4) satisfies the fully discrete scheme (4.5) with order $O\left(\tau^{2}\right)$.

Proof. Let $(U, V)$ be a solution of (3.4) on a finite time interval $[0, T]$. Since $f$ is a polynomial, $F_{h}$ is a polynomial (of $N_{h}$ variables) and by a bootstrap argument, we know that $(U, V) \in C^{\infty}\left([0, T] ; \mathbb{R}^{N_{h}} \times \mathbb{R}^{N_{h}}\right)$. In the time discrete scheme (4.5), we replace $U^{n}, U^{n+1}, V^{n}, V^{n+1}$ by $U\left(t_{n}\right), U\left(t_{n+1}\right), V\left(t_{n}\right), V\left(t_{n+1}\right)$ respectively. Now we notice that the midpoint scheme is the same as (4.5) without the term $G\left(U^{n}, U^{n+1}\right)$. Standard calculations show that the midpoint scheme has a local truncation error (or consistency error) in $O\left(\tau^{2}\right)$. It is therefore sufficient to show that $\left|G\left(U\left(t_{n}\right), U\left(t_{n+1}\right)\right)\right|_{\infty}=O\left(\tau^{2}\right)$, where $|\cdot|_{\infty}$ denotes the maximum norm in $\mathbb{R}^{N_{h}}$. This follows from definition (4.4). Indeed, since $\operatorname{deg}\left(f_{-}^{\prime \prime}\right)<\operatorname{deg}\left(f_{+}^{\prime \prime}\right) \leq 2 p-1$ (recall assumption (H3)), by the triangle inequality and Hölder's inequality we have

$$
\begin{array}{r}
\left\|f_{+}^{\prime \prime}\left(u_{h}\left(t_{n}\right)\right)\right\|_{L^{(2 p+2) /(2 p-1)}(\Omega)}+\left\|f_{-}^{\prime \prime}\left(u_{h}\left(t_{n+1}\right)\right)\right\|_{L^{(2 p+2) /(2 p-1)}(\Omega)} \\
\leq C\left(\left\|u_{h}\left(t_{n}\right)\right\|_{L^{2 p+2}(\Omega)}^{2 p-1}+\left\|u_{h}\left(t_{n+1}\right)\right\|_{L^{2 p+2}(\Omega)}^{2 p-1}+1\right) \leq C_{h},
\end{array}
$$

where $C_{h}$ depends on the maximum value of $t \mapsto\left\|u_{h}(t)\right\|_{1}$ on $[0, T]$ (recall (2.3)). Thus, by Hölder's inequality,

$$
\left|G\left(U\left(t_{n}\right), U\left(t_{n+1}\right)\right)\right|_{\infty} \leq \frac{C_{h}}{12}\left\|u_{h}\left(t_{n+1}\right)-u_{h}\left(t_{n}\right)\right\|_{L^{2 p+2}(\Omega)}^{2} \max _{1 \leq i \leq N_{h}}\left\|e_{h}^{i}\right\|_{L^{2 p+2}(\Omega)} .
$$

Using (2.3) again and the mean inequality, we obtain $\left|G\left(U\left(t_{n}\right), U\left(t_{n+1}\right)\right)\right|_{\infty}=O\left(\tau^{2}\right)$, as claimed. Notice that the constant in the consistency error depends on $h$, on $T$, and on maximum norms of derivatives of $U, V$ up to order 3 . 


\subsection{Existence, discrete energy estimate and uniqueness}

Let us prove the following.

Theorem 4.3 (Existence for any $\tau$ ). For any $\left(u_{h}^{0}, v_{h}^{0}\right) \in V_{h} \times V_{h}$, there exists at least one sequence $\left(u_{h}^{n}, v_{h}^{n}, z_{h}^{n}, w_{h}^{n}\right)_{n \geq 1}$ in $\left(V_{h}\right)^{4}$ which complies with $(4.2)$.

Proof. We work with the $\mathbb{R}^{N_{h}}$ version (4.5). We will show that this problem is variational, and that we can find $U^{n+1}$ by a minimization procedure. Let $\left(U^{n}, V^{n}\right)$ be fixed in $\mathbb{R}^{N_{h}}$. Consider the polynomial of two variables

$$
g(r, s)=\frac{1}{12}(s-r)^{2}\left(f_{+}^{\prime \prime}(r)+f_{-}^{\prime \prime}(s)\right) \quad(r, s \in \mathbb{R}) .
$$

By assumption (H3), we have $\operatorname{deg}\left(f_{-}\right)<\operatorname{deg}(f)$ and $\operatorname{deg}\left(f_{+}\right)=\operatorname{deg}(f)$ so $\operatorname{deg}\left(f_{-}^{\prime \prime}\right)<\operatorname{deg}(f)-2$, and $\operatorname{deg}\left(f_{+}^{\prime \prime}\right)=$ $\operatorname{deg}(f)-2$. Thus, $g$ is a polynomial of total degree less than or equal to $2 p+1$, and its partial degree with respect to the variable $s$ is strictly less than $2 p+1$. We can write

$$
g(r, s)=\sum_{k, l} b_{k, l} r^{k} s^{l}
$$

for coefficients $b_{k, l} \in \mathbb{R}$, where $0 \leq k \leq 2 p+1,0 \leq l<2 p+1, k+l \leq 2 p+1$ and either $k \leq 2$ or $l \leq 2$. Let us set now

$$
h(r, s)=\sum_{k, l} b_{k, l} r^{k} \frac{s^{l+1}}{l+1},
$$

so that $\partial_{s} h(r, s)=g(r, s)$. We define $H_{h}^{n}(U)=\left(h\left(u_{h}^{n}, u_{h}\right), 1\right)$ with $u_{h} \simeq U$, so that

$$
\nabla H_{h}^{n}(U)=\left(g\left(u_{h}^{n}, u_{h}\right), e_{i}\right)_{1 \leq i \leq N_{h}}=G\left(U^{n}, U\right) .
$$

By (4.7) and Hölder's inequality, we get

$$
\left|H_{h}^{n}(U)\right| \leq C_{n}\left(\left\|u_{h}\right\|_{L^{2 p+2}(\Omega)}^{2 p+1}+1\right) \quad \forall U \in \mathbb{R}^{N_{h}},
$$

where the constant $C_{n}$ depends on $\left\|u_{h}^{n}\right\|_{L^{2 p+2}(\Omega)}$. Now, by eliminating $V^{n+1}$, we find that (4.5) is equivalent to

$$
\begin{aligned}
\frac{\beta}{\tau}\left(\frac{2\left(U^{n+1}-U^{n}\right)}{\tau}-\right. & \left.V^{n}\right)+\frac{U^{n+1}-U^{n}}{\tau} \\
& +A\left[A^{2} \frac{\left(U^{n+1}+U^{n}\right)}{2}-2 A \frac{\left(U^{n+1}+U^{n}\right)}{2}+\frac{\nabla F_{h}\left(U^{n}\right)+\nabla F_{h}\left(U^{n+1}\right)}{2}-\nabla H_{h}^{n}\left(U^{n+1}\right)\right]=0 .
\end{aligned}
$$

Writing $U=\left(u_{1}, \dot{U}\right)$, we see that this is equivalent to

$$
\begin{aligned}
& \frac{\beta}{\tau}\left(\frac{2\left(u_{1}^{n+1}-u_{1}^{n}\right)}{\tau}-v_{1}^{n}\right)+\frac{u_{1}^{n+1}-u_{1}^{n}}{\tau}=0, \\
& \frac{\beta \dot{A}^{-1}}{\tau}\left(\frac{2\left(\dot{U}^{n+1}-\dot{U}^{n}\right)}{\tau}-\dot{V}^{n}\right)+\dot{A}^{-1} \frac{\left(\dot{U}^{n+1}-\dot{U}^{n}\right)}{\tau}+\dot{A}^{2} \frac{\left(\dot{U}^{n+1}+\dot{U}^{n}\right)}{2} \\
& -2 \dot{A} \frac{\left(\dot{U}^{n+1}+\dot{U}^{n}\right)}{2}+\frac{\dot{\nabla} F_{h}\left(U^{n}\right)+\dot{\nabla} F_{h}\left(U^{n+1}\right)}{2}-\dot{\nabla} H_{h}^{n}\left(U^{n+1}\right)=0 .
\end{aligned}
$$


The first equation determines $u_{1}^{n+1}$ uniquely. The second equation can be solved by letting $\dot{U}^{n+1}$ be a minimizer on $\mathbb{R}^{N_{h}-1}$ of the function

$$
\begin{aligned}
\mathcal{G}: \dot{U} \mapsto & \frac{\beta}{\tau^{2}}\left|\dot{U}-\dot{U}^{n}\right|_{-1}^{2}-\frac{\beta}{\tau}\left(\dot{V}^{n}\right)^{t} \dot{A}^{-1} \dot{U}+\frac{1}{2 \tau}\left|\dot{U}-\dot{U}^{n}\right|_{-1}^{2}+\frac{1}{4}\left|\dot{A}\left(\dot{U}+\dot{U}^{n}\right)\right|^{2} \\
& -\frac{1}{2}\left|\dot{A}^{1 / 2}\left(\dot{U}+\dot{U}^{n}\right)\right|^{2}+\frac{\left(\dot{\nabla} F_{h}\left(U^{n}\right)\right)^{t}}{2} \dot{U}+\frac{\tilde{F}_{h}^{n}(\dot{U})}{2}-\tilde{H}_{h}^{n}(\dot{U}),
\end{aligned}
$$

where

$$
\tilde{F}_{h}^{n}(\dot{U})=F_{h}\left(u_{1}^{n+1}, \dot{U}\right), \quad \tilde{H}_{h}^{n}(\dot{U})=H_{h}^{n}\left(u_{1}^{n+1}, \dot{U}\right)
$$

By (2.2), we deduce

$$
\tilde{F}_{h}^{n}(\dot{U}) \geq \frac{a_{2 p+1}}{2 p+2}\left\|u_{h}\right\|_{L^{2 p+2}(\Omega)}^{2 p+2}-C_{p}^{\prime}\left(\left\|u_{h}\right\|_{L^{2 p+2}(\Omega)}^{2 p+1}+1\right), \quad \forall \dot{U} \in \mathbb{R}^{N_{h}-1},
$$

where the constant $C_{p}^{\prime}$ depends only on the coefficients of $F$. Thus, by (4.8), we find

$$
\mathcal{G}(\dot{U}) \geq \frac{a_{2 p+1}}{2(2 p+2)}\left\|u_{h}\right\|_{L^{2 p+2}(\Omega)}^{2 p+2}-\left(C_{n}+\frac{C_{p}^{\prime}}{2}\right)\left\|u_{h}\right\|_{L^{2 p+2}(\Omega)}^{2 p+1}-c\left\|\dot{u}_{h}\right\|^{2}-c^{\prime}\left\|\dot{u}_{h}\right\|-c^{\prime \prime},
$$

where $c, c^{\prime}, c^{\prime \prime} \geq 0$ depend on $h, F$ and $u_{h}^{n}$. For the quadratic term, we used that all norms are equivalent in $\mathbb{R}^{N_{h}-1}$. Using this argument again, we see that $\mathcal{G}(\dot{U})$ tends to $+\infty$ as $|\dot{U}|$ tends to $+\infty$. By a standard compactness argument, the continuous function $\mathcal{G}$ has a minimizer in $\mathbb{R}^{N_{h}-1}$. The proof is complete.

The behavior of $\left(u_{1}^{n}, v_{1}^{n}\right)$ is straightforward, thanks to a discrete conservation law for the mass. Indeed, choosing $\psi_{h}=1$ in the second equation of (4.2), we find

$$
v_{1}^{n+1}=q v_{1}^{n}, \quad \text { with } \quad q=q(\beta, \tau)=\frac{2 \beta-\tau}{2 \beta+\tau} .
$$

Thus, we obtain

$$
v_{1}^{n}=q^{n} v_{1}^{0} .
$$

We also have $v_{1}^{n+1 / 2}=q v_{1}^{n-1 / 2}$, so that $v_{1}^{n+1 / 2}=q^{n} v_{1}^{1 / 2}$. Notice that $|q|<1$, since $\beta>0$ and $\tau>0$, so that $v_{1}^{n} \rightarrow 0$. If $\tau>2 \beta$, then $q<0$.

On choosing $\varphi_{h}=1$ in the first equation of (4.2), we find

$$
u_{1}^{n+1}=u_{1}^{n}+\tau v_{1}^{n+1 / 2} .
$$

By induction, we deduce

$$
u_{1}^{n}=u_{1}^{0}+\tau\left(\sum_{k=0}^{n-1} q^{k}\right) v_{1}^{1 / 2}=u_{1}^{0}+\tau \frac{1-q^{n}}{1-q} v_{1}^{1 / 2} .
$$

For the energy estimate, we will need a technical lemma, adapted from [19]:

Lemma 4.4. Let $g \in C^{3}([0,1] ; \mathbb{R})$. Then the following identities hold

$$
\begin{aligned}
& \int_{0}^{1} g(s) \mathrm{d} s=\frac{1}{2}(g(0)+g(1))-\frac{1}{12} g^{\prime \prime}(0)-\frac{1}{2} \int_{0}^{1} k_{2}^{+}(\sigma) g^{(3)}(\sigma) \mathrm{d} \sigma, \\
& \int_{0}^{1} g(s) \mathrm{d} s=\frac{1}{2}(g(0)+g(1))-\frac{1}{12} g^{\prime \prime}(1)+\frac{1}{2} \int_{0}^{1} k_{2}^{-}(\sigma) g^{(3)}(\sigma) \mathrm{d} \sigma,
\end{aligned}
$$

where $k_{2}^{+}(\sigma)=(1-\sigma)^{2}(2 \sigma+1) / 6, k_{2}^{-}(\sigma)=\sigma^{2}(3-2 \sigma) / 6$ and $g^{(3)}$ denotes the third derivative of $g$. In particular, $k_{2}^{+}(\sigma) \geq 0$ and $k_{2}^{-}(\sigma) \geq 0$ for all $\sigma \in[0,1]$. 
Proof. We prove (4.15) (the proof of (4.14) is similar). For a function $\varphi \in C^{2}([0,1])$, let

$$
\operatorname{Err}(\varphi)=\int_{0}^{1} \varphi(s) \mathrm{d} s-\left(\frac{1}{2}(\varphi(0)+\varphi(1))-\frac{1}{12} \varphi^{\prime \prime}(1)\right)
$$

denote the error of the quadrature formula. If $p_{2}$ is a polynomial of degree $\leq 2$, a direct computation shows that $\operatorname{Err}\left(p_{2}\right)=0$. Now, let $g \in C^{3}([0,1])$. The Taylor formula of order 2 at $s=0$ reads

$$
g(s)=p_{2}(s)+\varphi(s)
$$

with $p_{2}(s)=g(0)+s g^{\prime}(0)+s^{2} g^{\prime \prime}(0) / 2$ and

$$
\varphi(s)=\frac{1}{2} \int_{0}^{s}(s-\sigma)^{2} g^{(3)}(\sigma) \mathrm{d} \sigma .
$$

Using $\operatorname{Err}\left(p_{2}\right)=0$ and the linearity of $\operatorname{Err}$, we find $\operatorname{Err}(g)=\operatorname{Err}(\varphi)$. Next, we compute $\operatorname{Err}(\varphi)$ using definition (4.16). The values of $\varphi(0)$ and $\varphi(1)$ are straightforward. On computing, we find $\varphi^{\prime \prime}(1)=\int_{0}^{1} g^{(3)}(\sigma) \mathrm{d} \sigma$. In the first term of the right-hand side of (4.16), we apply Fubini's theorem (recall $\varphi$ is defined by an integral). Summing up, we have obtained

$$
\operatorname{Err}(\varphi)=\int_{0}^{1} \frac{(1-\sigma)^{3}}{6} g^{(3)}(\sigma) \mathrm{d} \sigma-\left(\frac{1}{4} \int_{0}^{1}(1-\sigma)^{2} g^{(3)}(\sigma) \mathrm{d} \sigma-\frac{1}{12} \int_{0}^{1} g^{(3)}(\sigma) \mathrm{d} \sigma\right),
$$

i.e.

$$
\operatorname{Err}(\varphi)=\frac{1}{2} \int_{0}^{1} k_{2}^{-}(\sigma) g^{(3)}(\sigma) \mathrm{d} \sigma
$$

where $k_{2}^{-}$is defined above. The claim is proved.

We have (compare with Lemma 3.1, and notice that $w_{1}^{n+1}$ is defined by (4.28))

Lemma 4.5 (Energy estimate for any $\tau$ ). If $\left(U^{n}, V^{n}, Z^{n}, W^{n}\right)_{n \geq 1}$ is a sequence in $\left(\mathbb{R}^{N_{h}}\right)^{4}$ which complies with (4.3), then for all $n \geq 0$,

$$
\frac{\mathcal{E}_{h}\left(U^{n+1}, V^{n+1}\right)-\mathcal{E}_{h}\left(U^{n}, V^{n}\right)}{\tau}+\left|\dot{V}^{n+1 / 2}\right|_{-1}^{2} \leq v_{1}^{1 / 2} q^{n} w_{1}^{n+1}
$$

As a consequence, for all $k \geq 0$, we have

$$
\mathcal{E}_{h}\left(U^{N_{0}+k}, V^{N_{0}+k}\right)+\sum_{j=0}^{k-1} \tau\left|\dot{V}^{N_{0}+j+1 / 2}\right|_{-1}^{2} \leq \exp \left(16 c_{5} \frac{\tau|q|^{N_{0}}}{1-|q|}\left|v_{1}^{1 / 2}\right|\right)\left(\mathcal{E}_{h}\left(U^{N_{0}}, V^{N_{0}}\right)+c_{7} \frac{\tau|q|^{N_{0}}}{1-|q|}\left|v_{1}^{1 / 2}\right|\right)
$$

where $N_{0}=N_{0}\left(\beta, c_{5}, \tau,\left|v_{1}^{0}\right|\right) \in \mathbb{N}$ is such that

$$
2 c_{5} \tau|q|^{N_{0}}\left|v_{1}^{1 / 2}\right| \leq 1 / 2
$$

$c_{7}=2 c_{1} c_{5}+c_{6}$ depends only on $f, f_{+}, f_{-}$(see (2.4) and (4.1)), and $q$ is defined by (4.11).

Proof. Let $\delta u_{h}^{n}=u_{h}^{n+1}-u_{h}^{n}$. Since $f_{+}=F_{+}^{\prime}$ and $f_{-}=F_{-}^{\prime}$, we have

$$
\begin{aligned}
& F_{+}\left(u_{h}^{n+1}\right)-F_{+}\left(u_{h}^{n}\right)=\delta u_{h}^{n} \int_{0}^{1} f_{+}\left(u_{h}^{n}+s \delta u_{h}^{n}\right) \mathrm{d} s, \\
& F_{-}\left(u_{h}^{n+1}\right)-F_{-}\left(u_{h}^{n}\right)=\delta u_{h}^{n} \int_{0}^{1} f_{-}\left(u_{h}^{n}+s \delta u_{h}^{n}\right) \mathrm{d} s .
\end{aligned}
$$


Choosing $g(s)=f_{+}\left(u_{h}^{n}+s \delta u_{h}^{n}\right)$ in (4.14), we find

$$
\int_{0}^{1} f_{+}\left(u_{h}^{n}+s \delta u_{h}^{n}\right) \mathrm{d} s=\frac{1}{2}\left(f_{+}\left(u_{h}^{n}\right)+f_{+}\left(u_{h}^{n+1}\right)\right)-\frac{\left(\delta u_{h}^{n}\right)^{2}}{12} f_{+}^{\prime \prime}\left(u_{h}^{n}\right)-\frac{\left(\delta u_{h}^{n}\right)^{3}}{2} \int_{0}^{1} k_{2}^{+}(\sigma) f_{+}^{\prime \prime \prime}\left(u_{h}^{n}+\sigma \delta u_{h}^{n}\right) \mathrm{d} \sigma .
$$

Setting $g(s)=f_{-}\left(u_{h}^{n}+s \delta u_{h}^{n}\right)$ in (4.15), we find

$$
\int_{0}^{1} f_{-}\left(u_{h}^{n}+s \delta u_{h}^{n}\right) \mathrm{d} s=\frac{1}{2}\left(f_{-}\left(u_{h}^{n}\right)+f_{-}\left(u_{h}^{n+1}\right)\right)-\frac{\left(\delta u_{h}^{n}\right)^{2}}{12} f_{-}^{\prime \prime}\left(u_{h}^{n+1}\right)+\frac{\left(\delta u_{h}^{n}\right)^{3}}{2} \int_{0}^{1} k_{2}^{-}(\sigma) f_{-}^{\prime \prime \prime}\left(u_{h}^{n}+\sigma \delta u_{h}^{n}\right) \mathrm{d} \sigma .
$$

Adding (4.21) and (4.22) leads to

$$
\begin{aligned}
F\left(u_{h}^{n+1}\right)-F\left(u_{h}^{n}\right)= & \delta u_{h}^{n}\left[\frac{1}{2}\left(f\left(u_{h}^{n}\right)+f\left(u_{h}^{n+1}\right)\right)\right. \\
& \left.-\frac{\left(\delta u_{h}^{n}\right)^{2}}{12}\left(f_{+}^{\prime \prime}\left(u_{h}^{n}\right)+f_{-}^{\prime \prime}\left(u_{h}^{n+1}\right)\right)\right]-\alpha^{n},
\end{aligned}
$$

where

$$
\alpha^{n}=\frac{\left(\delta u_{h}^{n}\right)^{4}}{2}\left(\int_{0}^{1} k_{2}^{+}(\sigma) f_{+}^{\prime \prime \prime}\left(u_{h}^{n}+\sigma \delta u_{h}^{n}\right) \mathrm{d} \sigma-\int_{0}^{1} k_{2}^{-}(\sigma) f_{-}^{\prime \prime \prime}\left(u_{h}^{n}+\sigma \delta u_{h}^{n}\right) \mathrm{d} \sigma\right) \geq 0
$$

by assumption (H3) on the decomposition. Next, we choose $\xi_{h}=\delta u_{h}^{n}$ in the last equation of (4.2). This gives

$$
\left(w_{h}^{n+1}, \delta u_{h}^{n}\right)-\left(\nabla z_{h}^{n+1}, \nabla \delta u_{h}^{n}\right)+2\left(\nabla u_{h}^{n+1 / 2}, \nabla \delta u_{h}^{n}\right)=\left(F\left(u_{h}^{n+1}\right), 1\right)-\left(F\left(u_{h}^{n}\right), 1\right)+\left(\alpha^{n}, 1\right) .
$$

Using the vector form with $\delta U^{n}=U^{n+1}-U^{n}$, and eliminating $z_{h}^{n+1}$, we obtain

$$
F_{h}\left(U^{n+1}\right)-F_{h}\left(U^{n}\right)+\left(\alpha^{n}, 1\right)=\left(W^{n+1}\right)^{t} \delta U^{n}-\frac{1}{2}\left(\left|A U^{n+1}\right|^{2}-\left|A U^{n}\right|^{2}\right)+\left|A^{1 / 2} U^{n+1}\right|^{2}-\left|A^{1 / 2} U^{n}\right|^{2} .
$$

The second equation in (4.2) implies

$$
-\dot{W}^{n+1}=\dot{A}^{-1}\left(\beta \frac{\left(\dot{V}^{n+1}-\dot{V}^{n}\right)}{\tau}+\dot{V}^{n+1 / 2}\right) .
$$

Plugging this in (4.27), together with $\delta U^{n}=\tau V^{n+1 / 2}$, we get

$$
E_{h}\left(U^{n+1}\right)-E_{h}\left(U^{n}\right)+\left(\alpha^{n}, 1\right)+\frac{\beta}{2}\left(\left|\dot{V}^{n+1}\right|_{-1}^{2}-\left|\dot{V}^{n}\right|_{-1}^{2}\right)+\tau\left|\dot{V}^{n+1 / 2}\right|_{-1}^{2}=\tau v_{1}^{n+1 / 2} w_{1}^{n+1} .
$$

This yields the energy estimate (4.18).

Choosing $\xi_{h}=1$ in the last equation of (4.2), we find

$$
w_{1}^{n+1}=\frac{1}{2}\left(f\left(u_{h}^{n}\right)+f\left(u_{h}^{n+1}\right), 1\right)-\frac{1}{12}\left(\left(u_{h}^{n+1}-u_{h}^{n}\right)^{2}\left(f_{+}^{\prime \prime}\left(u_{h}^{n}\right)+f_{-}^{\prime \prime}\left(u_{h}^{n+1}\right)\right), 1\right) .
$$

Thus, by (4.1), we deduce

$$
\begin{aligned}
\left|w_{1}^{n+1}\right| & \leq\left(\frac{1}{2}\left(\left|f\left(u_{h}^{n}\right)\right|+\left|f\left(u_{h}^{n+1}\right)\right|\right)+\frac{1}{12}\left(\left(u_{h}^{n+1}-u_{h}^{n}\right)^{2}\left(\left|f_{+}^{\prime \prime}\left(u_{h}^{n}\right)\right|+\left|f_{-}^{\prime \prime}\left(u_{h}^{n+1}\right)\right|\right), 1\right)\right) \\
& \leq\left(c_{5}\left(F\left(u_{h}^{n}\right)+F\left(u_{h}^{n+1}\right)\right)+c_{6}, 1\right) .
\end{aligned}
$$

As a consequence, by (3.16), we get

$$
\begin{aligned}
\mathcal{E}_{h}\left(U^{n+1}, V^{n+1}\right)-\mathcal{E}_{h}\left(U^{n}, V^{n}\right)+\tau\left|\dot{V}^{n+1 / 2}\right|_{-1}^{2} & \leq \tau\left|v_{1}^{n+1 / 2}\right|\left(c_{5}\left(F\left(u_{h}^{n}\right), 1\right)+c_{5}\left(F\left(u_{h}^{n+1}\right), 1\right)+c_{6}\right) \\
& \leq \tau\left|v_{1}^{n+1 / 2}\right|\left(2 c_{5} E_{h}\left(U^{n+1}\right)+2 c_{5} E_{h}\left(U^{n}\right)+2 c_{5} c_{1}+c_{6}\right)
\end{aligned}
$$


Let us set

$$
\mathcal{E}_{h}^{n}=\mathcal{E}_{h}\left(U^{n}, V^{n}\right)=E_{h}\left(U^{n}\right)+\frac{\beta}{2}\left|\dot{V}^{n}\right|_{-1}^{2} \quad \text { and } \quad \mathcal{E}_{h}^{n+1}=\mathcal{E}_{h}\left(U^{n+1}, V^{n+1}\right) .
$$

So far, we have proved that

$$
\mathcal{E}_{h}^{n+1}+\tau\left|\dot{V}^{n+1 / 2}\right|_{-1}^{2} \leq \mathcal{E}_{h}^{n}+\tau|q|^{n}\left|v_{1}^{1 / 2}\right|\left(2 c_{5} \mathcal{E}_{h}^{n}+2 c_{5} \mathcal{E}_{h}^{n+1}+c_{7}\right),
$$

where $c_{7}=2 c_{5} c_{1}+c_{6}$. Let $N_{0}=N_{0}\left(\beta, c_{5}, \tau,\left|v_{1}^{0}\right|\right) \in \mathbb{N}$ satisfy (4.20). Then for $n \geq N_{0}$, we have

$$
\left(1-2 c_{5} \tau|q|^{n}\left|v_{1}^{1 / 2}\right|\right) \mathcal{E}_{h}^{n+1}+\tau\left|\dot{V}^{n+1 / 2}\right|_{-1}^{2} \leq\left(1+2 c_{5} \tau|q|^{n}\left|v_{1}^{1 / 2}\right|\right) \mathcal{E}_{h}^{n}+c_{7} \tau|q|^{n}\left|v_{1}^{1 / 2}\right| .
$$

We divide by this inequality $\left(1-2 c_{5} \tau|q|^{n}\left|v_{1}^{1 / 2}\right|\right)$ and we use that (by the mean value inequality) for all $x \in[0,1 / 2]$,

$$
1 \leq \frac{1}{1-x} \quad \text { and } \quad \frac{1+x}{1-x} \leq 1+8 x \leq \exp (8 x)
$$

We obtain

$$
\mathcal{E}_{h}^{n+1}+\tau\left|\dot{V}^{n+1 / 2}\right|_{-1}^{2} \leq \exp \left(16 c_{5} \tau|q|^{n}\left|v_{1}^{1 / 2}\right|\right)\left(\mathcal{E}_{h}^{n}+c_{7} \tau|q|^{n}\left|v_{1}^{1 / 2}\right|\right),
$$

for all $n \geq N_{0}$. By induction, for all $k \in \mathbb{N}$, we deduce

$$
\begin{aligned}
\mathcal{E}_{h}^{N_{0}+k}+\sum_{j=0}^{k-1} \tau\left|\dot{V}^{N_{0}+j+1 / 2}\right|_{-1}^{2} \leq & \exp \left(16 c_{5} \tau\left|v_{1}^{1 / 2}\right| \sum_{j=0}^{k-1}|q|^{N_{0}+j}\right) \mathcal{E}_{h}^{N_{0}} \\
& +\sum_{j=0}^{k-1} \exp \left(16 c_{5} \tau\left|v_{1}^{1 / 2}\right|\left(|q|^{N_{0}}+\cdots+|q|^{N_{0}+k-1-j}\right)\right) c_{7} \tau|q|^{N_{0}+j}\left|v_{1}^{1 / 2}\right| .
\end{aligned}
$$

Estimate (4.19) follows by using the inequality $\sum_{j=0}^{k-1}|q|^{N_{0}+j} \leq|q|^{N_{0}} /(1-|q|)$.

Theorem 4.6 (Uniqueness for small $\tau$ ). For any $\left(u_{h}^{0}, v_{h}^{0}\right) \in V_{h} \times V_{h}$, there exists $\tau^{\star}=\tau^{\star}(h)>0$ such that for any $\tau \in\left(0, \tau^{\star}\right)$, there is a unique sequence $\left(u_{h}^{n}, v_{h}^{n}, z_{h}^{n}, w_{h}^{n}\right)_{n \geq 1}$ which complies with (4.2). Moreover, $\tau^{\star}$ can be made independent of $h$ if $\left(u_{h}^{0}, v_{h}^{0}\right)_{h>0}$ is a family such that

$$
\left|\left\langle v_{h}^{0}\right\rangle\right|+\mathcal{E}_{h}\left(u_{h}^{0}, v_{h}^{0}\right) \leq C_{1},
$$

for some constant $C_{1}$ independent of $h$.

Remark 4.7. Condition (4.29) is ensured by assumptions (3.28) and (3.29).

Proof. Assume that $\left(u_{h}^{n}, v_{h}^{n}\right)$ is uniquely determined for some $n \geq 0$. We have seen that $u_{1}^{n+1}=\left\langle u_{h}^{n+1}\right\rangle$ is uniquely determined (see (4.9)). It is sufficient to show that $\dot{u}_{h}^{n+1} \simeq \dot{U}^{n+1}$ is uniquely determined by (4.10), for $\tau$ sufficiently small and independent of $n$. Then $v_{h}^{n+1}$ can be recovered by the first equation in (4.5).

Assume that (4.10) has two solutions $\dot{u}_{h}^{n+1} \simeq \dot{U}^{n+1}$ and $\underline{\dot{u}}_{h}^{n+1} \simeq \underline{\dot{U}}^{n+1}$. We subtract the two resulting systems (4.10), and we multiply by $\delta \dot{U}=\dot{U}^{n+1}-\underline{\dot{U}}^{n+1} \simeq \delta \dot{u}_{h}=\delta u_{h}$. We obtain

$\frac{2 \beta}{\tau^{2}}\left|\delta \dot{u}_{h}\right|_{-1, h}^{2}+\frac{1}{\tau}\left|\delta \dot{u}_{h}\right|_{-1, h}^{2}+\frac{1}{2}\left\|\mathcal{A}_{h} \delta \dot{u}_{h}\right\|^{2}-\left|\delta \dot{u}_{h}\right|_{1}^{2}+\frac{1}{2}\left(f\left(u_{h}^{n+1}\right)-f\left(\underline{u}_{h}^{n+1}\right), \delta u_{h}\right)-\left(g\left(u_{h}^{n}, u_{h}^{n+1}\right)-g\left(u_{h}^{n}, \underline{u}_{h}^{n+1}\right), \delta u_{h}\right)=0$.

By $(2.1), f^{\prime}$ is a polynomial of even degree with strictly positive leading coefficient, so that $f^{\prime}$ is bounded from below. There exists an (optimal) constant $c_{f} \geq 0$ such that

$$
f^{\prime}(s) \geq-c_{f} \quad \forall s \in \mathbb{R} .
$$


By the mean value theorem,

$$
\left(f\left(u_{h}^{n+1}\right)-f\left(\underline{u}_{h}^{n+1}\right), \delta u_{h}\right) \geq-c_{f}\left\|\delta \dot{u}_{h}\right\|^{2} .
$$

On the other hand, by (4.6), we have

$$
g\left(u_{h}^{n}, u_{h}^{n+1}\right)-g\left(u_{h}^{n}, \underline{u}_{h}^{n+1}\right)=\sum_{0 \leq k+l \leq 2 p+1} b_{k, l}\left(u_{h}^{n}\right)^{k}\left[\left(u_{h}^{n+1}\right)^{l}-\left(\underline{u}_{h}^{n+1}\right)^{l}\right],
$$

so that by Hölder's inequality,

$$
\left|\left(g\left(u_{h}^{n}, u_{h}^{n+1}\right)-g\left(u_{h}^{n}, \underline{u}_{h}^{n+1}\right), \delta u_{h}\right)\right| \leq C_{n}^{\prime}\left\|\delta \dot{u}_{h}\right\|_{L^{2 p+2}(\Omega)}^{2},
$$

where

$$
C_{n}^{\prime}=C^{\prime}\left(\left\|u_{h}^{n}\right\|_{L^{2 p+2}(\Omega)},\left\|u_{h}^{n+1}\right\|_{L^{2 p+2}(\Omega)},\left\|\underline{u}_{h}^{n+1}\right\|_{L^{2 p+2}(\Omega)}\right),
$$

and $C^{\prime}$ is a nondecreasing function of its arguments. Thus, by (2.3), equation (4.30) implies

$$
\frac{2 \beta}{\tau^{2}}\left|\delta \dot{u}_{h}\right|_{-1, h}^{2}+\frac{1}{\tau}\left|\delta \dot{u}_{h}\right|_{-1, h}^{2}+\frac{1}{2}\left\|\mathcal{A}_{h} \delta \dot{u}_{h}\right\|^{2} \leq \frac{c_{f}}{2}\left\|\delta \dot{u}_{h}\right\|^{2}+\left(C_{n}^{\prime} C_{S}+1\right)\left|\delta \dot{u}_{h}\right|_{1}^{2} .
$$

Let $\left(u_{h}^{0}, v_{h}^{0}\right)$ be given initial data and let $\tau^{\star}=\min \left\{2 \beta,\left(4 c_{5}\left|v_{1}^{0}\right|\right)^{-1}\right\}$. Then for $\tau \in\left(0, \tau^{\star}\right), q=(2 \beta-\tau) /(2 \beta+\tau) \in$ $(0,1)$ and $(4.20)$ is satisfied for $N_{0}=0$ since $\left|v_{1}^{1 / 2}\right|=\left|(1+q) v_{1}^{0} / 2\right| \leq\left|v_{1}^{0}\right|$. Moreover,

$$
\frac{\tau}{1-q}=\beta+\frac{\tau}{2} \leq 2 \beta
$$

By the energy estimate (4.19) and (3.25), $C_{n}^{\prime}$ is bounded by a constant independent of $n$ and $\tau$. Since all norms are equivalent in $V_{h}$, estimate (4.32) implies that for $\tau>0$ small enough (but dependent on $h$ !), $\delta u_{h}=0$.

Now, assume that the bound (4.29) is satisfied, and replace $\tau^{\star}$ by

$$
\tau^{\star}=\min \left\{2 \beta,\left(4 c_{5} C_{1}\right)^{-1}\right\} .
$$

By the energy estimate (4.19), $\mathcal{E}_{h}\left(U^{n}, V^{n}\right)$ is bounded by a constant independent of $h, n$ and $\tau$. Thus, by (3.25), $C_{n}^{\prime}$ is bounded by a constant $C^{\star}$ independent of $h$ and $n$. We apply Lemma 4.8 below with $\varepsilon_{1}=1 /\left(4\left(C^{\star} C_{S}+1\right)\right)$ and $\varepsilon_{2}=1 /\left(2 c_{f}\right)$, and we obtain

$$
\frac{2 \beta}{\tau^{2}}\left|\delta \dot{u}_{h}\right|_{-1, h}^{2}+\frac{1}{\tau}\left|\delta \dot{u}_{h}\right|_{-1, h}^{2} \leq\left(\frac{c_{f}}{2}\left(\frac{1}{4 \varepsilon_{2}^{2}}+\frac{1}{4}\right)+\frac{C^{\star} C_{S}+1}{4 \varepsilon_{1}^{2}}\right)\left|\delta \dot{u}_{h}\right|_{-1, h}^{2} .
$$

We see that for $\tau>0$ small enough (independent of $h$ now), $\delta \dot{u}_{h}=0$ and the proof is complete.

Lemma 4.8. Let $\varepsilon_{1}, \varepsilon_{2}>0$. Then, for all $\dot{u}_{h} \in \dot{V}_{h}$, there hold

$$
\begin{aligned}
\left|\dot{u}_{h}\right|_{1}^{2} & \leq \varepsilon_{1}\left\|\dot{\mathcal{A}}_{h} \dot{u}_{h}\right\|^{2}+\frac{1}{4 \varepsilon_{1}^{2}}\left|\dot{u}_{h}\right|_{-1, h}^{2}, \\
\left\|\dot{u}_{h}\right\|^{2} & \leq \varepsilon_{2}\left\|\dot{\mathcal{A}}_{h} \dot{u}_{h}\right\|^{2}+\left(\frac{1}{4 \varepsilon_{2}^{2}}+\frac{1}{4}\right)\left|\dot{u}_{h}\right|_{-1, h}^{2} .
\end{aligned}
$$

Proof. By arguing as in (3.12), we see that

$$
\left|\dot{A}^{1 / 2} \dot{U}\right|^{2}=(\dot{A} \dot{U})^{t} \dot{U} \leq \frac{\varepsilon_{1}}{2}|\dot{A} \dot{U}|^{2}+\frac{1}{2 \varepsilon_{1}}|\dot{U}|^{2} .
$$

Let $\varepsilon>0$. Similarly, we have

$$
|\dot{U}|^{2}=\left(\dot{A}^{1 / 2} \dot{U}\right)^{t} \dot{A}^{-1 / 2} \dot{U} \leq \varepsilon\left|\dot{A}^{1 / 2} \dot{U}\right|^{2}+\frac{1}{4 \varepsilon}\left|\dot{A}^{-1 / 2} \dot{U}\right|^{2} .
$$


Thus, we get

$$
\left|\dot{A}^{1 / 2} \dot{U}\right|^{2} \leq \frac{\varepsilon_{1}}{2}|\dot{A} \dot{U}|^{2}+\frac{1}{2 \varepsilon_{1}}\left(\varepsilon\left|\dot{A}^{1 / 2} \dot{U}\right|^{2}+\frac{1}{4 \varepsilon}\left|\dot{A}^{-1 / 2} \dot{U}\right|^{2}\right) .
$$

By choosing $\varepsilon=\varepsilon_{1}$, we obtain (4.33). Next, we plug (4.33) into (4.35), with $\varepsilon=1$ and $\varepsilon_{1}=\varepsilon_{2}$, and we deduce (4.34).

\subsection{Convergence as $(h, \tau) \rightarrow(0,0)$}

For a time step $\tau>0$, let $\left(u_{h}^{n}, v_{h}^{n}, z_{h}^{n}, w_{h}^{n}\right)_{n \geq 1}$ be a solution of the fully discrete scheme (4.2). We define the following functions from $\mathbb{R}_{+}$into $V_{h}$ : for $t \in[n \tau,(n+1) \tau)$ with $n \in \mathbb{N}$,

$$
\begin{aligned}
& u_{h}^{\tau}(t)=((n+1)-t / \tau) u_{h}^{n}+(t / \tau-n) u_{h}^{n+1}, \\
& \bar{u}_{h}^{\tau}(t)=u_{h}^{n+1}, \\
& \underline{u}_{h}^{\tau}(t)=u_{h}^{n}, \\
& \hat{u}_{h}^{\tau}(t)=\left(u_{h}^{n}+u_{h}^{n+1}\right) / 2 .
\end{aligned}
$$

We define similarly the functions $v_{h}^{\tau}, \bar{v}_{h}^{\tau}, \underline{v}_{h}^{\tau}, \hat{v}_{h}^{\tau}$ associated to the sequence $\left(v_{h}^{n}\right)_{n \geq 0}$ and the functions $\bar{z}_{h}^{\tau}, \bar{w}_{h}^{\tau}$. Notice that $\hat{u}_{h}^{\tau}=\left(\bar{u}_{h}^{\tau}+\underline{u}_{h}^{\tau}\right) / 2$ for all $t \in \mathbb{R}_{+}$and that

$$
\partial_{t} u_{h}^{\tau}=\left(u_{h}^{n+1}-u_{h}^{n}\right) / \tau \quad \text { in } \quad \mathcal{D}^{\prime}\left((0, \infty) ; V_{h}\right) .
$$

The convergence for the fully discrete scheme is essentially the same as for the space semi-discrete scheme. It reads:

Theorem 4.9. Let $\left(u_{0}, v_{0}\right) \in H_{\text {per }}^{2} \times H_{\text {per }}^{-1}$. Assume that $\left(u_{h}^{0}, v_{h}^{0}\right)_{h>0}$ is a family of functions in $V_{h} \times V_{h}$ which satisfies assumptions (3.28)-(3.29) as $h \rightarrow 0$. Then the solution $\left(u_{h}^{\tau}, v_{h}^{\tau}\right)$ associated to the fully discrete scheme (4.2) tends to the energy solution of problem (1.1) and (1.2) in the following sense, as $(h, \tau) \rightarrow(0,0)$ :

$$
\begin{aligned}
u_{h}^{\tau} & \rightarrow \text { u weakly } \star \text { in } L^{\infty}\left(\mathbb{R}_{+} ; H_{p e r}^{1}\right), \\
u_{h}^{\tau} & \rightarrow \text { strongly in } C\left([0, T], H_{\text {per }}^{1}\right), \text { for all } T>0, \\
\mathcal{A}_{h} u_{h}^{\tau} & \rightarrow \mathcal{A} u \text { weakly } \star \text { in } L^{\infty}\left(\mathbb{R}_{+} ; L^{2}(\Omega)\right), \\
\dot{\mathcal{A}}_{h}^{-1} \partial_{t} \dot{u}_{h}^{\tau} & \rightarrow \dot{\mathcal{A}}^{-1} \partial_{t} \dot{u} \text { weakly } \star \text { in } L^{\infty}\left(\mathbb{R}_{+} ; \dot{H}_{\text {per }}^{1}\right) \text { and weakly in } L^{2}\left(\mathbb{R}_{+} ; \dot{H}_{\text {per }}^{1}\right) .
\end{aligned}
$$

Proof. We proceed as in the proof of Theorem 3.6. The main difference is that we have to deal with the various functions associated to the sequence $\left(u_{h}^{n}, v_{h}^{n}, z_{h}^{n}, w_{h}^{n}\right)$. We first consider the conservation law for the mass. By choosing $\varphi_{h}=1$ and $\psi_{h}=1$ in (4.2), we find

$$
\left\{\begin{array}{l}
\left(\partial_{t} u_{h}^{\tau}, 1\right)=\left\langle\hat{v}_{h}^{\tau}\right\rangle \\
\beta \partial_{t}\left\langle v_{h}^{\tau}\right\rangle+\left\langle\hat{v}_{h}^{\tau}\right\rangle=0,
\end{array}\right.
$$

in $\mathcal{D}^{\prime}(0, \infty)$. The estimates below show that $\left(u_{h}^{\tau}\right)_{h>0, \tau>0}$ is bounded in $L^{\infty}\left(\mathbb{R}_{+} ; H_{p e r}^{1}\right)$, so that, up to a subsequence, $u_{h}^{\tau} \rightarrow u$ in $L^{\infty}\left(\mathbb{R}_{+} ; H_{p e r}^{1}\right)$ weakly $\star$, and so

$$
\left(\partial_{t} u_{h}^{\tau}, 1\right) \rightarrow\left(\partial_{t} u, 1\right) \quad \text { in } \mathcal{D}^{\prime}(0, \infty), \text { as }(h, \tau) \rightarrow(0,0) .
$$

By (4.12), $\left|v_{1}^{n}\right| \leq\left|v_{1}^{0}\right|$ for all $n$. Thus, $\left\langle\hat{v}_{h}^{\tau}\right\rangle$ is bounded in $L^{\infty}\left(\mathbb{R}_{+}\right)$, and so, up to a subsequence, $\left\langle\hat{v}_{h}^{\tau}\right\rangle$ converges weakly $\star$ in $L^{\infty}\left(\mathbb{R}_{+}\right)$to some function $a \in L^{\infty}\left(\mathbb{R}_{+}\right)$. Moreover, by (4.12), we have

$$
\left|v_{1}^{n+1}-v_{1}^{n}\right|=|q|^{n}|1-q|\left|v_{1}^{0}\right|=|q|^{n} \frac{2 \tau}{2 \beta+\tau}\left|v_{1}^{0}\right| \leq \frac{\tau}{\beta}\left|v_{1}^{0}\right|,
$$


Observe now that

$$
v_{h}^{\tau}-\hat{v}_{h}^{\tau}=(t / \tau-(n+1 / 2))\left(v_{h}^{n+1}-v_{h}^{n}\right), \quad t \in[n \tau,(n+1) \tau), \quad(n \in \mathbb{N}) .
$$

Therefore we get $\left|\left\langle v_{h}^{\tau}\right\rangle-\left\langle\hat{v}_{h}^{\tau}\right\rangle\right| \leq \tau\left|v_{1}^{0}\right| /(2 \beta)$ and so $\left|\left\langle v_{h}^{\tau}\right\rangle-\left\langle\hat{v}_{h}^{\tau}\right\rangle\right|$ converges uniformly to 0 in $\mathbb{R}_{+}$, as $(h, \tau) \rightarrow$ $(0,0)$. Hence $\left\langle v_{h}^{\tau}\right\rangle$ converges to $a$ weakly $\star$ in $L^{\infty}\left(\mathbb{R}_{+}\right)$. We can pass to the limit in $(4.36)$ in the sense of distributions on $(0, \infty)$ and we find

$$
\left\{\begin{array}{l}
\left(\partial_{t} u, 1\right)=a(t) \\
\beta \partial_{t} a(t)+a(t)=0
\end{array}\right.
$$

which is the conservation law for the mass.

We now turn to the energy estimate. Let

$$
\tau^{\star}=\min \left\{2 \beta,\left(4 c_{5} \sup _{h>0}\left|\left\langle v_{h}^{0}\right\rangle\right|\right)^{-1}\right\} .
$$

If $\tau \leq \tau^{\star}$, then (4.20) is satisfied for $N_{0}=0$ (and for all $h>0$ ). Since

$$
\frac{\tau}{1-q}=\beta+\tau / 2 \leq 2 \beta
$$

the energy estimate (4.19) implies

$$
\mathcal{E}_{h}\left(u_{h}^{n}, v_{h}^{n}\right)+\sum_{k=0}^{n-1} \tau\left|v_{h}^{k+1 / 2}\right|_{-1, h}^{2} \leq \exp \left(32 \beta c_{5}\left|v_{1}^{0}\right|\right)\left(\mathcal{E}_{h}\left(u_{h}^{0}, v_{h}^{0}\right)+2 \beta c_{7}\left|v_{1}^{0}\right|\right),
$$

for all $n \geq 0$. Assumptions (3.28) and (3.29) imply that $\mathcal{E}_{h}\left(u_{h}^{0}, v_{h}^{0}\right)$ and $\left|v_{1}^{0}\right|$ are bounded by a constant independent of $h$. The right-hand side of (4.38) is bounded by a constant independent of $h$ and $\tau$. Thus, by (3.25), $u_{h}^{\tau}$ is uniformly bounded in $H_{p e r}^{1}, \bar{z}_{h}^{\tau}=\mathcal{A}_{h} \hat{u}_{h}^{\tau}$ is uniformly bounded in $L^{2}(\Omega)$, and

$$
\dot{r}_{h}^{\tau}:=\dot{\mathcal{A}}_{h}^{-1} \partial_{t} \dot{u}_{h}^{\tau}=\dot{\mathcal{A}}_{h}^{-1} \dot{\hat{v}}_{h}^{\tau}
$$

is uniformly bounded in $H_{p e r}^{1}$ and bounded in $L^{2}\left(\mathbb{R}_{+} ; H_{\text {per }}^{1}\right)$. Here we have used that

$$
\int_{0}^{\infty}\left|\dot{r}_{h}^{\tau}\right|_{1}^{2} \mathrm{~d} t=\sum_{k=0}^{\infty} \tau\left|v_{h}^{k+1 / 2}\right|_{-1, h}^{2} \leq C .
$$

By arguing as in (3.32), we see that for all $0 \leq s \leq t$,

$$
\left\|\dot{u}_{h}^{\tau}(t)-\dot{u}_{h}^{\tau}(s)\right\|^{2} \leq 4\left\|\dot{r}_{h}^{\tau}\right\|_{L^{\infty}\left(\mathbb{R}_{+} ; H_{p e r}^{1}\right)}\left\|\dot{u}_{h}^{\tau}\right\|_{L^{\infty}\left(\mathbb{R}_{+} ; H_{p e r}^{1}\right)}|t-s| .
$$

Moreover, for all $0 \leq s \leq t$, observe that

$$
\left|\left\langle u_{h}^{\tau}(t)\right\rangle-\left\langle u_{h}^{\tau}(s)\right\rangle\right|=\left|\int_{s}^{t}\left\langle\hat{v}_{h}^{\tau}(\sigma)\right\rangle \mathrm{d} \sigma\right| \leq\left|\left\langle v_{h}^{0}\right\rangle\right||t-s| .
$$

Thus, for all $T>0$, there is a constant $C_{T}$ independent of $(h, \tau)$ such that

$$
\left\|u_{h}^{\tau}(t)-u_{h}^{\tau}(s)\right\| \leq C_{T}|t-s|^{1 / 2},
$$

for all $0 \leq s \leq t \leq T$. By the Ascoli-Arzelà theorem, $\left(u_{h}^{\tau}\right)$ is precompact in the space $C\left([0, T] ; L^{2}(\Omega)\right)$, for all $T>0$ [37]. Applying (4.39) with $s=n \tau$ and $t=(n+1) \tau$ yields $\left\|u_{h}^{n+1}-u_{h}^{n}\right\| \leq C_{T} \tau^{1 / 2}$, so that

$$
\left\|u_{h}^{\tau}-\bar{u}_{h}^{\tau}\right\|_{L^{\infty}\left(0, T ; L^{2}(\Omega)\right)} \rightarrow 0 \quad \text { and } \quad\left\|u_{h}^{\tau}-\underline{u}_{h}^{\tau}\right\|_{L^{\infty}\left(0, T ; L^{2}(\Omega)\right)} \rightarrow 0
$$


as $\tau \rightarrow 0$. Using Lemma 3.5, we have, for some $u, z$ and $\dot{r}$, and up to a subsequence,

$$
\begin{aligned}
u_{h}^{\tau}, \hat{u}_{h}^{\tau} & \rightarrow u \text { weakly } \star \text { in } L^{\infty}\left(\mathbb{R}_{+} ; H_{p e r}^{1}\right), \\
\bar{u}_{h}^{\tau}, \underline{u}_{h}^{\tau} & \rightarrow u \text { strongly in } L^{\infty}\left([0, T] ; L^{2}(\Omega)\right), \text { for all } T>0, \\
u_{h}^{\tau} & \rightarrow u \text { strongly in } C\left([0, T] ; H_{p e r}^{1}\right), \text { for all } T>0, \\
\bar{u}_{h}^{\tau}, \underline{u}_{h}^{\tau} & \rightarrow u \text { a.e. in } \mathbb{R}_{+} \times \Omega, \\
\bar{z}_{h}^{\tau} & \rightarrow z \text { weakly } \star \text { in } L^{\infty}\left(\mathbb{R}_{+} ; L^{2}(\Omega)\right), \\
\dot{r}_{h}^{\tau} & \rightarrow \dot{r} \text { weakly } \star \text { in } L^{\infty}\left(\mathbb{R}_{+} ; \dot{H}_{p e r}^{1}\right) \text { and weakly in } L^{2}\left(\mathbb{R}_{+} ; \dot{H}_{p e r}^{1}\right),
\end{aligned}
$$

as $(h, \tau) \rightarrow(0,0)$. It remains to show that the limit, which we have denoted $u$ for notational convenience, is the energy solution of (1.1) and (1.2).

Let $\varphi \in H_{p e r}^{1}$ and set $\varphi_{h}=\Pi_{h}(\varphi)$, so that $\varphi_{h} \rightarrow \varphi$ strongly in $H_{p e r}^{1}$. The first equation in (4.2) reads

$$
\left(\partial_{t} u_{h}^{\tau}, \varphi_{h}\right)=\left(\hat{v}_{h}^{\tau}, \varphi_{h}\right)
$$

By arguing as in (3.34) and letting $(h, \tau) \rightarrow(0,0)$, we obtain that

$$
\partial_{t}(\dot{u}, \dot{\varphi})=(\nabla \dot{r}, \nabla \dot{\varphi})=\langle\dot{v}, \dot{\varphi}\rangle_{H_{p e r}^{-1}, H_{p e r}^{1}}
$$

in $\mathcal{D}^{\prime}(0, \infty)$, with $\dot{v}=\dot{\mathcal{A}} \dot{r}$. This shows that $\partial_{t} \dot{u}=\dot{v} \in L^{\infty}\left(\mathbb{R}_{+} ; H_{p e r}^{1}\right)$.

Next, we set $\psi \in H_{\text {per }}^{2}$ and we let $\psi_{h}=\Pi_{h}(\psi)$ so that $\psi_{h} \rightarrow \psi$ strongly in $H_{\text {per }}^{1}$. We have $\psi_{h}=\sum_{i=1}^{N_{h}} \psi_{i} e_{h}^{i}$ and $\Psi=\left(\psi_{1}, \ldots, \psi_{N_{h}}\right)^{t}$ is the vector associated to $\psi_{h}$. On multiplying (4.5) by $\dot{\Psi}^{t} \dot{A}^{-1}$, we find

$$
\begin{aligned}
& \beta\left(\partial_{t} \dot{v}_{h}^{\tau}, \dot{\psi}_{h}\right)_{-1, h}+\left(\dot{\hat{v}}_{h}^{\tau}, \dot{\psi}_{h}\right)_{-1, h}+\left(\nabla \bar{z}_{h}^{\tau}, \nabla \psi_{h}\right)-2\left(\nabla \hat{u}_{h}^{\tau}, \nabla \psi_{h}\right) \\
& \left.+\frac{1}{2}\left(f\left(\underline{u}_{h}^{\tau}\right)+f\left(\bar{u}_{h}^{\tau}\right), \dot{\psi}_{h}\right)-\frac{1}{12}\left(\left(\bar{u}_{h}^{\tau}-\underline{u}_{h}^{\tau}\right)^{2}\left(f_{+}^{\prime \prime} \underline{u}_{h}^{\tau}\right)+f_{-}^{\prime \prime}\left(\bar{u}_{h}^{\tau}\right)\right), \dot{\psi}_{h}\right)=0 .
\end{aligned}
$$

By arguing as in the proof of Theorem 3.6, we get

$$
\left(\dot{\hat{v}}_{h}^{\tau}, \dot{\psi}_{h}\right)_{-1, h} \rightarrow(\dot{v}, \dot{\psi})_{-1},\left(\nabla \bar{z}_{h}^{\tau}, \nabla \psi_{h}\right) \rightarrow(z, \mathcal{A} \psi),\left(\nabla \hat{u}_{h}^{\tau}, \nabla \psi_{h}\right) \rightarrow(\nabla u, \nabla \psi)
$$

in $\mathcal{D}^{\prime}(0, \infty)$. Thanks to the Sobolev injection $H_{p e r}^{1} \hookrightarrow L^{2 p+2}(\Omega)$, for all $T>0$, the terms

$$
\left\|f\left(\underline{u}_{h}^{\tau}\right)\right\|_{L^{q}\left(0, T ; L^{q}(\Omega)\right)},\left\|f\left(\bar{u}_{h}^{\tau}\right)\right\|_{L^{q}\left(0, T ; L^{q}(\Omega)\right)},
$$

and

$$
\left\|\left(\bar{u}_{h}^{\tau}-\underline{u}_{h}^{\tau}\right)^{2}\left(f_{+}^{\prime \prime}\left(\underline{u}_{h}^{\tau}\right)+f_{-}^{\prime \prime}\left(\bar{u}_{h}^{\tau}\right)\right)\right\|_{L^{q}\left(0, T ; L^{q}(\Omega)\right)}
$$

are bounded by a constant independent of $h$ and $\tau$, for $q=(2 p+2) /(2 p+1) \in(1,2)$. We can therefore pass to the limit in the nonlinear terms, and we find that

$$
\frac{1}{2}\left(f\left(\underline{u}_{h}^{\tau}\right)+f\left(\bar{u}_{h}^{\tau}\right), \dot{\psi}_{h}\right) \rightarrow(f(u), \dot{\psi})
$$

and

$$
\frac{1}{12}\left(\left(\bar{u}_{h}^{\tau}-\underline{u}_{h}^{\tau}\right)^{2}\left(f_{+}^{\prime \prime}\left(\underline{u}_{h}^{\tau}\right)+f_{-}^{\prime \prime}\left(\bar{u}_{h}^{\tau}\right)\right), \dot{\psi}_{h}\right) \rightarrow 0
$$

in $\mathcal{D}^{\prime}(0, \infty)$. Thus, the first term in equation (4.40) has a limit in $\mathcal{D}^{\prime}(0, \infty)$,

$$
\beta\left(\partial_{t} \dot{v}_{h}^{\tau}, \dot{\psi}_{h}\right)_{-1, h} \rightarrow \eta_{\psi} .
$$


As a consequence,

$$
\left(\dot{\bar{v}}_{h}^{\tau}-\underline{\dot{v}}_{h}^{\tau}, \dot{\psi}_{h}\right)_{-1, h}=\tau\left(\partial_{t} \dot{v}_{h}^{\tau}, \dot{\psi}_{h}\right)_{-1, h} \rightarrow 0
$$

in $\mathcal{D}^{\prime}(0, \infty)$. Thus, by $(4.37)$ and $(4.41)$, we have

$$
\left(\dot{v}_{h}^{\tau}, \dot{\psi}_{h}\right)_{-1, h} \rightarrow(\dot{v}, \dot{\psi})_{-1}
$$

in $\mathcal{D}^{\prime}(0, \infty)$. Summing up, we have proved that

$$
\beta \partial_{t}(\dot{v}, \dot{\psi})_{-1}+(\dot{v}, \dot{\psi})_{-1}+(z, \mathcal{A} \psi)-2(\nabla u, \nabla \psi)+(f(u), \dot{\psi})=0
$$

in $\mathcal{D}^{\prime}(0, \infty)$, with $v=\partial_{t} u$ and $z=\mathcal{A} u$. We conclude as in Theorem 3.6 that $\left(u, u_{t}\right)$ is the energy solution of $(1.1)$ and (1.2). Note that the whole family converges to $\left(u, u_{t}\right)$ due to the uniqueness of the limit.

Remark 4.10. Assume that $f$ is a more general nonlinearity satisfying

$$
f \in C^{3}(\mathbb{R}), f(0)=0,
$$

and consider the decomposition

(H3') $F=F_{+}+F_{-}$, where $F_{+}$and $F_{-}$are functions in $C^{4}(\mathbb{R})$ such that $F_{+}^{(4)} \geq 0, F_{-}^{(4)} \leq 0$.

We denote $f_{+}=F_{+}^{\prime}$ and $f_{-}=F_{-}^{\prime}$. If $d=1$ and $\left\langle v_{0}\right\rangle=0$, then taking advantage of the Sobolev injection $H_{\text {per }}^{1} \subset L^{\infty}(\Omega)$, it is easily seen that (4.18) holds, i.e. the scheme is unconditionally stable. Proposition 4.2 also holds, i.e. the scheme has second-order consistency error. These two features of the Gomez and Hughes scheme have been proved in several situations (see [18-20]), assuming enough regularity on the solution.

In the general case, in order for the results of Section 4 to hold (second order consistency error, solvability for any time step, unique solvability for small time steps, energy estimate and convergence as $(h, \tau) \rightarrow(0,0))$, other assumptions are needed, in addition to (H3'). For instance, we can take $f$ satisfying (4.42), (H3'), (2.15), (3.37) to $(3.39)$ and $f_{+}$and $f_{-}$satisfying

$$
\begin{array}{r}
\left|f_{+}^{\prime \prime \prime}(s)\right| \leq \alpha_{11}|s|^{2 p-2}+\alpha_{12}, \\
\left|f_{-}^{\prime \prime \prime}(s)\right| \leq \alpha_{13}|s|^{2 p-2}+\alpha_{14}, \\
\left|f_{-}^{\prime \prime}(s)\right| \leq \alpha_{15}|s|^{2 p-1-\gamma}+\alpha_{16}
\end{array}
$$

for all $s \in \mathbb{R}$, where $\alpha_{11}, \ldots, \alpha_{16}$ are positive constants, $\gamma \in(0,1]$, and $p \in[1, \infty)(p \in[1,2]$ if $d=3)$ is the same (real) number as in (3.37) and (3.39). The last assumption (4.45) is used only in the proof of existence; the parameter $\gamma$ is needed to replace the assumption on the degrees made in (H3). We note that the physically relevant nonlinearity $f(s)=s^{3}+(1-\varepsilon) s$ with $\varepsilon \in \mathbb{R}$ and the choice $f_{+}=f, f_{-}=0$ satisfies all these conditions for $p=1$ and $\gamma=1$.

\section{Convergence to Equilibrium}

In this section, we prove that any solution of the fully discrete scheme converges to a single equilibrium, for any time step $\tau>0$. The parameter $h$ is fixed (so that assumption (H2) is not relevant). We adapt the proof from [23] in a discrete setting. The main idea is to use the gradient-like flow structure of the problem and a suitable Łojasiewicz inequality. In three space dimensions, in addition to (H1) and (H3), we need the following assumption:

(H4) If $d=3$, then either $V_{h} \subset L^{\infty}(\Omega)$ or $p=1$. 
Theorem 5.1. Let $\tau>0$ denote the time step and let $\left(U^{n}, V^{n}\right)_{n \geq 0}$ denote any sequence in $\mathbb{R}^{N_{h}} \times \mathbb{R}^{N_{h}}$ which complies with $(4.5)$. Then $\left(U^{n}, V^{n}\right)$ converges to $\left(U^{\infty}, 0\right)$, where $U^{\infty}=\left(u_{1}^{\infty}, \dot{U}^{\infty}\right)$ is a stationary solution with average constraint, i.e.,

$$
\left\{\begin{array}{l}
u_{1}^{\infty}=M=u_{1}^{0}+\beta v_{1}^{0}, \\
\dot{A}^{2} \dot{U}^{\infty}-2 \dot{A} \dot{U}^{\infty}+\dot{\nabla} F_{h}\left(U^{\infty}\right)=0 .
\end{array}\right.
$$

We first prove the following.

Lemma 5.2. Let the assumptions of Theorem 5.1 hold. Then $V^{n+1 / 2} \rightarrow 0$.

Proof. Since $|q|<1$, estimate (4.20) is satisfied for $N_{0}$ large enough. By the energy estimate (4.19), $\sum_{n=N_{0}}^{\infty}\left|\dot{V}^{n+1 / 2}\right|_{-1}^{2}<\infty$. In particular, $\dot{V}^{n+1 / 2} \rightarrow 0$ in $\mathbb{R}^{N_{h}-1}$. Moreover, $v_{1}^{n+1 / 2}=q^{n} v_{1}^{1 / 2}$ by (4.12), so $V^{n+1 / 2} \rightarrow 0$, as claimed.

For any $M \in \mathbb{R}$, we introduce the auxiliary function $F_{M}(y)=F(M+y)$ and the following functionals

$$
\begin{aligned}
F_{M, h}(\dot{U}) & =\left(F_{M}\left(\dot{u}_{h}\right), 1\right), \\
E_{M, h}(\dot{U}) & =\frac{1}{2}|\dot{A} \dot{U}|^{2}-\left|\dot{A}^{1 / 2} \dot{U}\right|^{2}+F_{M, h}(\dot{U}), \\
\mathcal{E}_{M, h}(\dot{U}, \dot{V}) & =E_{M, h}(\dot{U})+\frac{\beta}{2}|\dot{V}|_{-1}^{2},
\end{aligned}
$$

defined for every $\dot{U} \simeq \dot{u}_{h}$ and every $\dot{V}$ in $\mathbb{R}^{N_{h}-1}$.

For any $M \in \mathbb{R}$, we also consider

$$
\mathfrak{S}_{M}=\left\{U \in \mathbb{R}^{N_{h}}: U \text { satisfies }(5.1)\right\} .
$$

For any sequence $\left(U^{n}, V^{n}\right)_{n \geq 0}$ in $\mathbb{R}^{N_{h}} \times \mathbb{R}^{N_{h}}$, we define its $\omega$-limit set in $\mathbb{R}^{N_{h}} \times \mathbb{R}^{N_{h}}$ :

$$
\omega\left(\left(U^{n}, V^{n}\right)_{n \geq 0}\right)=\left\{\left(U^{\star}, V^{\star}\right): \exists n_{j} \nearrow \infty,\left(U^{n_{j}}, V^{n_{j}}\right) \rightarrow\left(U^{\star}, V^{\star}\right) \mid\right\} .
$$

Similarly, we set

$$
\omega\left(\left(U^{n}\right)_{n \geq 0}\right)=\left\{U^{\star}: \exists n_{j} \nearrow \infty, U^{n_{j}} \rightarrow U^{\star}\right\}
$$

We have

Proposition 5.3. Let the assumptions of Theorem 5.1 hold. Then $\omega\left(\left(U^{n}, V^{n}\right)_{n \geq 0}\right)$ is a nonempty compact and connected set such that

$$
\omega\left(\left(U^{n}, V^{n}\right)_{n \geq 0}\right)=\omega\left(\left(U^{n}\right)_{n \geq 0}\right) \times\{0\} \subset\left\{\left(U^{\star}, 0\right): U^{\star} \in \mathfrak{S}_{M}\right\},
$$

with $M=u_{1}^{0}+\beta v_{1}^{0}$. Moreover, $E_{M, h}$ is constant on $\omega\left(\left(U^{n}\right)_{n}\right)$.

This result implies in particular that $V^{n} \rightarrow 0$, as proved below.

Proof. Since $q=(2 \beta-\tau) /(2 \beta+\tau)$, we can rewrite (4.13) as

$$
u_{1}^{n}=u_{1}^{0}+\left(1-q^{n}\right) \beta v_{1}^{0} .
$$

Let $M=u_{1}^{0}+\beta v_{1}^{0}$. We introduce the auxiliary functions

$$
f_{M}(y)=f(M+y) \quad \text { and } \quad \hat{f}_{M}(r, s)=\hat{f}(M+r, M+s),
$$


where

$$
\hat{f}(r, s)=\frac{1}{2}(f(r)+f(s))-\frac{1}{12}(s-r)^{2}\left(f_{+}^{\prime \prime}(r)+f_{-}^{\prime \prime}(s)\right) .
$$

We also set

$$
F_{M, \pm}(y)=F_{ \pm}(M+y) \quad \text { and } f_{M, \pm}(y)=f_{ \pm}(M+y),
$$

so that $F_{M}=F_{M,+}+F_{M,-}$ and $F_{M,+}^{(4)} \geq 0, F_{M,-}^{(4)} \leq 0$. In particular, the function $F_{M}$ satisfies the decomposition (H3), and we have

$$
\hat{f}_{M}(r, s)=\frac{1}{2}\left(f_{M}(r)+f_{M}(s)\right)-\frac{1}{12}(s-r)^{2}\left(f_{M,+}^{\prime \prime}(r)+f_{M,-}^{\prime \prime}(s)\right) .
$$

Then we rewrite the second equation in (4.5) in the following form

$\beta\left(\dot{V}^{n+1}-\dot{V}^{n}\right) / \tau+\dot{V}^{n+1 / 2}+\dot{A}\left(\dot{A}^{2} \dot{U}^{n+1 / 2}-2 \dot{A} \dot{U}^{n+1 / 2}+\dot{J}_{M}\left(\dot{U}^{n}, \dot{U}^{n+1}\right)\right)=\dot{A}\left(\dot{J}_{M}\left(\dot{U}^{n}, \dot{U}^{n+1}\right)-\dot{J}\left(U^{n}, U^{n+1}\right)\right)$,

where

$$
\begin{aligned}
\dot{J}_{M}\left(\dot{U}^{n}, \dot{U}^{n+1}\right) & =\left(\left(\hat{f}_{M}\left(\dot{u}_{h}^{n}, \dot{u}_{h}^{n+1}\right), e_{i}\right)\right)_{2 \leq i \leq N_{h}}, \\
\dot{J}\left(U^{n}, U^{n+1}\right) & =\left(\left(\hat{f}\left(u_{h}^{n}, u_{h}^{n+1}\right), e_{i}\right)\right)_{2 \leq i \leq N_{h}} .
\end{aligned}
$$

Multiplying (5.6) by $\left(\dot{U}^{n+1}-\dot{U}^{n}\right)^{t} \dot{A}^{-1}$, using that (4.5) implies

$$
\dot{U}^{n+1}-\dot{U}^{n}=\tau \dot{V}^{n+1 / 2},
$$

we find

$$
\begin{aligned}
& \frac{\beta}{2}\left(\left|\dot{V}^{n+1}\right|_{-1}^{2}-\left|\dot{V}^{n}\right|_{-1}^{2}\right)+\tau\left|\dot{V}^{n+1 / 2}\right|_{-1}^{2}+\frac{1}{2}\left(\left|\dot{A} \dot{U}^{n+1}\right|^{2}-\left|\dot{A} \dot{U}^{n}\right|^{2}\right) \\
& -\left(\left|\dot{A}^{1 / 2} \dot{U}^{n+1}\right|^{2}-\left|\dot{A}^{1 / 2} \dot{U}^{n}\right|^{2}\right)+\left\langle\dot{J}_{M}\left(\dot{U}^{n}, \dot{U}^{n+1}\right), \dot{U}^{n+1}-\dot{U}^{n}\right\rangle \\
& =\left(\hat{f}_{M}\left(\dot{u}_{h}^{n}, \dot{u}_{h}^{n+1}\right)-\hat{f}\left(u_{h}^{n}, u_{h}^{n+1}\right), \dot{u}_{h}^{n+1}-\dot{u}_{h}^{n}\right) .
\end{aligned}
$$

Using (4.26) with $F$ replaced by $F_{M}$ and $u_{h}^{n}, u_{h}^{n+1}$ replaced by $\dot{u}_{h}^{n}, \dot{u}_{h}^{n+1}$, we obtain

$$
\left(F_{M}\left(\dot{u}_{h}^{n+1}\right), 1\right)-\left(F_{M}\left(\dot{u}_{h}^{n}\right), 1\right) \leq\left\langle\dot{J}_{M}\left(\dot{U}^{n}, \dot{U}^{n+1}\right), \dot{U}^{n+1}-\dot{U}^{n}\right\rangle .
$$

By (5.5), for any solution $u_{h}^{n} \simeq U^{n}$ of (4.5), we have

$$
\hat{f}\left(u_{h}^{n}, u_{h}^{n+1}\right)=\hat{f}_{M}\left(\dot{u}_{h}^{n}-\beta q^{n} v_{1}^{0}, \dot{u}_{h}^{n+1}-\beta q^{n+1} v_{1}^{0}\right) .
$$

Thus we get

$$
\begin{aligned}
& \left(\hat{f}_{M}\left(\dot{u}_{h}^{n}, \dot{u}_{h}^{n+1}\right)-\hat{f}\left(u_{h}^{n}, u_{h}^{n+1}\right), \dot{u}_{h}^{n+1}-\dot{u}_{h}^{n}\right) \\
& =\left(\hat{f}_{M}\left(\dot{u}_{h}^{n}, \dot{u}_{h}^{n+1}\right)-\hat{f}_{M}\left(\dot{u}_{h}^{n}-\beta q^{n} v_{1}^{0}, \dot{u}_{h}^{n+1}-\beta q^{n+1} v_{1}^{0}\right), \dot{u}_{h}^{n+1}-\dot{u}_{h}^{n}\right) \\
& =-\beta q^{n} v_{1}^{0}\left(\int_{0}^{1} \partial_{r} \hat{f}_{M}\left(\tilde{u}_{h}^{n}(\sigma), \tilde{u}_{h}^{n+1}(\sigma)\right)+q \partial_{s} \hat{f}_{M}\left(\tilde{u}_{h}^{n}(\sigma), \tilde{u}_{h}^{n+1}(\sigma)\right) \mathrm{d} s, \dot{u}_{h}^{n+1}-\dot{u}_{h}^{n}\right)
\end{aligned}
$$

where $\tilde{u}_{h}^{n}(\sigma)=\dot{u}_{h}^{n}-\sigma \beta q^{n} v_{1}^{0}$ for $\sigma \in[0,1]$. By the energy estimate (4.19) and (3.25), the sequence $\left(u_{h}^{n}\right)_{n \geq 0}$ is bounded in $H_{p e r}^{1}$. Moreover, the function $\hat{f}_{M}$ is a polynomial in $(r, s)$ of total degree equal to $2 p+1$. Using the Sobolev injection $H_{p e r}^{1} \hookrightarrow L^{2 p+2}(\Omega)$, we obtain

$$
\begin{aligned}
\left|\left(\hat{f}_{M}\left(\dot{u}_{h}^{n}, \dot{u}_{h}^{n+1}\right)-\hat{f}\left(u_{h}^{n}, u_{h}^{n+1}\right), \dot{u}_{h}^{n+1}-\dot{u}_{h}^{n}\right)\right| & \leq \beta|q|^{n}\left|v_{1}^{0}\right| C\left(\left\|u_{h}^{n}\right\|_{1},\left\|u_{h}^{n+1}\right\|_{1}\right)\left\|\dot{u}_{h}^{n+1}-\dot{u}_{h}^{n}\right\|_{1} \\
& \leq \frac{1}{4 \tau}\left|\dot{U}^{n+1}-\dot{U}^{n}\right|_{-1}^{2}+C_{0}|q|^{2 n} .
\end{aligned}
$$


Here and in the following, $C_{k}(k=0,1, \ldots)$ denotes a constant independent of $n$ (but which may depend on $\tau, h$ and other parameters of the problem). In the last inequality we have used that all norms are equivalent in $\dot{V}_{h}$. Adding up (5.8), (5.9) and (5.11), we find

$$
\mathcal{E}_{M, h}\left(\dot{U}^{n+1}, \dot{V}^{n+1}\right)-\mathcal{E}_{M, h}\left(\dot{U}^{n}, \dot{V}^{n}\right)+\frac{3 \tau}{4}\left|\dot{V}^{n+1 / 2}\right|_{-1}^{2} \leq C_{0}|q|^{2 n}
$$

for all $n \geq 0$.

Set now

$$
\mathcal{G}^{n}=\left\langle\dot{A}^{-1} \dot{V}^{n}, \dot{A}^{-1}\left(\dot{A}^{2} \dot{U}^{n}-2 \dot{A} \dot{U}^{n}+\dot{\nabla} F_{M, h}\left(\dot{U}^{n}\right)\right\rangle_{-1},\right.
$$

where $\langle\dot{U}, \dot{V}\rangle_{-1}=\dot{U}^{t} \dot{A}^{-1} \dot{V}$, for all $\dot{U}, \dot{V} \in \mathbb{R}^{N_{h}-1}$. We have $\mathcal{G}^{n}=\delta \mathcal{G}_{1}^{n}+\delta \mathcal{G}_{2}^{n}$, where

$$
\begin{aligned}
& \delta \mathcal{G}_{1}^{n}=\left\langle\dot{A}^{-1}\left(\dot{V}^{n+1}-\dot{V}^{n}\right), \dot{A}^{-1}\left(\dot{A}^{2} \dot{U}^{n}-2 \dot{A} \dot{U}^{n}+\dot{\nabla} F_{M, h}\left(\dot{U}^{n}\right)\right\rangle_{-1},\right. \\
& \delta \mathcal{G}_{2}^{n}=\left\langle\dot{A}^{-1} \dot{V}^{n+1}, \dot{A}^{-1}\left(\left(\dot{A}^{2}-2 \dot{A}\right)\left(\dot{U}^{n+1}-\dot{U}^{n}\right)+\dot{\nabla} F_{M, h}\left(\dot{U}^{n+1}\right)-\dot{\nabla} F_{M, h}\left(\dot{U}^{n}\right)\right)\right\rangle_{-1} .
\end{aligned}
$$

By (5.6), we have

$$
\dot{V}^{n+1}-\dot{V}^{n}=-\frac{\tau}{\beta}\left(\dot{V}^{n+1 / 2}+\dot{A} \dot{S}^{n}-\dot{A}\left(\dot{J}_{M}\left(\dot{U}^{n}, \dot{U}^{n+1}\right)-\dot{J}\left(U^{n}, U^{n+1}\right)\right)\right),
$$

where

$$
\dot{S}^{n}=\dot{A}^{2} \dot{U}^{n+1 / 2}-2 \dot{A} \dot{U}^{n+1 / 2}+\dot{J}_{M}\left(\dot{U}^{n}, \dot{U}^{n+1}\right) .
$$

Using $\dot{U}^{n}=\dot{U}^{n+1 / 2}-\left(\dot{U}^{n+1}-\dot{U}^{n}\right) / 2$ and $\dot{\nabla} F_{M, h}\left(\dot{U}^{n}\right)=\dot{J}_{M}\left(\dot{U}^{n}, \dot{U}^{n}\right)$, we find that

$$
\dot{A}^{2} \dot{U}^{n}-2 \dot{A} \dot{U}^{n}+\dot{\nabla} F_{M, h}\left(\dot{U}^{n}\right)=\dot{S}^{n}-\frac{1}{2} \dot{T}_{1}^{n}
$$

where

$$
\dot{T}_{1}^{n}=\left(\dot{A}^{2}-2 \dot{A}\right)\left(\dot{U}^{n+1}-\dot{U}^{n}\right)+2 \dot{J}_{M}\left(\dot{U}^{n}, \dot{U}^{n+1}\right)-2 \dot{J}_{M}\left(\dot{U}^{n}, \dot{U}^{n}\right) .
$$

Plugging (5.14) in the left part of $\delta \mathcal{G}_{1}^{n}$ and (5.15) in the right part, we obtain

$$
\begin{aligned}
\delta \mathcal{G}_{1}^{n}= & -\frac{\tau}{\beta}\left\langle\dot{A}^{-1} \dot{V}^{n+1 / 2}+\dot{S}^{n}-\left(\dot{J}_{M}\left(\dot{U}^{n}, \dot{U}^{n+1}\right)-\dot{J}\left(U^{n}, U^{n+1}\right)\right), \dot{A}^{-1} \dot{S}^{n}\right\rangle_{-1} \\
& +\frac{\tau}{2 \beta}\left\langle\dot{A}^{-1} \dot{V}^{n+1 / 2}+\dot{S}^{n}-\left(\dot{J}_{M}\left(\dot{U}^{n}, \dot{U}^{n+1}\right)-\dot{J}\left(U^{n}, U^{n+1}\right)\right), \dot{A}^{-1} \dot{T}_{1}^{n}\right\rangle_{-1}
\end{aligned}
$$

On expanding $\delta \mathcal{G}_{1}^{n}, \dot{T}_{1}^{n}$ and using (5.7), we find ( $\dot{I}$ is the identity matrix)

$$
\begin{aligned}
\delta \mathcal{G}_{1}^{n}+\frac{\tau}{\beta}\left|\dot{A}^{-1} \dot{S}^{n}\right|^{2}= & -\frac{\tau}{\beta}\left\langle\dot{A}^{-1} \dot{V}^{n+1 / 2}, \dot{A}^{-1} \dot{S}^{n}\right\rangle_{-1}+\frac{\tau}{\beta}\left\langle\left(\dot{J}_{M}\left(\dot{U}^{n}, \dot{U}^{n+1}\right)-\dot{J}\left(U^{n}, U^{n+1}\right)\right), \dot{A}^{-1} \dot{S}^{n}\right\rangle_{-1}, \\
& +\frac{\tau^{2}}{2 \beta}\left\langle\dot{A}^{-1} \dot{V}^{n+1 / 2},(\dot{A}-2 \dot{I}) \dot{V}^{n+1 / 2}\right\rangle_{-1} \\
& +\frac{\tau}{\beta}\left\langle\dot{A}^{-1} \dot{V}^{n+1 / 2}, \dot{A}^{-1}\left(\dot{J}_{M}\left(\dot{U}^{n}, \dot{U}^{n+1}\right)-\dot{J}_{M}\left(\dot{U}^{n}, \dot{U}^{n}\right)\right)\right\rangle_{-1} \\
& +\frac{\tau^{2}}{2 \beta}\left\langle\dot{S}^{n},(\dot{A}-2 \dot{I}) \dot{V}^{n+1 / 2}\right\rangle_{-1}+\frac{\tau}{\beta}\left\langle\dot{S}^{n},\left(\dot{J}_{M}\left(\dot{U}^{n}, \dot{U}^{n+1}\right)-\dot{J}_{M}\left(\dot{U}^{n}, \dot{U}^{n}\right)\right)\right\rangle_{-1} \\
& -\frac{\tau^{2}}{2 \beta}\left\langle\left(\dot{J}_{M}\left(\dot{U}^{n}, \dot{U}^{n+1}\right)-\dot{J}\left(U^{n}, U^{n+1}\right)\right),(\dot{A}-2 \dot{I}) \dot{V}^{n+1 / 2}\right\rangle_{-1} \\
& -\frac{\tau}{\beta}\left\langle\left(\dot{J}_{M}\left(\dot{U}^{n}, \dot{U}^{n+1}\right)-\dot{J}\left(U^{n}, U^{n+1}\right)\right),\left(\dot{J}_{M}\left(\dot{U}^{n}, \dot{U}^{n+1}\right)-\dot{J}_{M}\left(\dot{U}^{n}, \dot{U}^{n}\right)\right)\right\rangle_{-1} .
\end{aligned}
$$


Using the Cauchy-Schwarz inequality, the fact that all norms are equivalent in $\dot{V}_{h}$ and Young's inequality, we deduce

$$
\begin{aligned}
\delta \mathcal{G}_{1}^{n}+\frac{3 \tau}{4 \beta}\left|\dot{A}^{-1} \dot{S}^{n}\right|^{2} \leq & C_{1} \tau\left|\dot{V}^{n+1 / 2}\right|_{-1}^{2}+C_{2}\left|\dot{J}_{M}\left(\dot{U}^{n}, \dot{U}^{n+1}\right)-\dot{J}\left(U^{n}, U^{n+1}\right)\right|^{2} \\
& +C_{3}\left|\dot{J}_{M}\left(\dot{U}^{n}, \dot{U}^{n+1}\right)-\dot{J}_{M}\left(\dot{U}^{n}, \dot{U}^{n}\right)\right|^{2}
\end{aligned}
$$

By Bessel's inequality, we get

$$
\left|\dot{J}_{M}\left(\dot{U}^{n}, \dot{U}^{n+1}\right)-\dot{J}\left(U^{n}, U^{n+1}\right)\right|^{2} \leq\left\|\hat{f}_{M}\left(\dot{u}_{h}^{n}, \dot{u}_{h}^{n+1}\right)-\hat{f}\left(u_{h}^{n}, u_{h}^{n+1}\right)\right\|^{2} .
$$

Arguing as in (5.10), and using assumption (H4), we find that

$$
\left|\dot{J}_{M}\left(\dot{U}^{n}, \dot{U}^{n+1}\right)-\dot{J}\left(U^{n}, U^{n+1}\right)\right|^{2} \leq C_{4}|q|^{2 n}
$$

By Bessel's inequality again, we get

$$
\left|\dot{J}_{M}\left(\dot{U}^{n}, \dot{U}^{n+1}\right)-\dot{J}_{M}\left(\dot{U}^{n}, \dot{U}^{n}\right)\right|^{2} \leq\left\|\hat{f}_{M}\left(\dot{u}_{h}^{n}, \dot{u}_{h}^{n+1}\right)-\hat{f}_{M}\left(\dot{u}_{h}^{n}, \dot{u}_{h}^{n}\right)\right\|^{2} .
$$

We have

$$
\left\|\hat{f}_{M}\left(\dot{u}_{h}^{n}, \dot{u}_{h}^{n+1}\right)-\hat{f}_{M}\left(\dot{u}_{h}^{n}, \dot{u}_{h}^{n}\right)\right\|^{2}=\left\|\int_{0}^{1} \partial_{s} \hat{f}_{M}\left(\dot{u}_{h}^{n}, \dot{u}_{h}^{n}+\sigma \delta \dot{u}_{h}^{n}\right) \delta \dot{u}_{h}^{n}\right\|^{2},
$$

with $\delta \dot{u}_{h}^{n}=\dot{u}_{h}^{n+1}-\dot{u}_{h}^{n}$. The polynomial $\partial_{s} \hat{f}_{M}$ has total degree less than or equal to $2 p$. Using (H4), Hölder's inequality, (5.7), and the fact that all norms are equivalent on $V_{h}$, we get

$$
\left|\dot{J}_{M}\left(\dot{U}^{n}, \dot{U}^{n+1}\right)-\dot{J}_{M}\left(\dot{U}^{n}, \dot{U}^{n}\right)\right|^{2} \leq C_{5} \tau\left|\dot{V}^{n+1 / 2}\right|_{-1}^{2}
$$

$\left(C_{5}\right.$ depends on $\left.\tau\right)$. Summing up, we have proved

$$
\delta \mathcal{G}_{1}^{n}+\frac{3 \tau}{4 \beta}\left|\dot{A}^{-1} \dot{S}^{n}\right|^{2} \leq\left(C_{1}+C_{3} C_{5}\right) \tau\left|\dot{V}^{n+1 / 2}\right|_{-1}^{2}+C_{2} C_{4}|q|^{2 n}
$$

for all $n \geq 0$. We now consider the term $\delta \mathcal{G}_{2}^{n}$. Using $V^{n+1}=V^{n+1 / 2}+\left(V^{n+1}-V^{n}\right) / 2$, equation (5.6), and arguing as for $\delta G_{1}^{n}$, we obtain

$$
\delta \mathcal{G}_{2}^{n} \leq \frac{\tau}{4 \beta}\left|\dot{A}^{-1} \dot{S}^{n}\right|^{2}+C_{6} \tau\left|\dot{V}^{n+1 / 2}\right|_{-1}^{2}+C_{7}|q|^{2 n} .
$$

Thus, we get

$$
\mathcal{G}^{n+1}-\mathcal{G}^{n}+\frac{\tau}{2 \beta}\left|\dot{A}^{-1} \dot{S}^{n}\right|^{2} \leq C_{8} \tau\left|\dot{V}^{n+1 / 2}\right|_{-1}^{2}+C_{9}|q|^{2 n},
$$

for all $n \geq 0$, with $C_{8}=C_{1}+C_{3} C_{5}+C_{6}$ and $C_{9}=C_{2} C_{4}+C_{7}$.

Let us introduce the sequence

$$
W^{n}=2 \mathcal{E}_{M, h}\left(\dot{U}^{n}, \dot{V}^{n}\right)+\nu \mathcal{G}^{n},
$$

where $\nu>0$ is sufficiently small so that $\nu C_{8} \leq 1 / 2$. From estimates (5.12) and (5.19), it follows that

$$
W^{n+1}-W^{n}+\tau\left|\dot{V}^{n+1 / 2}\right|_{-1}^{2}+\frac{\nu \tau}{2 \beta}\left|\dot{A}^{-1} \dot{S}^{n}\right|^{2} \leq C_{10}|q|^{2 n}
$$


with $C_{10}=2 C_{0}+\nu C_{9}$. By the energy estimate (4.19), the sequence $\left(U^{n}, V^{n}\right)$ is bounded, so $\left(W^{n}\right)_{n \geq 0}$ is bounded. This implies that $W^{n}$ converges to some real number $W^{\infty}$ as $n$ tends to $\infty$. Indeed, let

$$
\widetilde{W}^{n}=W^{n}+\frac{C_{10}}{1-|q|^{2}}|q|^{2 n}
$$

Using (5.20), we see that $\widetilde{W}^{n+1}-\widetilde{W}^{n} \leq 0$, i.e. $\widetilde{W}^{n}$ is nonincreasing and since $\widetilde{W}^{n}$ is bounded, $\widetilde{W}^{n}$ has a limit $\widetilde{W}^{\infty}=W^{\infty}$.

Adding up estimate (5.20), we obtain that $\sum_{n=0}^{\infty}\left|\dot{A}^{-1} \dot{S}^{n}\right|^{2}<\infty$. In particular, $\dot{S}^{n} \rightarrow 0$. Moreover, by (5.16), we have

$$
\dot{J}_{M}\left(\dot{U}^{n}, \dot{U}^{n+1}\right)-\dot{J}\left(U^{n}, U^{n+1}\right) \rightarrow 0
$$

as $n \rightarrow \infty$. From (5.6) and Lemma 5.2, it follows that $\dot{V}^{n+1}-\dot{V}^{n} \rightarrow 0$ and that

$$
\dot{V}^{n}=\dot{V}^{n+1 / 2}-\left(\dot{V}^{n+1}-\dot{V}^{n}\right) / 2 \rightarrow 0 .
$$

If $\left(\dot{U}^{n^{\prime}}\right)_{n^{\prime}}$ is a subsequence which converges to some $\dot{U}^{\star}$, then $\dot{U}^{n^{\prime}+1} \rightarrow \dot{U}^{\star}$ as well, since $\dot{U}^{n^{\prime}+1}-\dot{U}^{n^{\prime}}=\tau \dot{V}^{n^{\prime}+1 / 2}$. Since $\dot{S}^{n^{\prime}} \rightarrow 0$ and $\dot{J}_{M}$ is continuous at $\left(\dot{U}^{\star}, \dot{U}^{\star}\right)$ with $\dot{J}_{M}\left(\dot{U}^{\star}, \dot{U}^{\star}\right)=\nabla F_{M, h}\left(\dot{U}^{\star}\right)$, we obtain that

$$
\dot{A}^{2} \dot{U}^{\star}-2 \dot{A} \dot{U}^{\star}+\dot{\nabla} F_{M, h}\left(\dot{U}^{\star}\right)=0 .
$$

Using the conservation law (5.5), we see that $U^{\star} \in \mathfrak{S}_{M}$. Finally, the sequence $\left(U^{n}, V^{n}\right)$ is bounded, and we have seen that $U^{n+1}-U^{n} \rightarrow 0, V^{n} \rightarrow 0$ so the $\omega$-limit set $\omega\left(\left(U^{n}, V^{n}\right)_{n \geq 0}\right)$ is a nonempty compact and connected subset of $\mathfrak{S}_{M} \times\{0\}$, equal to $\omega\left(\left(U^{n}\right)_{n}\right) \times\{0\}$.

Since $\dot{V}^{n} \rightarrow 0$ we have $\mathcal{G}^{n} \rightarrow 0$ (recall (5.13)), and since $W^{n} \rightarrow W^{\infty}$, we have

$$
\mathcal{E}_{M, h}\left(\dot{U}^{n}, \dot{V}^{n}\right)=(1 / 2)\left(W^{n}-\nu \mathcal{G}^{n}\right) \rightarrow W^{\infty} / 2 .
$$

By definition, $E_{M, h}\left(\dot{U}^{n}\right)=\mathcal{E}_{M, h}\left(\dot{U}^{n}, \dot{V}^{n}\right)-\frac{\beta}{2}\left|\dot{V}^{n}\right|_{-1}^{2}$, so $E_{M, h}\left(\dot{U}^{n}\right) \rightarrow W^{\infty} / 2$. This implies that $E_{M, h}$ is constant and equal to $\mathrm{e}^{\infty}:=W^{\infty} / 2$ on $\omega\left(\left(U^{n}\right)_{n}\right)$. The proof of Proposition 5.3 is complete.

We notice that the functional $E_{M, h}$ is a polynomial of the variables $\left(u_{2}, \ldots, u_{N_{h}}\right)$ of total degree $2 p+2$, so the following Łojasiewciz inequality holds:

Lemma 5.4 (Eojasiewicz inequality [32]). Let $\dot{U}^{\star} \in \mathbb{R}^{N_{h}-1}$ be a critical point of $E_{M, h}$. Then there exist constants $\theta \in(0,1 / 2)$ and $\delta>0$ such that for any $\dot{U} \in \mathbb{R}^{N_{h}-1}$ satisfying $\left|\dot{U}-\dot{U}^{\star}\right|<\delta$, there holds

$$
\left|E_{M, h}(\dot{U})-E_{M, h}\left(\dot{U}^{\star}\right)\right|^{1-\theta} \leq\left|\dot{A}^{2} \dot{U}-2 \dot{A} \dot{U}+\dot{\nabla} F_{M, h}(\dot{U})\right| .
$$

Proof of Theorem 5.1. Let $M=u_{1}^{0}+\beta v_{1}^{0}$ as previously. By Lemma 5.4 and Proposition 5.3, for every $U^{\infty} \in$ $\omega\left(\left(U^{n}\right)_{n}\right)$, there exist some $\delta>0$ and $\theta \in(0,1 / 2)$ that may depend on $\dot{U}^{\infty}$ such that the inequality $(5.21)$ holds for all $\dot{U}$ in

$$
\mathbf{B}_{\delta}\left(\dot{U}^{\infty}\right)=\left\{\dot{U} \in \mathbb{R}^{N_{h}-1}:\left|\dot{U}-\dot{U}^{\infty}\right|<\delta\right\}
$$

and $\left|E_{M, h}(\dot{U})-E_{M, h}\left(\dot{U}^{\infty}\right)\right| \leq 1$. The union of balls $\left\{\mathbf{B}_{\delta}\left(\dot{U}^{\infty}\right): \dot{U}^{\infty} \in \omega\left(\left(\dot{U}^{n}\right)_{n}\right)\right\}$ forms an open covering of $\omega\left(\left(\dot{U}^{n}\right)_{n}\right)$. Due to the compactness of $\omega\left(\left(\dot{U}^{n}\right)_{n}\right)$ in $\mathbb{R}^{N_{h}-1}$, we can find a finite sub-covering $\left\{\mathbf{B}_{\delta_{i}}\left(\dot{U}_{i}^{\infty}\right)\right\}_{i=1,2, \ldots, m}$, where the constants $\delta_{i}, \theta_{i}$ corresponding to $\dot{U}_{i}^{\infty}$ in Lemma 5.4 are indexed by $i$.

From the definition of $\omega\left(\left(\dot{U}^{n}\right)_{n}\right)$, we know that there exists a sufficiently large $n_{0}$ such that $\dot{U}^{n} \in \mathcal{U}:=$ $\cup_{i=1}^{m} \mathbf{B}_{\delta_{i}}\left(\dot{U}_{i}^{\infty}\right)$ for $n \geq n_{0}$. Taking $\theta=\min _{i=1}^{m}\left\{\theta_{i}\right\} \in(0,1 / 2)$, we infer from Lemma 5.4 that, for all $n \geq n_{0}$,

$$
\left|E_{M, h}\left(\dot{U}^{n}\right)-\mathrm{e}^{\infty}\right|^{1-\theta} \leq\left|\dot{A}^{2} \dot{U}^{n}-2 \dot{A} \dot{U}^{n}+\dot{\nabla} F_{M, h}\left(\dot{U}^{n}\right)\right|,
$$

where $\mathrm{e}^{\infty}=W^{\infty} / 2$ is the constant value of $E_{M, h}$ on $\omega\left(\left(\dot{U}^{n}\right)_{n}\right)$. 
Let us now set

$$
a_{n}=\left(\frac{\tau}{2}\left|\dot{V}^{n+1 / 2}\right|_{-1}^{2}+\frac{\nu \tau}{4 \beta}\left|\dot{A}^{-1} \dot{S}^{n}\right|^{2}\right)^{1 / 2}+|q|^{n}
$$

From (5.20), it follows that

$$
\sum_{k=n}^{\infty} a_{k}^{2} \leq W^{n}-W^{\infty}+C_{11}|q|^{2 n} .
$$

On the other hand, using the Łojasiewicz inequality (5.22) and the fact $1 /(1-\theta)<2$, we deduce that, for all $n \geq n_{0}$ (changing $n_{0}$ into a larger integer if necessary),

$$
\begin{aligned}
\left|W^{n}-W^{\infty}\right| \leq & 2\left|E_{M, h}\left(\dot{U}^{n}\right)-\mathrm{e}^{\infty}\right|+\beta\left|\dot{V}^{n}\right|_{-1}^{2}+\nu\left|\mathcal{G}^{n}\right| \\
\leq & 2\left|\dot{A}^{2} \dot{U}^{n}-2 \dot{A} \dot{U}^{n}+\dot{\nabla} F_{M, h}\left(\dot{U}^{n}\right)\right|^{1 /(1-\theta)}+\beta\left|\dot{V}^{n}\right|_{-1}^{2} \\
& +C_{12}\left|\dot{V}^{n}\right|_{-1}\left|\dot{A}^{2} \dot{U}^{n}-2 \dot{A} \dot{U}^{n}+\dot{\nabla} F_{M, h}\left(\dot{U}^{n}\right)\right| \\
\leq & C_{13}\left(\left|\dot{A}^{2} \dot{U}^{n}-2 \dot{A} \dot{U}^{n}+\dot{\nabla} F_{M, h}\left(\dot{U}^{n}\right)\right|^{1 /(1-\theta)}+\left|\dot{V}^{n}\right|_{-1}^{1 /(1-\theta)}\right) .
\end{aligned}
$$

Using $\dot{V}^{n}=\dot{V}^{n+1 / 2}-\left(\dot{V}^{n+1}-\dot{V}^{n}\right) / 2$, we deduce from (5.6) and (5.16) that

$$
\left|\dot{V}^{n}\right|_{-1} \leq C_{13}\left(\left|\dot{V}^{n+1 / 2}\right|_{-1}+\left|\dot{A}^{-1} \dot{S}^{n}\right|+|q|^{n}\right) .
$$

Similarly, from

$$
\begin{aligned}
\dot{A}^{2} \dot{U}^{n}-2 \dot{A} \dot{U}^{n}+\dot{\nabla} F_{M, h}\left(\dot{U}^{n}\right)= & \dot{S}^{n}+\left(\dot{A}^{2}-2 \dot{A}\right)\left(\dot{U}^{n}-\dot{U}^{n+1 / 2}\right) \\
& +\dot{J}_{M}\left(\dot{U}^{n}, \dot{U}^{n}\right)-\dot{J}_{M}\left(\dot{U}^{n}, \dot{U}^{n+1}\right),
\end{aligned}
$$

$\dot{U}^{n+1}-\dot{U}^{n}=\tau \dot{V}^{n+1 / 2}$ and (5.18), we infer that

$$
\left|\dot{A}^{2} \dot{U}^{n}-2 \dot{A} \dot{U}^{n}+\dot{\nabla} F_{M, h}\left(\dot{U}^{n}\right)\right| \leq C_{14}\left(\left|\dot{A}^{-1} \dot{S}^{n}\right|+\left|\dot{V}^{n+1 / 2}\right|_{-1}\right) .
$$

Collecting (5.24), (5.25) and (5.26), we obtain

$$
\left|W^{n}-W^{\infty}\right| \leq C_{15}\left(\left|\dot{V}^{n+1 / 2}\right|_{-1}^{1 /(1-\theta)}+\left|\dot{A}^{-1} \dot{S}^{n}\right|^{1 /(1-\theta)}+\left(|q|^{n}\right)^{1 /(1-\theta)}\right),
$$

for all $n \geq n_{0}$. This gives

$$
\sum_{k=n}^{\infty} a_{k}^{2} \leq C_{16} a_{n}^{1 /(1-\theta)}, \quad \forall n \geq n_{0} .
$$

From Lemma 5.5 below, we conclude that $\sum_{n=0}^{\infty} a_{n}<\infty$. In particular, we have

$$
\tau \sum_{n=0}^{\infty}\left|\dot{V}^{n+1 / 2}\right|_{-1}=\sum_{n=0}^{\infty}\left|\dot{U}^{n+1}-\dot{U}^{n}\right|<\infty .
$$

This shows that the whole sequence $\left(\dot{U}^{n}\right)_{n}$ has a limit $\dot{U}^{\infty}$ as $n \rightarrow \infty$. From (5.5), we know that $u_{1}^{n} \rightarrow M$. Thus, $\left(U^{n}\right)_{n}$ tends to some $U^{\infty}$ in $\mathbb{R}^{N_{h}}$, and the proof is complete.

For the following lemma and its proof, we adapt Lemma 4.1 in [30] in a discrete setting (see also Lem. 7.1 in [14]). 
Lemma 5.5. Let $0<\theta<1 / 2$. Assume that $\left(a_{n}\right)_{n \geq 0}$ is a sequence of nonnegative real numbers such that $\sum_{n=0}^{\infty} a_{n}^{2}<\infty$, and there are a constant $C>0$ and an integer $n_{0}$ such that

$$
\sum_{k=n}^{\infty} a_{k}^{2} \leq C a_{n}^{1 /(1-\theta)} \text { for all } n \geq n_{0} .
$$

Then $\sum_{n=0}^{\infty} a_{n}<\infty$.

Proof. First replacing $a_{n}$ by $\max \left\{a_{n}, 1\right\}$ for $0 \leq n<n_{0}$, and then taking $C$ large enough to ensure $C \geq \sum_{n=0}^{\infty} a_{n}^{2}$, we observe that (5.27) becomes valid for all $n \geq 0$. So we may assume $n_{0}=0$. Set now

$$
\rho_{n}:=\sum_{k=n}^{\infty} a_{k}^{2} \quad \text { and } \quad \sigma_{n}=\sum_{k=0}^{n} a_{k} \quad \text { for } n \geq 0 .
$$

Given any $n \geq 0$, we first raise inequality (5.27) to the power $1-\theta>0$,

$$
\rho_{n}^{1-\theta} \leq C^{1-\theta} a_{n}
$$

Next, we sum this relation and we obtain

$$
\sum_{k=0}^{n} \rho_{k}^{1-\theta} \leq C_{1} \sum_{k=0}^{n} a_{k}=C_{1} \sigma_{n}
$$

We now apply a discrete integration-by-parts on the left-hand side

$$
\sum_{k=0}^{n}[(k+2)-(k+1)] \rho_{k}^{1-\theta}=(n+2) \rho_{n}^{1-\theta}-\rho_{0}^{1-\theta}+\sum_{k=1}^{n}(k+1)\left(\rho_{k-1}^{1-\theta}-\rho_{k}^{1-\theta}\right) .
$$

Next, we notice that

$$
\rho_{k-1}^{1-\theta}-\rho_{k}^{1-\theta}=\int_{\rho_{k}}^{\rho_{k-1}}(1-\theta) s^{-\theta} \mathrm{d} s \geq(1-\theta) a_{k-1}^{2} \rho_{k-1}^{-\theta},
$$

since $\rho_{k-1}=\rho_{k}+a_{k-1}^{2}$. This gives

$$
(n+2) \rho_{n}^{1-\theta}-\rho_{0}^{1-\theta}+(1-\theta) \sum_{k=1}^{n}(k+1) a_{k-1}^{2} \rho_{k-1}^{-\theta} \leq C_{1} \sigma_{n} .
$$

It follows that, for every $n \geq 0$,

$$
(n+1) \rho_{n}^{1-\theta} \leq C_{2}\left(1+\sigma_{n}\right)
$$

and

$$
\sum_{k=1}^{n}(k+1) a_{k-1}^{2} \rho_{k-1}^{-\theta} \leq C_{2}\left(1+\sigma_{n}\right)
$$

where $C_{2}>0$ is a constant independent of $n$. Since the sequence $\left(\sigma_{n}\right)$ is nondecreasing, the former estimate yields

$$
\rho_{k-1} \leq C_{3}\left(1+\sigma_{n}\right)^{1 /(1-\theta)} k^{-1 /(1-\theta)}, \quad 1 \leq k \leq n<\infty,
$$

which we insert into the latter one, thus arriving at

$$
\sum_{k=1}^{n} k^{1+\theta /(1-\theta)} a_{k-1}^{2} \leq C_{4}\left(1+\sigma_{n}\right)^{1+\theta /(1-\theta)} .
$$


The constants $C_{3}, C_{4}$ are independent of $n \geq 0$. As a consequence, using the Cauchy-Schwarz inequality, we obtain for $n \geq 1$,

$$
\begin{aligned}
\sigma_{n-1}=\sum_{k=1}^{n} a_{k-1} & \leq\left(\sum_{k=1}^{n} k^{1 /(1-\theta)} a_{k-1}^{2}\right)^{1 / 2}\left(\sum_{k=1}^{n} k^{-1 /(1-\theta)}\right)^{1 / 2} \\
& \leq C_{5}\left(1+\sigma_{n}\right)^{1 / 2(1-\theta)}
\end{aligned}
$$

We conclude that the sequence $\left(\sigma_{n}\right)_{n}$ must be bounded, since $2(1-\theta)>1$. Indeed, assume by contradiction that $\left(\sigma_{n}\right)_{n}$ is unbounded, and set $r_{n}=1+\sigma_{n} \geq 1$. Then $\left(r_{n}\right)$ is nondecreasing, $r_{n} \rightarrow \infty$ and

$$
r_{n-1} \leq C_{6} r_{n}^{1 / 2(1-\theta)},
$$

so that $r_{n-1} / r_{n} \rightarrow 0$, and we deduce that $r_{n} \rightarrow 0$. This is the contradiction. Thus $\left(\sigma_{n}\right)$ is bounded, i.e. $\sum_{n=0}^{\infty} a_{n}<\infty$, as claimed.

Remark 5.6. In the proof of convergence to equilibrium, we have used the fact that all norms are equivalent in $V_{h}$. An interesting open question would be to prove a similar result for the time semi-discrete version of our problem.

Remark 5.7. By arguing as in the continuous case (see [23]), using the energy estimate (cf. Lem. 3.1) and the Lojasiewicz inequality 5.21, it is possible to prove that any solution $\left(u_{h}, v_{h}\right)$ of the space semi-discrete scheme (3.1) converges to a single equilibrium, provided assumptions (H1) and (H4) hold.

Remark 5.8. Assume that (H1) and (H3') are valid, for a nonlinearity $f$ which satisfies the assumptions made in Remark 4.10. Also that (H4) is replaced by

(H4') The inclusion $V_{h} \subset L^{\infty}(\Omega)$ holds $(d=1,2$ or 3$)$.

If furthermore $f$ is real analytic, then Theorem 5.1 is also true. Note that $f_{+}$and $f_{-}$do not need to be real analytic.

\section{NumericAl RESUltS}

We present some numerical results in one space dimension (obtained with the Scilab software ${ }^{3}$ and in two space dimensions (obtained with the Freefem++ software [28]). In every case, the nonlinearity $f$ is given by (1.3) for some parameter $\varepsilon$, and we set $f_{+}=f, f_{-}=0$ in assumption (H3). The space $V_{h}$ is the space of piecewise linear $\left(P^{1}\right)$ finite elements on a fixed grid. The nonlinear system at each step is solved by a Newton algorithm.

\subsection{Simulations in one space dimension}

We first choose an interval $\Omega=(0, L)$ with $L=4 \pi$. In Table 1 , we compute the error in the $C^{0}\left([0, T] ; L^{2}(\Omega)\right)$ norm (which appears in Thm. 4.9). The parameters are $\varepsilon=0.5, \beta=0.5$ and $T=2$. We use a uniform grid with space stepsize $h=L / M$ and time stepsize $\tau=T / N$. The initial value $\left(u_{h}^{0}, v_{h}^{0}\right)$ is the $P^{1}$-interpolate of $u_{0}(x)=\cos (x)+0.3 \cos (3 x), v_{0}(x)=0.1$.

For the error of the time discretization, $h=L / 160$ is fixed. Since the exact solution $u_{h}$ of the space semidiscrete scheme (3.1) and (3.2) is unknown, we use instead the solution on a fine grid with stepsize $\tau_{\text {sol }}=T / 5120$. Table 1 (left) shows the error

$$
\operatorname{err}_{h}(\tau)=\max _{0 \leq k \leq 5120}\left\|u_{h}^{\tau}\left(t_{k}\right)-u_{h}^{\tau_{s o l}}\left(t_{k}\right)\right\|_{L^{2}(0, L)}
$$

\footnotetext{
${ }^{3}$ Scilab is freely available at http://www.scilab.org/.
} 
TABLE 1. Convergence error for the time (left) and for the space (right) discretization.

\begin{tabular}{ccc}
\hline$N=T / \tau$ & $\operatorname{err}_{h}(\tau)$ & Ratio \\
\hline 80 & 0.5018208 & - \\
160 & 0.1455507 & 3.45 \\
320 & 0.0368516 & 3.95 \\
640 & 0.0091325 & 4.04 \\
1280 & 0.0022163 & 4.12 \\
\hline
\end{tabular}

\begin{tabular}{ccc}
\hline$M=L / h$ & $\operatorname{err}^{\tau}(h)$ & Ratio \\
\hline 40 & 0.7770682 & - \\
80 & 0.2735932 & 2.84 \\
160 & 0.0706798 & 3.87 \\
320 & 0.0175677 & 4.02 \\
640 & 0.0041882 & 4.20 \\
\hline
\end{tabular}
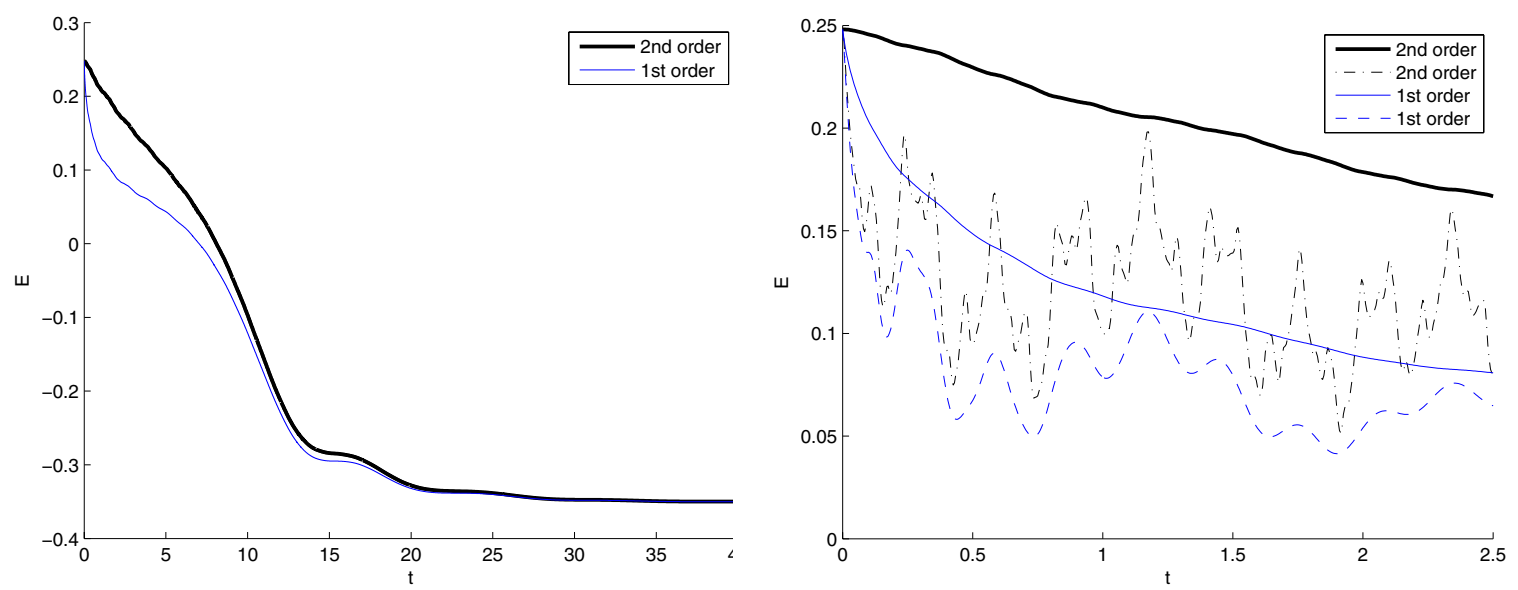

Figure 1. (Pseudo-)energy versus time, case $\left\langle v_{0}\right\rangle=0$.

evaluated on the fine grid $t_{k}=k \tau_{\text {sol }}(k=0,1, \ldots, 5120)$, and the ratio

$$
\operatorname{err}_{h}(\tau) / \operatorname{err}_{h}(\tau / 2)
$$

of consecutive errors. The computed ratio is close to 4 , which means that the convergence error for the time discretization is $O\left(\tau^{2}\right)$, as expected.

For the error of the space discretization, $\tau=T / 160$ is fixed. We use again the solution $u_{h_{s o l}}^{\tau}$ on a fine grid with stepsize $h_{\text {sol }}=L / 2560$ for the comparison. Table 1 (right) shows the error

$$
\operatorname{err}^{\tau}(h)=\max _{0 \leq k \leq 160}\left\|u_{h}^{\tau}\left(t_{k}\right)-u_{h_{s o l}}^{\tau}\left(t_{k}\right)\right\|_{L^{2}(0, L)}
$$

evaluated on the grid $t_{k}=k \tau(k=0,1, \ldots, 160)$ and the ratio $\operatorname{err}^{\tau}(h) / \operatorname{err}^{\tau}(h / 2)$ of consecutive errors. Again, the computed ratio is close to 4: the convergence error for the space discretization is $O\left(h^{2}\right)$, as expected.

Figure 1 shows the plot of the pseudo-energy $\mathcal{E}_{h}\left(u_{h}^{n}, v_{h}^{n}\right)$ (see (3.22)) versus the time $t$ (in solid line). The domain is $\Omega=(0, L)$ with $L=4 \pi$. The parameters are $\varepsilon=0.5, \beta=5, h=L / 320$ and $\tau=0.005$. The initial condition is the $P^{1}$-interpolate of $u_{0}(x)=0.1 /(1+0.7 \cos (x)), v_{0}=0$. The black color corresponds to the second-order scheme (4.2); the blue color corresponds to a first-order scheme obtained by applying to the space semi-discrete scheme (3.1) the time discretization proposed by Wang and Wise [41,42]. Both schemes are unconditionally stable.

The left figure shows the pseudo-energy on the interval $[0,40]$. Since $\left\langle v_{0}\right\rangle=0$, the pseudo-energy is nonincreasing in both cases (see (4.18)). In both cases, the evolution is driven to a stationary state, as predicted by the theory (see Thm. 5.1). We notice that the first-order scheme has a smoothing effect which creates more dissipation, especially at the beginning of the evolution. This is seen in the right figure which shows the energy 

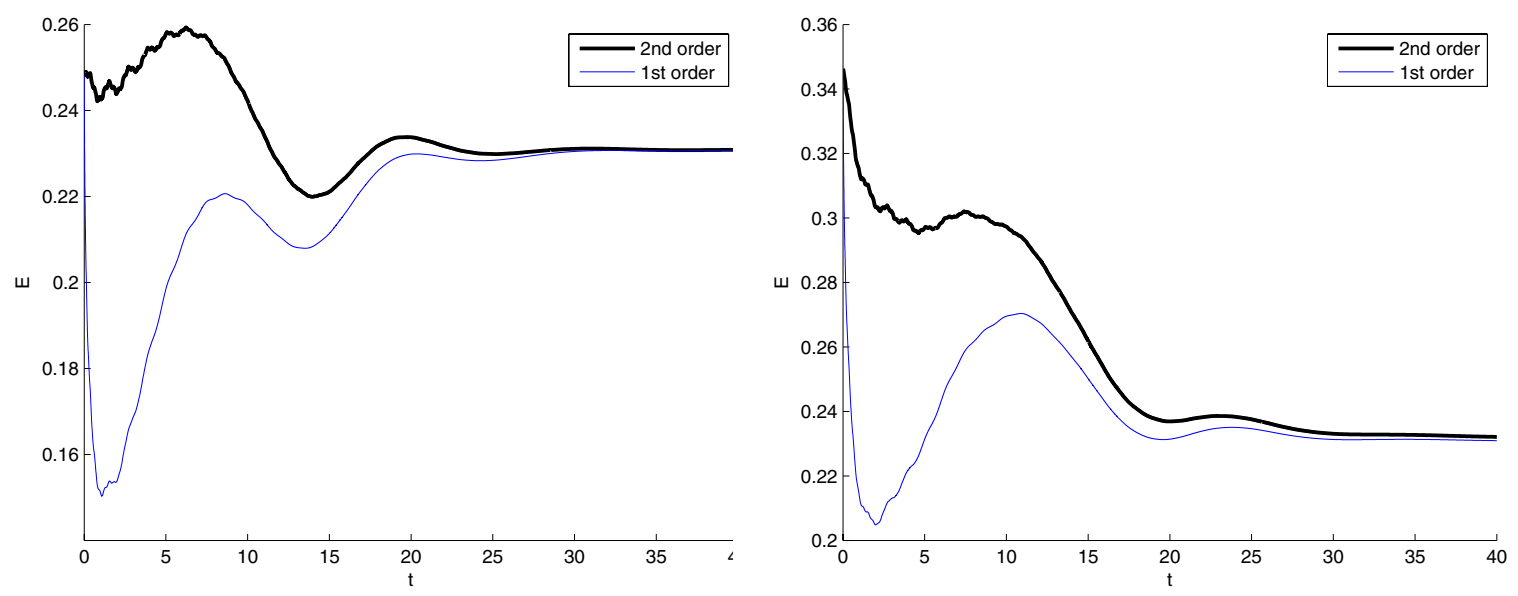

Figure 2. Pseudo-energy versus time, case $\left\langle v_{0}\right\rangle=0.034$.
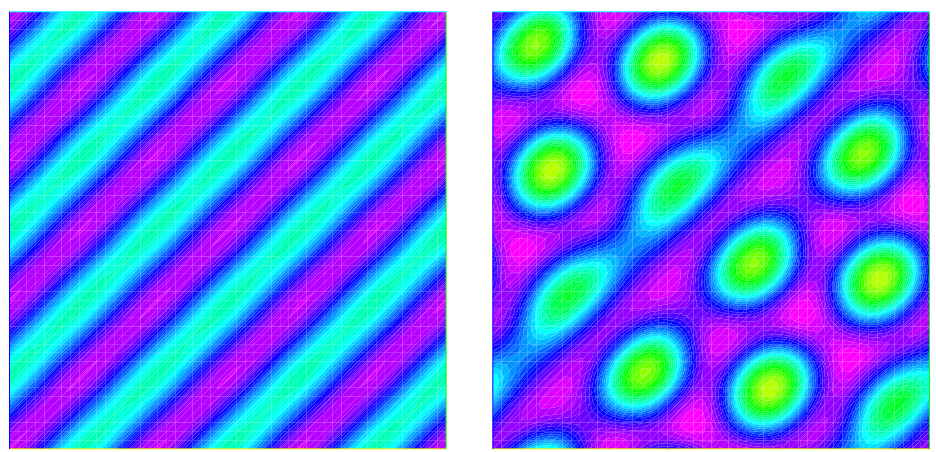

Figure 3. $t=5$ (left) and $t=26.25$ (right).

$E_{h}\left(u_{h}^{n}\right)$ on the interval $[0,2.5]$ (in dashed and dashdot), in addition to the pseudo-energy $\mathcal{E}_{h}\left(u_{h}, v_{h}\right)$ (in solid line). The difference $\mathcal{E}_{h}\left(u_{h}^{n}, v_{h}^{n}\right)-E_{h}\left(u_{h}^{n}\right)=(\beta / 2)\left|\dot{v}_{h}^{n}\right|_{-1, h}^{2}$ can be interpreted as a "kinetic energy".

Figure 2 illustrates the influence of the initial condition $v_{0}$ on the dynamics. The parameters are exactly the same as in Figure 1 (left), except for $v_{0}$. In the left part of Figure 2, $v_{0}=0.034$ is chosen constant, and in the right part, $v_{0}=0.034+\dot{v}_{0}$, where $\dot{v}_{0}$ is a random function in $V_{h}$ with zero mean value and amplitude 1 . In both cases, the pseudo-energy exhibits oscillations due to the fact that $\left\langle v_{0}\right\rangle \neq 0$. By Theorem 5.1, the sequence $\left(u_{h}^{n}\right)_{n}$ generated by the second-order algorithm converges (as $n \rightarrow \infty)$ to a steady state with mean value equal to $\left\langle u_{h}^{0}\right\rangle+\beta\left\langle v_{h}^{0}\right\rangle$. It is also seen that in the left and right parts of Figure 2, the pseudo-energy of the limiting value is close to 0.23 . These are strong indications that the two steady states corresponding to the two parts of Figure 2 are related to one another (see also [35]). Heuristically, the solution of the continuous problem converges to a global minimizer, which is uniquely defined by its mean value, up to translation invariance.

\subsection{Simulations in two space dimensions}

The domain $\Omega$ is the square $(0,6 \pi) \times(0,6 \pi)$. It is decomposed in $50 \times 50$ squares, and each square is divided along the lower left/upper right diagonal, resulting in a uniform triangulation of $\Omega$. The parameters are $\beta=0.1$, $\varepsilon=2$ and the time step is $\tau=0.25$. The initial condition is the $P^{1}$-interpolate of $u_{0}(x, y)=0.2+0.2 \cos (x) \cos (y)$ and $v_{0}=0$. Figures 3 and 4 show the evolution from stripes to a triangular distribution of drops. Numerical tests up to time $t=1250$ indicate that the triangular distribution of drops is the steady state for this simulation. 

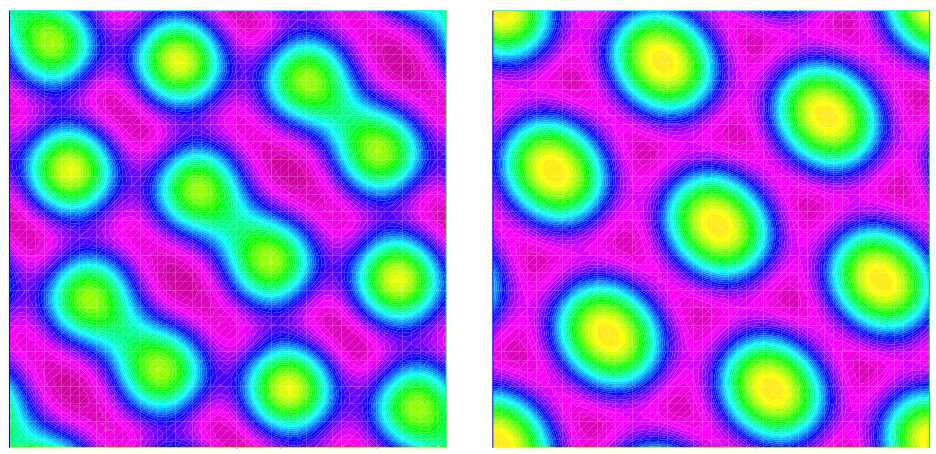

FiguRE $4 . \quad t=27.75$ (left) and $t=50$ (right).

For the continuous problem (1.1), using the translation invariance, from a triangular distribution of drops we easily build a two dimensional continuum of steady states. For the fully discrete scheme (4.2), the translation invariance is broken by the space discretization, but we expect a large number of steady states. This simulation illustrates the convergence to equilibrium result (Thm. 5.1) in a situation where the steady state is not unique.

Acknowledgements. Both authors thank the two anonymous referees whose accurate comments and questions helped improve the quality of the paper.

\section{REFERENCES}

[1] A. Abourou Ella and A. Rougirel, Steady state bifurcations for phase field crystal equations with underlying two dimensional kernel. Electron. J. Qual. Theory Differ. Equ. 60 (2015) 1-36.

[2] N.E. Alaa and M. Pierre, Convergence to equilibrium for discretized gradient-like systems with analytic features. IMA $J$. Numer. Anal. 33 (2013) 1291-1321.

[3] R. Backofen, A. Rätz and A. Voigt, Nucleation and growth by a phase field crystal (PFC) model. Philos. Mag. Lett. 87 (2007) $813-820$.

[4] A. Baskaran, Z. Hu, J. S. Lowengrub, C. Wang, S.M. Wise and P. Zhou, Energy stable and efficient finite-difference nonlinear multigrid schemes for the modified phase field crystal equation. J. Comput. Phys. 250 (2013) 270-292.

[5] A. Baskaran, J. S. Lowengrub, C. Wang and S.M. Wise, Convergence analysis of a second order convex splitting scheme for the modified phase field crystal equation. SIAM J. Numer. Anal. 51 (2013) 2851-2873.

[6] J. Bolte, A. Daniilidis, O. Ley and L. Mazet, Characterizations of Łojasiewicz inequalities: subgradient flows, talweg, convexity. Trans. Amer. Math. Soc. 362 (2010) 3319-3363.

[7] M. Cheng and J. A. Warren, An efficient algorithm for solving the phase field crystal model. J. Comput. Phys. 228 (2009) $5323-5339$.

[8] K.R. Elder and M. Grant, Modeling elastic and plastic deformations in nonequilibrium processing using phase field crystal. Phys. Rev. E 90 (2004) 051605.

[9] K.R. Elder, M. Katakowski, M. Haataja and M. Grant, Modeling elasticity in crystal growth. Phys. Rev. Lett. 88 (2002) 245701.

[10] C.M. Elliott, The Cahn-Hilliard model for the kinetics of phase separation, in Mathematical models for phase change problems (Òbidos 1988). Birkhäuser, Basel (1989) 35-73.

[11] M. Elsey and B. Wirth, A simple and efficient scheme for phase field crystal simulation. ESAIM: M2AN 47 (2013) $1413-1432$.

[12] H. Emmerich, L. Gránásy and H. Löven, Selected issues of phase-field crystal simulations. Eur. Phys. J. Plus 126 (2011) 102.

[13] H. Emmerich, H. Löwen, R. Wittkowskib, T. Gruhna, G. I. Tóth, G. Tegze and L. Gránásy, Phase field-crystal models for condensed matter dynamics on atomic length and diffusive time scales: an overview. Adv. Phys. 61 (2012) 665-743.

[14] E. Feireisl and F. Simondon, Convergence for semilinear degenerate parabolic equations in several space dimensions. J. Dyn. Differ. Equ. 12 (2000) 647-673.

[15] P. Galenko and K. Elder, Marginal stability analysis of the phase field crystal model in one spatial dimension. Phys. Rev. B 83 (2011) 064113.

[16] P. Galenko and D. Jou, Diffuse-interface model for rapid phase transformations in nonequilibrium systems. Phys. Rev. E $\mathbf{7 1}$ (2005) 046125.

[17] P. Galenko, D. Danilov and V. Lebedev, Phase-field-crystal and Swift-Hohenberg equations with fast dynamics. Phys. Rev. E 79 (2009) 051110. 
[18] P.K. Galenko, H. Gomez, N.V. Kropotin and K.R. Elder, Unconditionally stable and numerical solution of the hyperbolic phase-field crystal equation. Phys. Rev. E 88 (2013) 013310.

[19] H. Gomez and T.J.R. Hughes, Provably unconditionally stable, second-order time-accurate, mixed variational methods for phase-field models. J. Comput. Phys. 230 (2011) 5310-5327.

[20] H. Gomez and X. Nogueira, An unconditionally energy-stable method for the phase field crystal equation. Comput. Methods Appl. Mech. Engrg. 249/252 (2012) 52-61.

[21] M. Grasselli and M. Pierre, A splitting method for the Cahn-Hilliard equation with inertial term. Math. Models Methods Appl. Sci. 20 (2010) 1-28.

[22] M. Grasselli and M. Pierre, Convergence to equilibrium of solutions of the backward Euler scheme for asymptotically autonomous second-order gradient-like systems. Commun. Pure Appl. Anal. 11 (2012) 2393-2416.

[23] M. Grasselli and H. Wu, Well-posedness and longtime behavior for the modified phase-field crystal equation. Math. Models Methods Appl. Sci. 24 (2014) 2743-2783.

[24] M. Grasselli and H. Wu, Robust exponential attractors for the modified phase-field crystal equation. Discrete Contin. Dyn. Syst. Ser. A 35 (2015) 2539-2564.

[25] M. Grasselli, G. Schimperna, A. Segatti and S. Zelik, On the 3D Cahn-Hilliard equation with inertial term. J. Evol. Equ. 9 (2009) 371-404.

[26] M. Grasselli, G. Schimperna and S. Zelik, On the 2D Cahn-Hilliard equation with inertial term. Comm. Partial Differential Equ. 34 (2009) 137-170.

[27] M. Grasselli, G. Schimperna and S. Zelik, Trajectory and smooth attractors for Cahn-Hilliard equations with inertial term. Nonlin. 23 (2010) 707-737.

[28] F. Hecht, New development in FreeFem++. J. Numer. Math. 20 (2012) 251-265.

[29] Z. Hu, S. Wise, C. Wang and J. Lowengrub, Stable and efficient finite-difference nonlinear-multigrid schemes for the phase-field crystal equation. J. Comput. Phys. 228 (2009) 5323-5339.

[30] S.-Z. Huang and P. Takáč, Convergence in gradient-like systems which are asymptotically autonomous and analytic. Nonlin. Anal. 46 (2001) 675-698.

[31] J.-L. Lions, Quelques méthodes de résolution des problèmes aux limites non linéaires. Dunod, Gauthier-Villars, Paris (1969).

[32] S. Łojasiewicz, Ensembles semi-analytiques. I.H.E.S Lecture note, Ecole Polytechnique, Paris (1965).

[33] B. Merlet and M. Pierre, Convergence to equilibrium for the backward Euler scheme and applications. Commun. Pure Appl. Anal. 9 (2010) 685-702.

[34] H. Ohnogi and Y. Shiwa, Instability of spatially periodic patterns due to a zero mode in the phase-field crystal equation. Physica D. 237 (2008) 3046-3052.

[35] M. Pierre and A. Rougirel, Stationary solutions to phase field crystal equations. Math. Methods Appl. Sci. 34 (2011) $278-308$.

[36] N. Provatas, J.A. Dantzig, B. Athreya, P. Chan, P. Stefanovic, N. Goldenfeld and K.R. Elder, Using the phase-field crystal method in the multi-scale modeling of microstructure evolution. J. Miner. Metals Mater. Soc. 59 (2007) 83-90.

[37] J. Simon, Compact sets in the space $L^{p}(0, T ; B)$. Ann. Mat. Pura Appl. 146 (1987) 65-96.

[38] P. Stefanovic, M. Haataja and N. Provatas, Phase-field crystals with elastic interactions. Phys. Rev. Lett. 96 (2006) 225504.

[39] P. Stefanovic, M. Haataja and N. Provatas, Phase-field crystal study of deformation and plasticity in nanocrystalline materials. Phys. Rev. E 80 (2009) 046107.

[40] J. Swift and P.C. Hohenberg, Hydrodynamic fluctuations at the convective instability. Phys. Rev. A 15 (1977) $317-328$.

[41] C. Wang and S.M. Wise, Global smooth solutions of the three-dimensional modified phase field crystal equation. Methods Appl. Anal. 17 (2010) 191-211.

[42] C. Wang and S.M. Wise, An energy stable and convergent finite-difference scheme for the modified phase field crystal equation. SIAM J. Numer. Anal. 49 (2011) 945-969.

[43] S. Wise, C. Wang and J. Lowengrub, An energy-stable and convergent finite-difference scheme for the phase field crystal equation. SIAM J. Numer. Anal. 47 (2009) 2269-2288.

[44] X. Wu, G.J. van Zwieten and K.G. van der Zee, Stabilized second-order convex splitting schemes for Cahn-Hilliard models with application to diffuse-interface tumor-growth models. Int. J. Numer. Methods Biomed. Eng. 30 (2014) 180-203. 